\title{
7 Positionieren mit lokaler Reichweite: Formen und Funktionen epistemischer und evaluativer Positionierungspraktiken zur Vergemeinschaftung und Distinktion
}

In diesem Kapitel werden basierend auf im Laufe des Analyseprozesses gebildeten Kollektionen (vgl. Abschnitt 5.2) Praktiken beschrieben, mit denen sich die Teilnehmenden in Bezug auf konkrete Serien als Positionierungsobjekt (vgl. zur Definition Abschnitt 6.5.1) epistemisch und evaluativ positionieren, d.h. die rekonstruierten kommunikativen Aufgaben bearbeiten (Abschnitt 6.3). Damit wird Positionierung auf lokaler Ebene, also innerhalb der jeweiligen Interaktionssituation betrachtet. Für die Analyse werden folgende Bedingungen berücksichtigt: In Abschnitt 6.2 wurde herausgearbeitet, dass diese Praktiken vor dem Hintergrund der interaktiven Ziele Partizipation und Vergemeinschaftung und Distinktion kontextualisiert sind. Weiterhin ist im Hinblick auf konkrete Serien die Variabilität des common grounds bedeutsam. Denn Interagierende können eine Serie noch nie gesehen haben, nur einzelne Episoden kennen, mehrere Staffeln oder die ganze Serie (vielleicht sogar mehrfach) geschaut haben, sie können über Hintergrund-Informationen verfügen und individuelle Bedeutungen bei der Rezeption konstruieren. Daher besteht eine der kommunikativen Aufgaben darin, Wissen anzuzeigen. Serien als ästhetische Gegenstände erfordern es zudem, eine evaluative Haltung gegenüber ihnen zu kommunizieren, d.h. die andere Aufgabe ist es, zu bewerten. Für das epistemische Recht auf Evaluation ist es notwendig, dass über die epistemische Haltung ein ausreichender epistemischer Status kommuniziert wird (vgl. dafür und für die folgenden Begriffe und Zusammenhänge auch Abschnitt 4.3).

Heritages Terminologie folgend wird dabei zwischen einem $\mathrm{K}^{+}$- und einem $K^{-}$-Status unterschieden: $K^{+}$bedeutet, dass eine Person über ihre epistemische Haltung Wissen in Bezug auf eine Serie anzeigt (Abschnitt 7.1), während $K^{-}$bedeutet, dass die Person über die epistemische Haltung wenig oder gar kein Wissen in Bezug auf eine Serie kommuniziert (Abschnitt 7.2). Der epistemische Status ( $K^{-}$bis $K^{+}$) wird für diese Studie allerdings nicht binär und einander ausschließend - d.h. eine Person ist entweder $\mathrm{K}^{-}$oder $\mathrm{K}^{+}$- gedacht, sondern als Kontinuum modelliert (Abschnitt 6.4). Angelehnt an das interaktionstheoretisch begründete Verständnis von Positionierungen (Abschnitt 3.3) kann die epistemische Positionierung flexibel und dynamisch sein. Daher ist es möglich, den epistemischen Status im Laufe einer Sequenz hoch- oder herunterzustufen.

Für die Partizipation an der Interaktion ist zunächst ein ausreichender epistemischer Zugang zu dem Positionierungsobjekt notwendig, d.h. es wird angezeigt, 
dass möglichst viel von der diskutierten Serie - präferiert über eigene Erfahrungen, also die eigene Rezeption der Serie - bekannt ist. Die epistemische Haltung gibt dabei sowohl Aufschluss über die Gewissheit als auch über die Glaubwürdigkeit des epistemischen Status. Zudem können Teilnehmende über das Recipient Design markieren, inwiefern sie die unterschiedlichen epistemischen Status der anderen Beteiligten adressieren. Insgesamt können sie so anzeigen, wie hoch der eigene epistemische Status ist. Wenn im Laufe der Sequenz die epistemische Haltung eine höhere epistemische Gewissheit und höhere Glaubwürdigkeit der Wissensquelle signalisiert, kann der epistemische Status hochgestuft werden; umgekehrt kann natürlich über geringere Gewissheit und Glaubwürdigkeit der Quelle der Status auch heruntergestuft werden. Auf diesem Wege können Interagierende epistemische Autorität für ihre Bewertung beanspruchen; d.h. sie zeigen, dass sie nicht nur mehr wissen als die anderen, sondern es auch besser oder anders wissen. Sie können zeigen, dass sie über Wissensbestände verfügen, die beispielsweise auf Hintergrund- und Kontextinformationen verweisen, die medienbezogene Fachterminologie oder Kenntnis über narrative Strukturen anzeigen.

Das Kapitel ist folgendermaßen strukturiert: In Abschnitt 7.1 wird analysiert, über welche Positionierungspraktiken Teilnehmende mit einer angezeigten $\mathrm{K}^{+}$Position ihren epistemischen Status so hochstufen, dass sie epistemische Autorität beanspruchen, während in Abschnitt 7.2 herausgearbeitet wird, wie Personen aus einer $K^{-}$-Position ihren epistemischen Status hochstufen. Anschließend wird der Fokus erweitert und nicht mehr nur einzelne Sprechende, sondern die ganze Gruppe in den Blick genommen. Dafür werden Sequenzen in Form von Fallstudien analysiert, in denen im Laufe des Interaktionsprozesses über geteiltes Wissen und Bewertungen sowie das Anpassen und Verschieben der Haltungen Gemeinschaft und Distinktion dynamisch hergestellt werden (Abschnitt 7.3). Die Praktiken werden zur Veranschaulichung in das in Abschnitt 6.4 eingeführte Koordinatensystem schematisch integriert sowie mit Blick auf folgende Aspekte beschrieben und diskutiert:

- Strukturstelle(n) - Welche interaktiven Kontexte können als Ausgangslage zur Durchführung der Praktik gegeben sein?

- Ausgestaltung - Welche Realisierungsformen kann die Praktik annehmen? Auf welche Ressourcen kann dabei zurückgegriffen werden?

- Interaktive Funktion(en) - Für welche Ziele kann die Praktik eingesetzt werden? 


\subsection{Positionierungspraktiken zum Hochstufen des epistemischen Status bei $K^{+}$}

In diesem Abschnitt werden vier Praktiken beschrieben, mit denen Interagierende, die im Laufe der Sequenz eine $\mathrm{K}^{+}$-Position signalisieren, ihren epistemischen Status höherstufen und damit epistemische Autorität beanspruchen bzw. interaktiv aufrechterhalten können:

(1) Managen divergenter Wissens(be)stände

(2) Einbeziehen serienexterner Wissensquellen

(3) Absprechen von fremder epistemischer Autorität zur Bewertung einer Serie

(4) Empfehlen eines Rezeptionsmodus

$\mathrm{Zu}$ jeder Praktik werden meistens drei Fallbeispiele aus der entsprechenden Kollektion analysiert, anhand derer jeweils verschiedene interaktive Realisierungsformen der Praktik sowie der Einsatz verschiedener Ressourcen erläutert werden. Zur Veranschaulichung sind die Unterkapitel zunächst nach dem Positionierungsobjekt - dem Titel der verhandelten Serie - und dann nach der Ausrichtung der Praktik benannt. Die Abschnitt sind dabei jeweils folgendermaßen strukturiert: Nach einer kurzen Synopse des Inhalts der Sequenz und ihrer kontextuellen Einbettung erfolgt eine auf die Beschreibung und Interpretation der Praktik zugeschnittene Analyse des Falls unter Beantwortung einer spezifischen analyseleitenden Fragestellung. Jedes Abschnitt endet mit einer Zusammenfassung und Diskussion der Praktik entlang der Aspekte Strukturstelle(n), ihre interaktive Ausgestaltung sowie Funktionalisierung.

\subsubsection{Managen divergenter Wissens(be)stände}

Eine besondere Herausforderung insbesondere in Mehrparteien-Interaktion liegt in der Gestaltung des Recipient Designs im Hinblick auf den epistemischen Status der anderen Beteiligten. Je nachdem, als wie viel und was wissend sich die ko-präsenten Interagierenden darstellen, müssen die eigenen Äußerungen entsprechend angepasst werden. Beteiligte mit einem hohen epistemischen Status können sich als Personen mit Expertise positionieren, indem sie die individuell verschiedenen Wissensstände und Wissensbestände der Gruppe berücksichtigen.

Speziell das Gesprächsthema Serien erfordert die interaktive Kunst, Wissen so zu teilen, dass drei unterschiedliche Anforderungen gleichermaßen erfüllt werden: So muss so viel Wissen preisgegeben werden, um sich selbst als $\mathrm{K}^{+}$zu positionieren. Eine damit einhergehende interaktive Funktion ist auch die auf geteilten Bewertungen basierende Vergemeinschaftung mit anderen Wissenden. Eine wei- 
tere Funktion - die besonders in dem hier vorliegenden Gesprächstyp verlangt wird - besteht darin, den $\mathrm{K}^{-}$-Beteiligten ausreichend Wissen an die Hand zu geben, damit sie ebenfalls am Gespräch (evaluativ) partizipieren können (Abschnitt 6.2.1). Schließlich dürfen $\mathrm{K}^{-}$-Beteiligte oder $\mathrm{K}^{+}$-Beteiligte, die noch nicht die ganze Serie geschaut haben, auch nicht $z u$ viel Wissen erhalten, da ansonsten die Gefahr des Spoilerns besteht (vgl. Abschnitt 8.2.3).

Dieses von Personen mit Expertise vollzogene Ausbalancieren der verschiedenen Wissensstände wird im Folgenden anhand dreier Realisierungsformen der Praktik veranschaulicht. In Beispiel 13 richten sich die $K^{+}$-Sprecher primär an die $K^{-}$-Beteiligten, während sie in Beispiel 14 sowohl $\mathrm{K}^{-}$- als auch andere $\mathrm{K}^{+}$Positionen gleichzeitig managen. Zwei Fälle (Beispiele 15 und 16) zeigen dagegen Sequenzen, in denen sich die $\mathrm{K}^{+}$-Sprechenden primär an den anderen $\mathrm{K}^{+}$Sprechenden orientieren. Leitend für die Beschreibungen dieser Praktik ist die Frage: Wie managen $\mathrm{K}^{+}$-Beteiligte divergierende Wissensstände und Wissensbestände der Gruppe und welche interaktiven Konsequenzen ergeben sich daraus?

\subsubsection{FARGo - Primäre Orientierung an $\boldsymbol{K}^{-}$}

(13) der HOBbit spielt da mit?

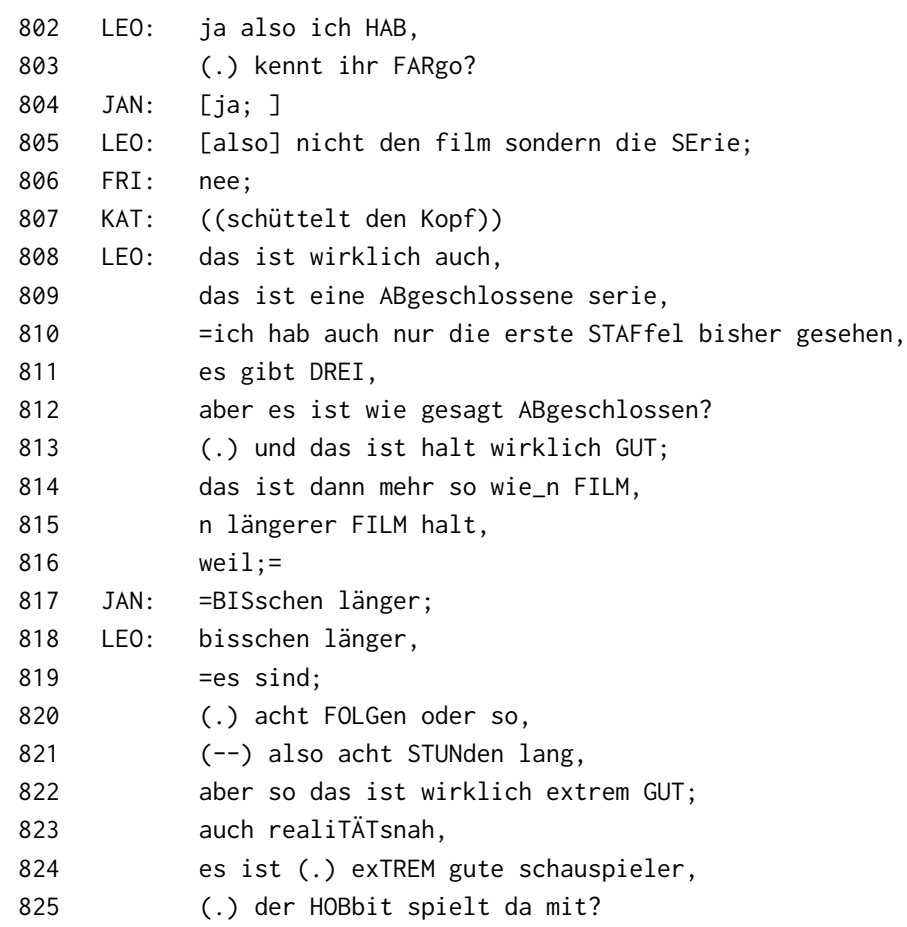




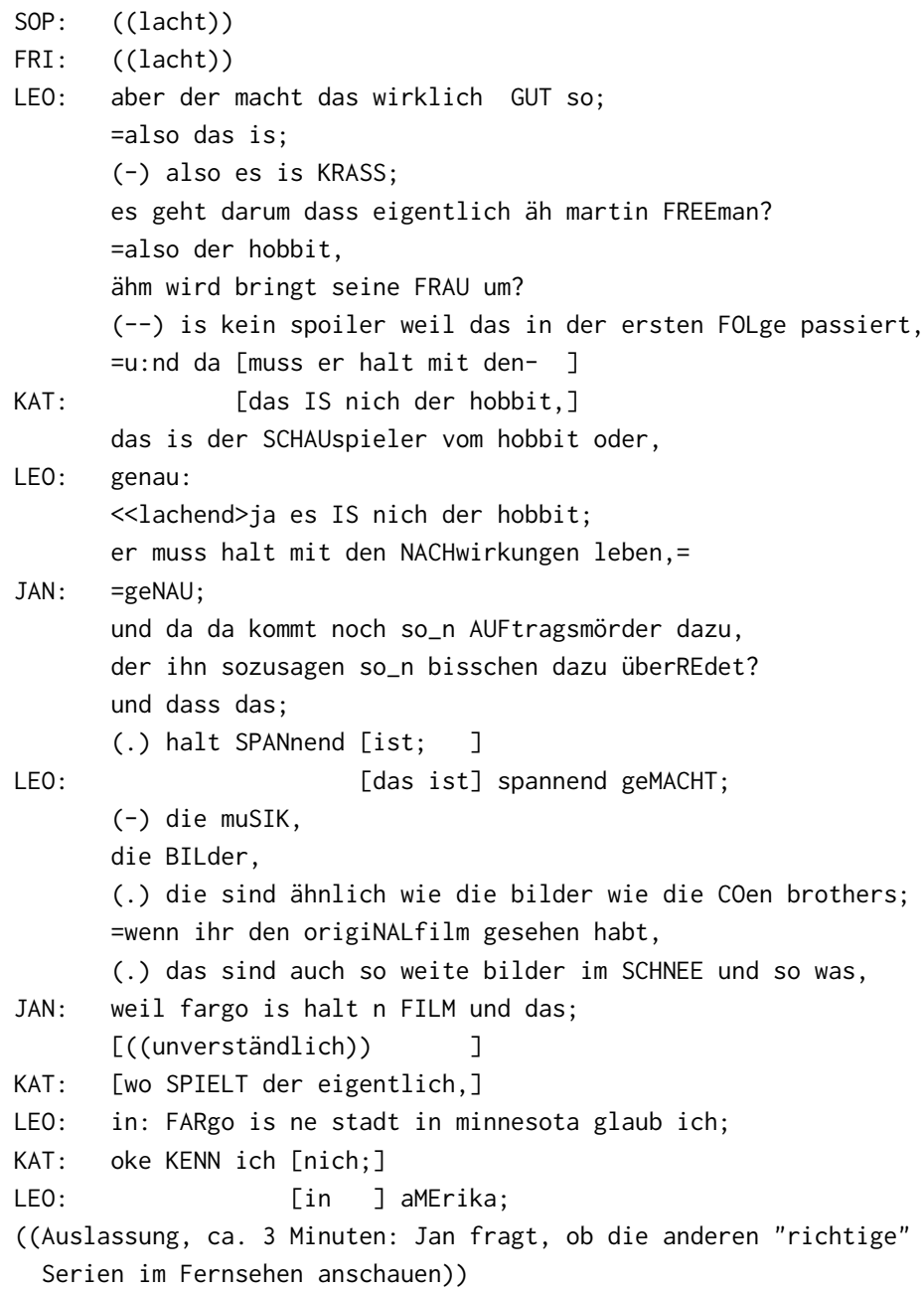

RHG10, 19:22-20:24

Bevor die hier ausgewählte Sequenz beginnt, wurde Leon von der Gruppe als nächster Sprecher ausgewählt, der seine Lieblingsserie nennen soll (vgl. zur Strukturierung der Gespräche Abschnitt 6.1.1). Er führt die Serie FARGO an, zu der sowohl er als auch Jan sich als $K^{+}$positionieren (Z. 802-804), während Friederike und Katharina ihren epistemischen Status explizit als $\mathrm{K}^{-}$markieren (Z. 806-804). Oliver und Sophie äußern während des Sequenzverlaufs keine verbal oder nonverbal erkennbare Positionierung. Leon und Jan fassen daraufhin interaktiv gemeinsam den Plot der Serie zusammen und begründen, warum sie ihnen gefällt (Z. 808-853). 
Katharina stellt währenddessen Verständnis- (Z. 836-837) und Informationsfragen (Z. 854-857).

Bevor Leon die Serie FARGo als Positionierungsobjekt etabliert (Z. 801), erfragt er mittels einer selbstinitiierten Reparatur - er wechselt von der abgebrochenen Initiierung, die erkennbar den vorherigen Zugzwang des Nennens der Lieblingsserie bedient: ,ich hab, “ (Z. 802) zu der Formulierung „kennt ihr FARgo?“ (Z. 802) - den epistemischen Status der anderen. Damit eröffnet er allen Beteiligten die Chance, ihre eigene epistemische und ggf. auch evaluative Haltung zu markieren. Die Teilnehmenden können sich also selbst im ,Positionierungsfeld“ (vgl. Abschnitt 6.4) einordnen, was es Leon erleichtert, seine nachfolgenden Äußerungen den jeweiligen Kenntnisständen und Bewertungen angepasst zu designen. Leon konzentriert sich im Folgenden vorrangig auf den $\mathrm{K}^{-}$-Status von Friederike und Katharina, während er Jans $\mathrm{K}^{+}$-Status nicht explizit adressiert, sondern ihn quasi ,nebenbei' bedient. Deutlich wird das daran, dass Leon die ganze Sequenz über den $K^{-}$-Teilnehmenden körperlich zugewandt bleibt und sich zu keiner Zeit zu dem neben ihm sitzenden Jan dreht - auch nicht, als Jan ihn beispielsweise mittels einer Reparatur direkt adressiert (Z. 816-818).

Um die $K^{-}$-Beteiligten einzubeziehen, schließen Leon und Jan ko-konstruktiv deren Wissenslücken. Sie beanspruchen Expertise, indem sie vielfältige Wissenselemente äußern, die sich auf die Präsentationsform der Serie (Z. 808-809, 813-816, 819-821), die Qualität des Hauptdarstellers (Z. 824-830), den Beginn des Handlungsstrangs (Z. 831-843) mit explizitem ,Spoiler-Disclaimer' (Z. 834; vgl. dazu auch Abschnitt 8.2.3) und schließlich auf die visuelle Ästhetik (Z. 845-851) beziehen. Im Laufe der Sequenz wird deutlich, dass Leon sich zwar als $K^{+}$positioniert, seine Haltung allerdings auch dadurch wieder relativiert, dass er angibt, von drei Staffeln bisher nur die erste rezipiert zu haben (Z. 810). Dieser Aspekt ist jedoch für seine Positionierung als Experte nicht relevant, da er innerhalb der Gruppe relativ gesehen den höchsten epistemischen Status in Bezug auf die Serie beansprucht, der ihm auch weder abgesprochen noch in Frage gestellt wird. Die Informationen, die die $\mathrm{K}^{+}$-Teilnehmer teilen, bewerten beide positiv (Z. 828, 830, 845-846) - positionieren sich also als wissende Unterstützer -, wobei sie potentiell das Feld für zustimmende Zweitbewertungen eröffnen und damit Partizipation ermöglichen. Solche Zweitbewertungen, die aufgrund des $\mathrm{K}^{-}$-Status der Beteiligten i.d.R. keine unabhängige epistemische Autorität beanspruchen und beispielsweise durch Formulierungen wie „das hört sich gut an“ o.Ä. realisiert werden können (vgl. dazu Abschnitt 7.2.2), werden in dieser Sequenz allerdings nicht geäußert.

Um den $\mathrm{K}^{-}$-Status der Adressierten zu managen, verweist Leon auf Anknüpfungspunkte, die er im common ground der Gruppenmitglieder mehr oder weniger sicher verortet. Dazu zählt beispielsweise die Figur, die der Hauptdarsteller Martin Freeman in der Trilogie DeR HobBit (Z. 825, 831-832) spielt, oder die Namen 
der sowohl an dem Film FARGo als auch an der gleichnamigen Serie beteiligten Regisseure Ethan und Joel Coen (Z. 849-850). Seine Kategorisierung der Serie als „realitätsnah“ (Z. 823) lässt eine Orientierung an Friederike als Adressatin erkennen, die zuvor die Serie THE WIRE wegen ihres Realitätsbezugs als positiv bewertet hat (vgl. Beispiel 19). Katharina stützt Leons Partizipationsbestrebungen, indem sie seine Formulierung zur Entfaltung der Handlung (,der hobbit, ähm wird bringt seine FRAU um? “, Z. 832-833) - die einen scheinbaren intertextuellen Zusammenhang nahelegt - scherzhaft aufgreift (Z. 836-837) und eine Informationsfrage nach dem Setting der Serie stellt (Z. 854).

Leon managt parallel dazu auch Jans hohen epistemischen Status, indem er ihn als „Co-Teller“ (Lerner 1992) ratifiziert und ihn beim Rekonstruieren assistieren lässt. So kann Jan an Leons Turns fast nahtlos anschließen und ihn fortführen (Z. 840-841, 845-846, 851-852) sowie zustimmende Bewertungen, die unabhängige epistemische Autorität beanspruchen (Z. 845), äußern. Potentiell wäre noch Raum für entweder Nachfragen der $K^{-}$-Beteiligten oder weitere Rekonstruktionen und Bewertungen der $\mathrm{K}^{+}$-Teilnehmer, allerdings wird die Sequenz anschließend durch Jans Etablierung eines neuen Positionierungsobjekts (Z. 858) beendet.

Die Anforderung bei der epistemischen Positionierung gegenüber $\mathrm{K}^{-}$- Teilnehmenden in Bezug auf Serien wird hier erkennbar: $K^{+}$-Teilnehmende müssen solche Wissenselemente über die Serie äußern, die an (angenommenes) geteiltes Wissen anschließen, um die Gelegenheit für Äußerungen, Nachfragen, Bewertungen usw. zu geben und damit (auch) das Ziel der gemeinsamen, diskursiven Bearbeitung der von der Forscherin gestellten Aufgabe zu erfüllen. Gleichzeitig dürfen sie aber nicht zu viele Informationen über die Handlung selbst preisgeben (vgl. Z. 834), da ansonsten der Reiz für die $K^{-}$-Beteiligten, die Serie möglicherweise in Zukunft selbst anzuschauen, verloren geht. Aus diesem Grund bewerten Leon und Jan die Serie aufgrund einer Vielzahl von Aspekten als positiv, die sich kaum auf die konkrete Handlungsebene beziehen, und markieren die handlungsbezogenen Informationen als keine Spoiler. Die interaktive Funktion des Ermöglichens von Partizipation steht hier etwas mehr im Vordergrund als die Vergemeinschaftung zwischen Jan und Leon über die gemeinsame Bewertung der Serie.

\subsubsection{THE 100 - Orientierung sowohl an $K^{-}$als auch an $K^{+}$ (14) du hast the HUNdred angesprochen;=ne,}

\footnotetext{
555 NEL: [is_n film]

556 LEN: du hast jetzt [the hund,]

$557 \quad(-)$ du hast the HUNdred angesprochen;=ne,

558 ich wollt das angucken wegen sharMA;

$559=$ =ihr wisst ja sharma ist mein MANN,
} 
ALLE: $\quad[(($ lachen $))$

LEN: [er hat ja bei EIner folge zwei] in der dritten sch:taffel glaub ich,

(.) äh w worum geht,

=ich weiß nur [dass da einhundert MENschen sind?]

EMI : [ (um hundert und ALso;)

also die sind, [ähm ]

JOS: [geht_s] nich um TIEre?

MAG: 'HM' hm;

JOS: ach [nein das ist the Z00; ]=ne,

EMI : [(oh mein GOTT; ) ]

MAG: $\mathrm{mHM}$,

ALLE : [((lachen $)) \quad]$

EMI: also,

(-) also GUCK mal;

das.

$(-)$ die Erde ist halt sozusagen verLASSen?

weil (.) irgendwie GING das da nich mehr von den

[lEbensverhältnissen] [und dann sind-]

MAG: [naTURkatastrophe; ]

LEN:

EMI :

[ach so; ]

[ja; ]

(.) dann sind die halt im (.) s WELTall?

(.) und irgendwann geht diese station aber irgendwie kaPUTT und die müssen,

(.) halt DIE so;

das sind so hundert geFANGene,

so jugendliche die irgendwas gemAcht haben,

$(-) \quad[$ und $(x x x)]$

LEN: [auf ] der ERde,

MAG: 'HM' hm;

EMI: nein [im ] (.) WELTall;

LEN: $\quad$ [ach SO;]

EMI: dann schicken die die halt RUNter um zu gucken ob die überleben,

weil eGAL is ob die sterben?

(.) .h ähm und dann überLEgen, GuCken die halt so wie du die-

das da [so die lebensverhältnisse]

JOS:

[töten die sich

] GEgenseitig,

oder warum heißt das [the ] HUNDdred;

EMI : $\quad$ [nee;]

nee das sind einfach hundert (.) !TEEN!ager;

JOS: aber da sterben TROTZdem welche;=[ne,]

$M A G$ :

$[\mathrm{JA}],[\mathrm{ja}$.

EMI :

$[j a ;]$

MAG: weil da da irgendWIE_äh; 


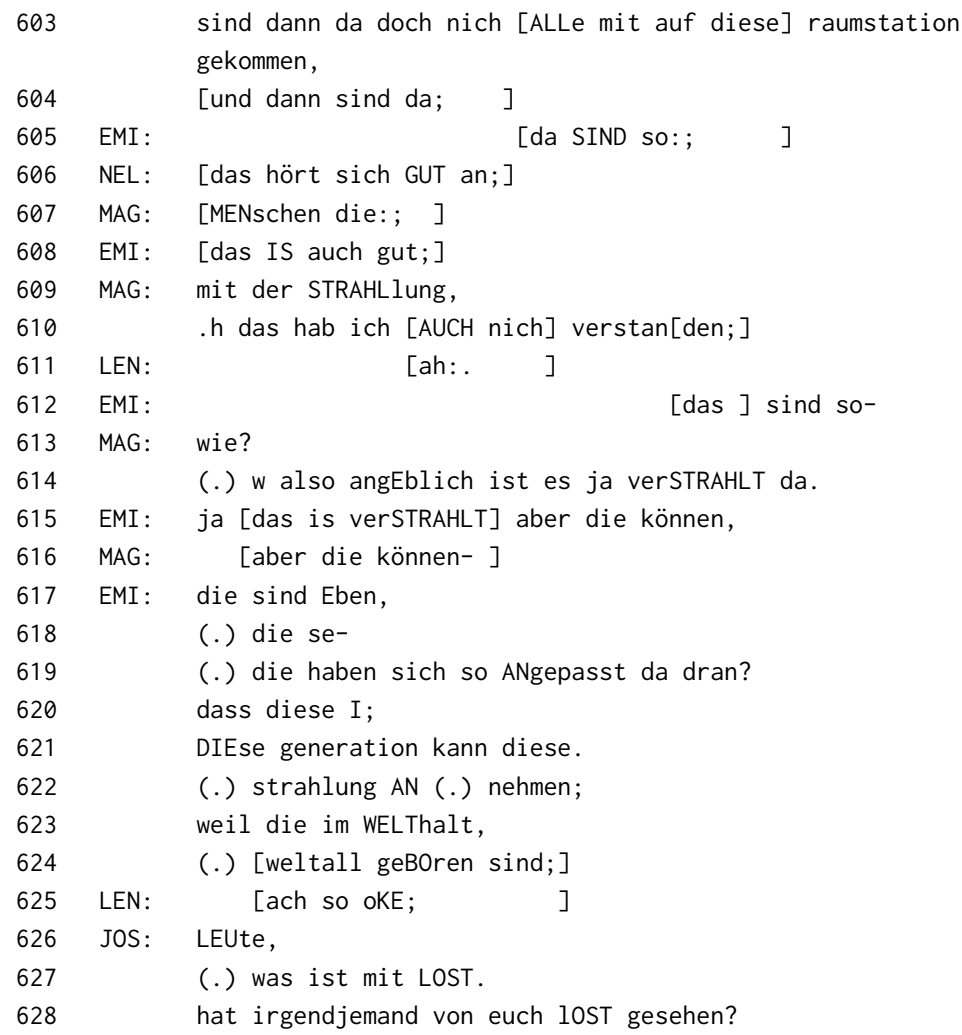

Die Sequenz beginnt, indem Leni Emilia adressiert und sie fragt, worum es in der Serie THE 100 geht. Daraufhin positionieren sich Emilia, Magnus und Martha als $K^{+}$, während Leni, Nelli und Josefine sich als $K^{-}$(Z. 562-563; 606; 566-569) positionieren. Bilel kommuniziert seinen epistemischen Status nicht. Im Laufe der Sequenz fasst Emilia unter Nachfragen und Ergänzungen der anderen Gruppenmitglieder Informationen über die Figuren und Schauplätze der Serie zusammen (Z. 572-608) und diskutiert anschließend mit Magnus Fragen der Handlungslogik (Z. 609-625), bevor Josefine mit der Thematisierung von Lost die Sequenz beendet (Z. 626-628).

Emilia agiert hier als diejenige, die unterschiedliche Wissens(be)stände managt sowie Wissensasymmetrien ausgleicht und sich damit als Expertise beanspruchend positioniert. Diese Rolle wird ihr interaktiv durch Lenis Fremdpositionierung (Z. 562-563), aber auch durch Magnus' Interaktionsverhalten (Z. 609-610, 613614) zugeschrieben und anerkannt. Auch sie orientiert sich - ähnlich wie Leon 
in Beispiel 13 - zunächst an Leni und ihrer $K^{-}$-Position. Bevor sie von Josefine unterbrochen wird (Z. 566), formuliert sie einen Turn, der strukturell an Lenis minimales Wissen anzeigende Äußerung, es ginge um „einhundert MENschen“ (Z. 563), anschließt: „,also die sind,“ (Z. 565). Dann startet sie nach einer aufmerksamkeitssichernden Äußerung (Z. 573) eine Zusammenfassung der Ausgangssituation der Handlung, während sie mit ihrem Blick Leni adressiert. Ihre Äußerungen sind von vagen Modalisierungen durchzogen: ,halt“ (Z. 575, 580), ,irgendwie“ (Z. 576, 581), „irgendwann“ (Z. 581), „So“ (Z. 582, 584), die Leni allerdings nur punktuell durch Nachfragen (Z. 586) relevant setzt. Ansonsten ratifiziert sie Emilias Wissensübermittlung mit Erkenntnisprozessmarkern (zum Begriff vgl. Abschnitt 4.3) (Z. 578, 589, 611, 625).

Gleichzeitig positioniert Emilia sich selbst - und wird auch so fremdpositioniert - ebenfalls als verantwortlich für den Umgang mit Josefines und Magnus' Wissensäußerungen. Josefine zeigt mehrfach minimales und sich im Nachhinein als nicht korrekt erweisendes Wissen an (Z. 566, 595-596, 599, vgl. auch Abschnitt 7.1.2), signalisiert diesbezüglich aber meistens eine relativ hohe epistemische Gewissheit. Emilias Reaktion auf Josefines Selbstreparatur bezüglich der Verwechslung mit der Serie THE Zoo („oh mein GOTT; “, Z. 569) sowie die Zurückweisung ihres Erklärungsversuchs für den Titel der Serie (Z. 595-596) fremdpositioniert sie eindeutig als $\mathrm{K}^{-}$und signalisiert ihr damit, dass sie keine epistemische Autorität in Bezug auf diese Serie beanspruchen kann (vgl. auch Abschnitt 7.1.3). Lediglich ihre abschließende Wissenskommunikation ,aber da sterben TROTZdem welche;=ne, “ (Z. 599) ratifizieren sowohl Emilia als auch Magnus. Magnus beansprucht - vergleichbar mit Jan in Beispiel 13 - ebenfalls durch Reparaturen, die Emilias vage Aussagen konkretisieren („naTURkatastrophe“, Z. 577), sowie durch seine Beurteilung und Umgang mit Josefines epistemischer Haltung (Z. 602-604) eine $K^{+}$-Position für sich. Zugleich konstruiert er diese Position als Emilias untergeordnet, indem er sie mit einer Verständnisschwierigkeit bezüglich der Handlungslogik adressiert (Z. 609-616). Die Adressierung wird dadurch deutlich, dass er indexikalisch so voraussetzungsreich formuliert, dass tieferes Wissen über die Vorkommnisse in der Serie notwendig ist, um die Frage beantworten zu können. Emilia bearbeitet auch diese Wissensasymmetrie und jongliert dabei zwei divergierende Wissensstände. Einerseits orientiert sie sich im Rahmen ihrer Erklärung primär an Magnus' vorhandenem Wissen (Z. 619-622, 624). Andererseits adressiert sie implizit auch mit ihrer Blickbewegung Leni, indem sie ihr weitere relevante Informationen den Plot betreffend liefert, was Leni wiederum auch als Wissensausgleich ratifiziert (,ach so“, Z. 625).

Auch wenn Emilias Äußerungen mit Reparaturen, Pausen und vagen Modalisierungen durchzogen sind, erfüllen sie doch im Sinne des Wissensmanagements mehrerer Wissens(be)stände ihren Zweck: Sowohl Lenis als auch Magnus' Wissens- 
lücken werden behoben und auf Josefines ,Halbwissen' reagiert sie entsprechend humorvoll-bewertend. Auch daran zeigt sich die Gratwanderung, genug, aber nicht $z u$ viel Wissen für $\mathrm{K}^{+}$- und $\mathrm{K}^{-}$-Gesprächsbeteiligte anzupassen.

\subsubsection{THE ORIginals und HORSELAND - primäre Orientierung an $\boldsymbol{K}^{+}$}

Die Analyse der folgenden zwei Sequenzen zeigt, wie $K^{+}$-Beteiligte sich in ihrer Wissenskommunikation vor allem an andere Interagierende mit hohem epistemischen Status wenden, um sich mit ihnen evaluativ zu vergemeinschaften. Dadurch können $K^{-}$-Beteiligte meistens kaum oder gar nicht an dem Gespräch partizipieren. Die beiden Fälle zeigen jeweils einen anderen interaktiven Umgang mit dieser Gegebenheit auf. Während in Beispiel 15 eine andere Sprecherin die Rolle der Wissens(be)ständemanagerin übernimmt, wird in Beispiel 16 die Wissensasymmetrie erst im Nachhinein - zumindest geringfügig - behoben.

(15) er HAT sie nicht geküsst;

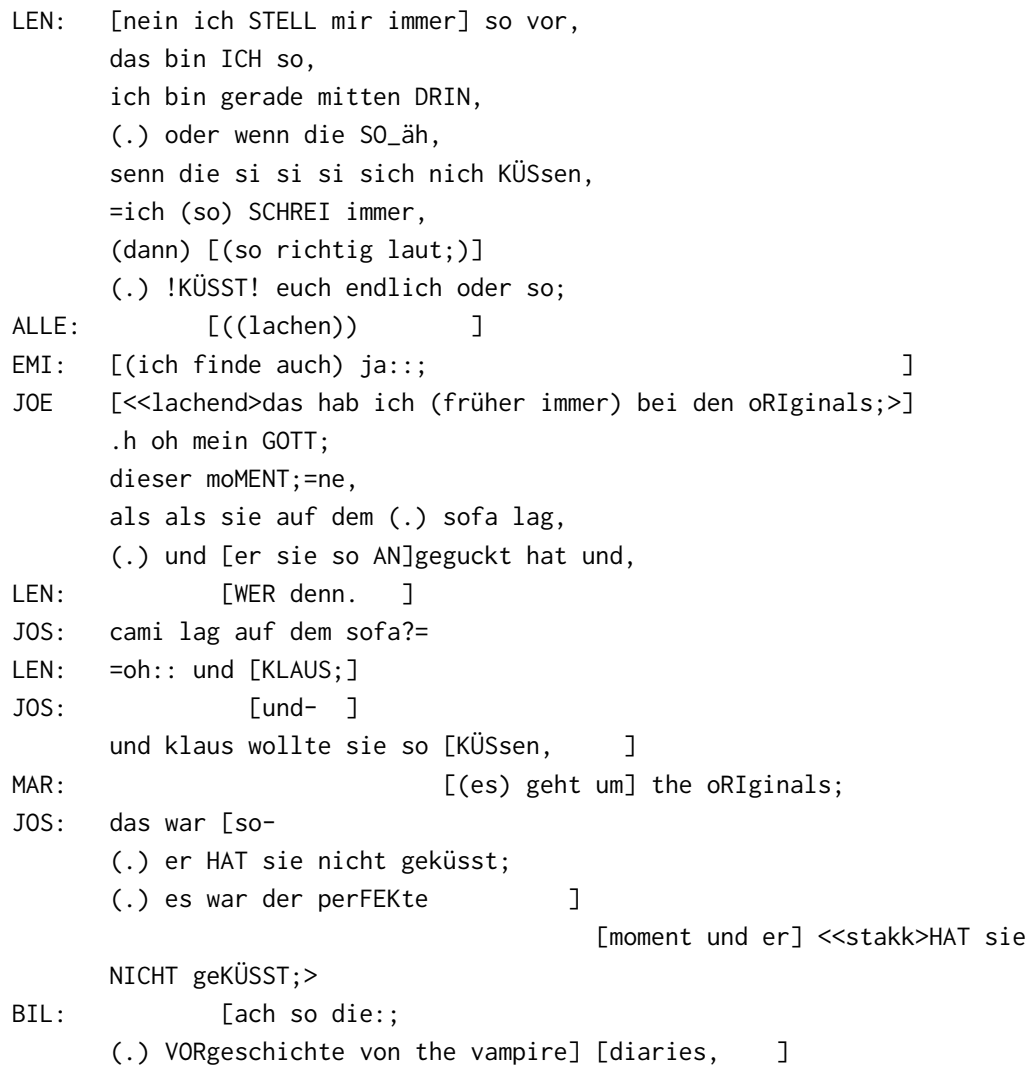




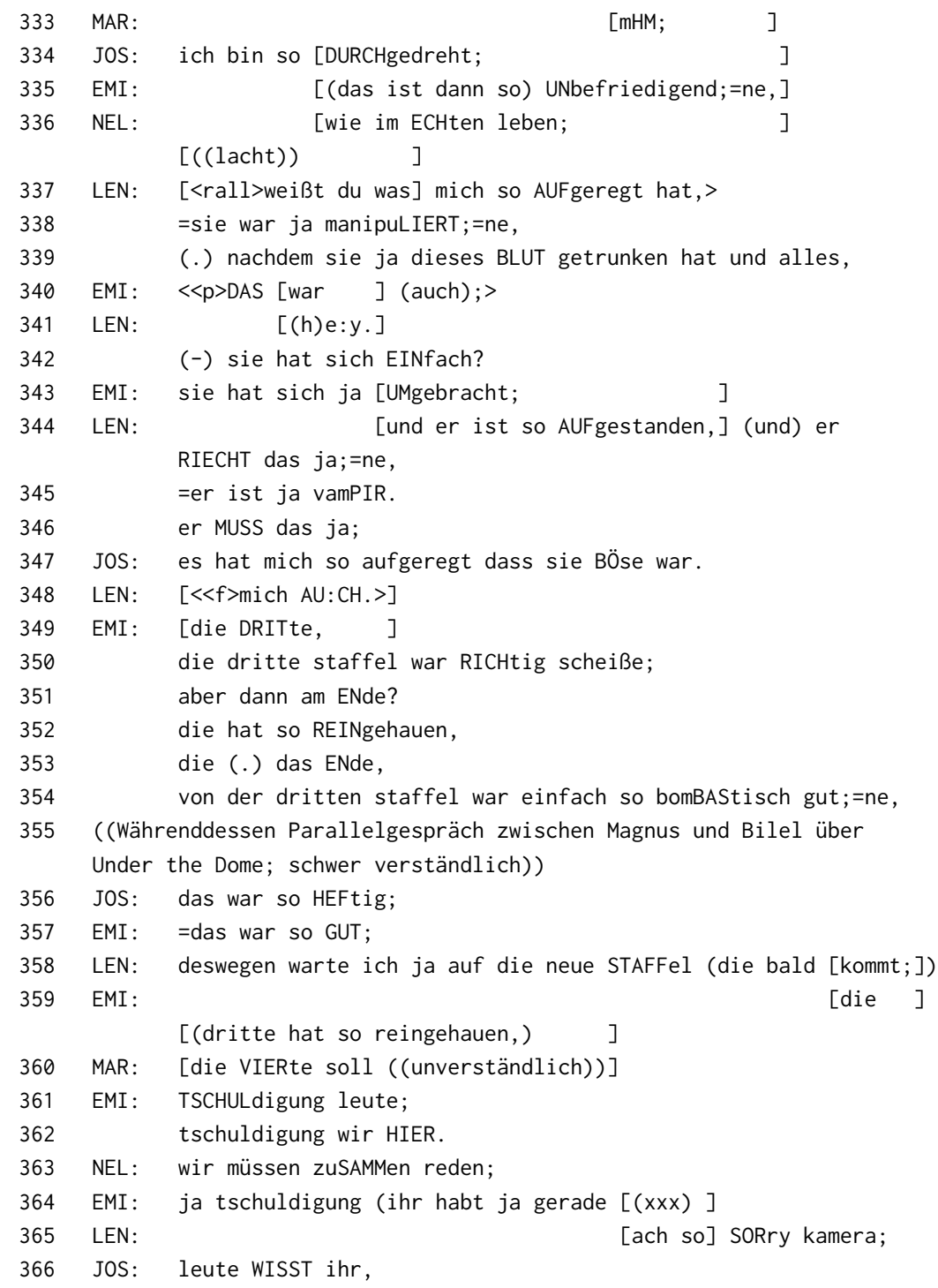

Diese Sequenz schließt an die Aushandlung der von Leni initiierten Frage an, ob die anderen sich genau wie sie mit Serienfiguren identifizieren würden (vgl. dazu auch Abschnitt 8.1.4). In Anlehnung an die anschauliche Rekonstruktion ihres Rezeptionsverhaltens (Z. 307-314) positioniert sich Josefine in Alignment mit ihr in Bezug auf die Serie THE ORIGINALs ebenfalls als $K^{+}$(Z. 317-334). Daraufhin 
entfaltet sich zwischen ihr und Leni - sowie partiell auch mit Emilia - eine zustimmende Bewertungsinteraktion (Z. 324-360). Während des Bewertungsaustauschs signalisieren Bilel und Magnus nonverbal mittels fragender Blicke implizit einen $\mathrm{K}^{-}$-Status, woraufhin Martha ihnen den Namen der Serie mitteilt (Z. 327) und sich die beiden dann parallel einer anderen Serie zuwenden (Z. 355). Die Sequenz wird durch die Aufforderung, wieder als große Gruppe gemeinsam zu interagieren (Z. 361-365) und Josefines Etablierung eines neuen Positionierungsobjekts (Z. 366) beendet.

Das Management der unterschiedlichen Wissensstände übernimmt hier nicht eine Person wie in Beispiel 13 und Beispiel 14, sondern verteilt sich auf mehrere Interagierende. Josefine und Leni vergemeinschaften sich über ihre gemeinsame Kenntnis und Bewertung einzelner Szenen der Serie. Dafür bedienen sie sich überwiegend voraussetzungsreicher, indexikalisch vager Formulierungen (Z. 338, 347) - die nur gelegentlich präzisiert werden müssen (Z. 322) -, um geteiltes Wissen zu aktualisieren (Z. 324) sowie zustimmende Bewertungen hervorzurufen (Z. 347348). Ihre wechselseitig anerkannte Expertise ist die Voraussetzung dafür, dass sie sich eines als ähnlich empfundenen emotionalen Rezeptionserlebens (,ich bin so DURCHgedreht;“, Z. 334 und „weißt du was mich so AUFgeregt hat,“, Z. 337) vergewissern. Sie nutzen jedoch anders als in den zuvor analysierten Beispielen ihre $\mathrm{K}^{+}$-Position nicht, um Wissensasymmetrien auszugleichen. Diese Rolle übernimmt stattdessen Martha, die im Rahmen einer Nebensequenz an Bilel und Magnus adressiert den notwendigen Kontext für das Verständnis dieser Sequenz liefert sowie deren Verstehensprozessmarkierung ratifiziert (Z. 327, 331-333). Damit signalisiert sie ebenfalls einen $\mathrm{K}^{+}$-Status in Bezug auf die Serie. Auch Emilia impliziert einen hohen epistemischen Status, indem sie sich an dem Bewertungsaustausch zwischen Leni und Josefine (Z. 335, 357) beteiligt. In die Rekonstruktionen des emotionalen Erlebens steigt sie dabei jedoch nur punktuell und distanzierter ein (Z. 335, 340, 343) und schließlich verschiebt sie das Gesprächsthema auf die abstraktere Ebene der Bewertung einzelner Staffeln der Serie (Z. 350-360).

Die emotionale Erlebnisqualität spielt für die Positionierung von Leni und Josefine eine zentrale Rolle (vgl. auch Abschnitt 8.2.4.1). So bereitet Josefine beispielsweise den Höhepunkt ihrer Erzählung (Z. 317-330) sorgfältig vor, formt ihn insbesondere prosodisch sehr genau aus (Z. 329-330) und erhält an passender Stelle affiliative Reaktionen von Leni (Z. 324). Damit gestalten beide ihre epistemischen Positionierungen als Expertinnen für die Serie vorrangig für einander als ,Insider‘. Vermutlich ergibt sich auch dadurch bedingt eine Nebenkommunikation der $\mathrm{K}^{-}$-Beteiligten zu einer anderen Serie, nämlich UNDER THE DomE (Z. 355, vgl. auch Abschnitt 7.2.3). Die Instruktion der Forscherin, gemeinsam über Serien zu sprechen (Abschnitt 6.1), führt schließlich indirekt dazu, dass diese Interaktionskonstellation aufgelöst wird (Z. 361-363; vgl. dazu auch die Analyse 
von Beispiel 40 in Abschnitt 6.1.2). Daran wird deutlich, dass in dem in dieser Studie analysierten Gesprächstyp eine derartige, andere eher exkludierende Vergemeinschaftung strukturell erschwert wird bzw. bei ihrem Auftauchen sanktioniert wird. Stattdessen stellt das Managen verschiedener Wissensbestände im Sinne der gleichzeitigen Orientierung an $\mathrm{K}^{+}$- und $\mathrm{K}^{-}$-Positionen für diesen Gesprächstyp eine erfolgreichere Praktik dar. Das zeigt sich auch in etwas anderer Form anhand des folgenden Beispiels, einer Gruppe jüngerer Teilnehmerinnen:

(16) horseland is so DUMM,

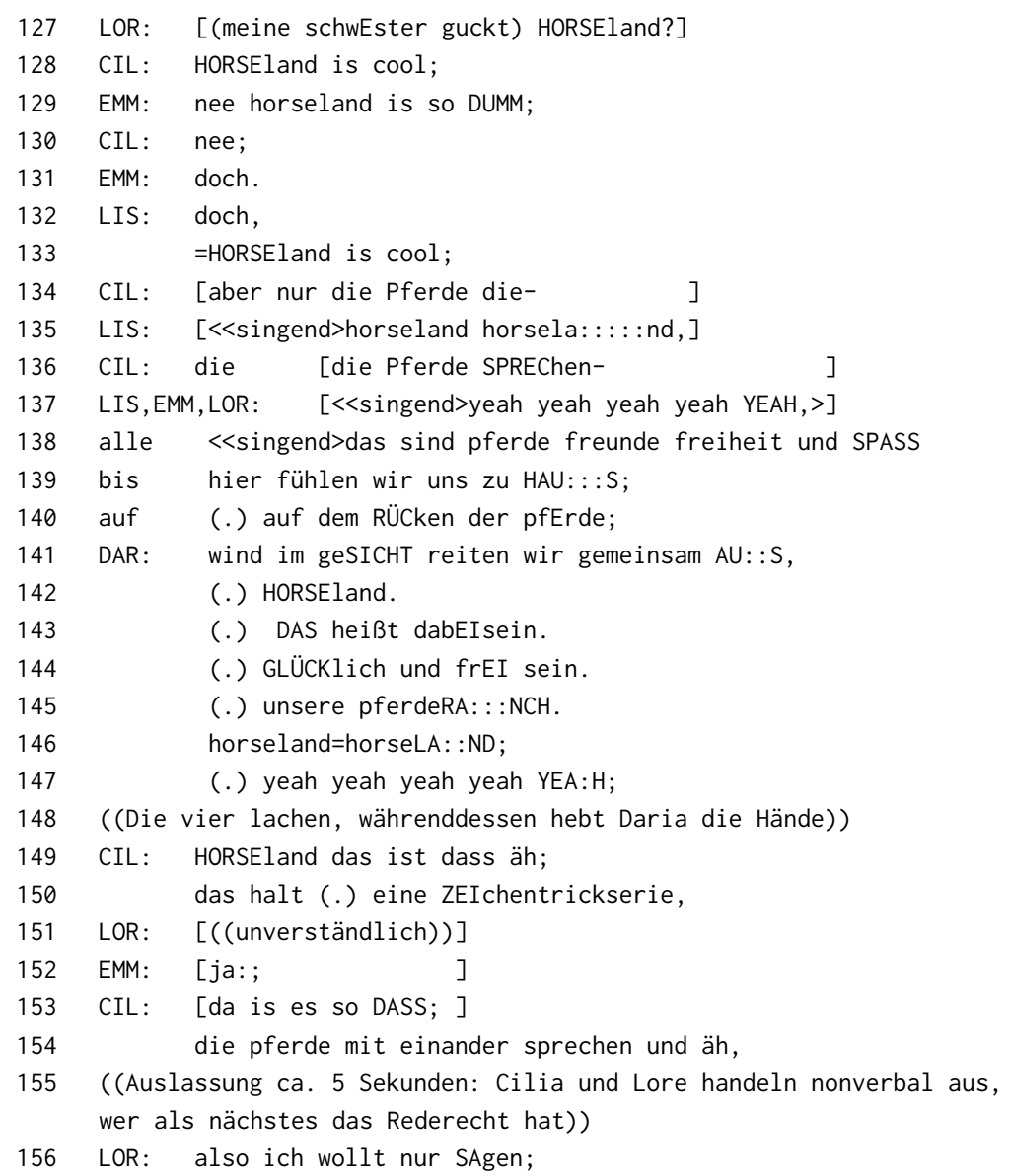


Lore vergleicht die Serie HoRSELAND mit dem zuvor diskutierten Film WENDY und etabliert sie damit als Positionierungsobjekt, das zunächst evaluativ kontrastiv ausgehandelt wird (Z. 127-136). Anschließend singen Cilia, Emmy, Lisa und Lore in die Kamera gewandt das Titellied der Serie (Z. 137-147). Nachdem Cilia einzelne Wissenselemente äußert (Z. 149-154), beenden die Teilnehmerinnen die Sequenz durch gesprächsorganisatorische Aushandlungen (Z. 155). Daria beteiligt sich weder an den evaluativen Aushandlungen noch am Singen des Titelliedes, so dass ihr angezeigter epistemischer Status als $K^{-} \mathrm{zu}$ bezeichnen ist. Ihr für die Partizipation an der Gesangseinlage unzureichendes Wissen signalisiert sie überwiegend nonverbal, indem sie die Hände hebt und mit den Schultern zuckt, während die anderen lachen (Z. 148).

Cilia setzt mit einem Turn offenbar an, Wissen über die Serie zu teilen (Z. 134, 136), wird dann jedoch von Liv, die leise das Titellied singt (Z. 135), unterbrochen. Beide schließen sich der so initiierten Vergemeinschaftungsaktivität an (Z. 137-148). Mit dem Singen des Liedes verweist Lisa auf voraussichtlich im common ground der Gruppe befindliche Wissenselemente, ohne sie als solche explizit zu kennzeichnen (wie es z.B. Leon in Beispiel 13 macht). Bemühungen um den Ausgleich epistemischer Asymmetrie werden hier strukturell an das Ende der Sequenz verschoben. Mit Blicken in die Kamera und an Daria gerichtet erläutert Cilia kurz das Format der Serie als „Zeichentrick“ (Z. 150) und dass es darin um „sprechende Pferde“ (Z. 154) geht. Damit ratifiziert sie erst nachträglich Darias niedrigen epistemischen Status. Zugleich adressiert sie auch durch ihren klar erkennbaren Blick in die Kamera die Forscherin als Nicht-Wissende mit ihrer Erläuterung (vgl. dazu auch Abschnitt 6.1.2). Daria schließt keine Nachfragen an und es werden keine weiteren Wissenselemente verhandelt. Wie in Beispiel 15 steht hier die vergnügliche Vergemeinschaftung über das inszenierende Singen des Liedes im Vordergrund. Bei dieser Sequenz handelt es sich um einen abweichenden Fall, da in den weitaus meisten Fällen des Korpus eine Präferenz für wechselseitiges Bemühen um Partizipation erkennbar ist.

\subsubsection{Zusammenfassung: Die Praktik Managen divergenter Wissens(be)stände}

Die Positionierungspraktik des Managen divergenter Wissens(be)stände wird in Abbildung 7.1 schematisch visualisiert (siehe zur Erläuterung Abschnitt 6.4) und lässt sich folgendermaßen beschreiben:

- Strukturstelle(n): Die Gruppenmitglieder positionieren sich erkennbar sowohl als $\mathrm{K}^{+}$als auch $\mathrm{K}^{-}$in Bezug auf eine konkrete Serie. Eine Person (A) positioniert sich und/oder wird positioniert als diejenige mit der höchsten epistemischen Autorität (eA) zur Bewertung der Serie. 
- Ausgestaltung: Diese Person adressiert $K^{+}$- und $K^{-}$-Beteiligte $\left(\mathrm{B}_{1}\right.$ und $\left.\mathrm{B}_{2}\right)$ durch Verweisen auf Elemente im angenommenen common ground . Als Ressource fungiert dabei vor allem das Rekonstruieren der Handlung, einzelner Szenen oder Rezeptionssituationen. Die Unterschiede in der Adressierung manifestieren sich dabei wie folgt: Der Verweis auf den common ground bei $\mathrm{K}^{+}$-Beteiligten ist implizit, vage und hoch voraussetzungsreich. Der Verweis auf den common ground bei $K^{-}$-Beteiligten ist dagegen explizit und weist stellenweise belehrenden Charakter auf. Zudem erfolgt wechselseitiges Sichern von Verständnis.

- Interaktive Funktion(en): Je nach primärer Orientierung in der Adressierung unterscheidet sich auch die Funktion der Praktik. Bei primärer Adressierung von $\mathrm{K}^{+}$-Beteiligten steht die Vergemeinschaftung über geteilte Bewertungen im Vordergrund. Bei primärer Adressierung von $\mathrm{K}^{-}$-Beteiligten spielen Bewertungen dagegen eine untergeordnete Rolle und der Fokus liegt auf dem Abbau epistemischer Asymmetrie, um Partizipation zu ermöglichen.

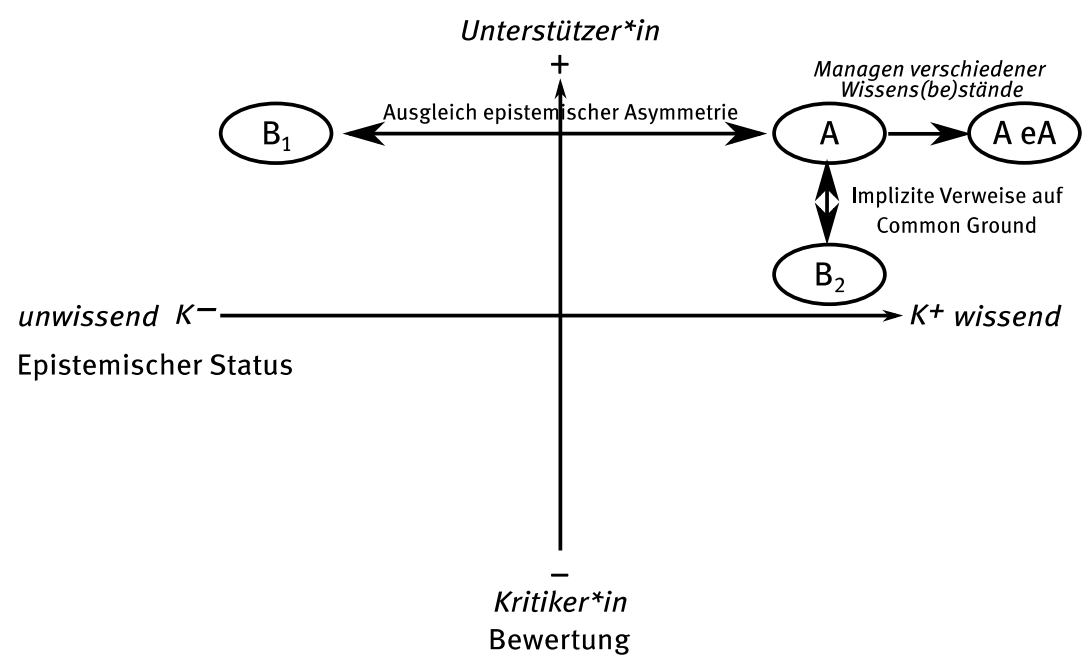

Abb. 7.1: Schematische Darstellung der Praktik Managen divergenter Wissens(be)stände

Das Managen verschiedener Wissensstände und -bestände ist eine komplexe Anforderung. Die Analyse zeigt, dass die ausschließliche Orientierung an $K^{+}$-Beteiligten zu Schwierigkeiten bei der Partizipation und der Aufrechterhaltung der Gruppen- 
diskussion als gemeinsame, diskursive Aufgabe führt. Primär an $K^{+}$-adressierte Positionierungen dienen dazu, evaluative Allianzen innerhalb einer Subgruppe zu formen und sich mit anderen eines geteilten Geschmacks zu vergewissern (Beispiel 15). Diejenigen, die wie Leon (Beispiel 13) oder Emilia (Beispiel 14) ihre Äußerungen so designen, dass sie verschiedene epistemische Status adressieren, sind hinsichtlich der Partizipationsbemühungen der Gruppe erfolgreicher. Die Vereinbarung beider Ziele (Vergemeinschaftung und Partizipationsbestreben, vgl. Abschnitt 6.2) bilden also bisweilen Herausforderungen für die Interaktion.

\subsubsection{Einbeziehen serienexterner Wissensquellen}

Die bisherigen Analysen zeigen, dass Bewertungen zumindest geringen vorhandenen Wissens - in Form eines epistemischen Zugangs - über die Serie bedürfen. Um sich allerdings als Experte oder Expertin zu positionieren, ist es notwendig anzuzeigen, über mehr Wissen als nur die bloße Kenntnis einer Serie zu verfügen. Dafür können $\mathrm{K}^{+}$-Beteiligte auf serienexternes Wissen verweisen, um ihren epistemischen Status hochzustufen. Unter serienexternem Wissen werden Wissenselemente verstanden, die nicht durch die reine Rezeption der Serie erworben werden können, sondern für die weitere, externe Wissensquellen notwendig sind. Die Teilnehmenden können die Glaubwürdigkeit der externen Wissensquelle nutzen, um ihrer eigenen epistemischen Haltung ebenfalls mehr Glaubwürdigkeit $\mathrm{zu}$ verleihen. So wird es ihnen ermöglicht, ihre eigene epistemische Autorität zur Bewertung und Beurteilung der Serie zu stützen.

Die folgenden Fallbeispiele der der Praktik zugrunde liegenden Kollektion sind entlang dreier verschiedener Wissensquellen angeordnet. In Beispiel 17 wird Kontext- und Hintergrundwissen einbezogen, während in Beispiel 18 die $\mathrm{K}^{+}$Beteiligten Wissen aus einer anderen medialen Quelle einbeziehen, um die diskutierte Serie (medien)kritisch zu beurteilen. Anhand von Beispiel 19 (S. 143) wird schließlich veranschaulicht, wie das Expertenwissen abwesender Dritter in die Interaktion gebracht und zur Selbstpositionierung eingesetzt werden kann. Leitend für die Beschreibungen dieser Praktik ist die Frage: Wie positionieren sich $\mathrm{K}^{+}$-Beteiligte in Bezug auf die jeweilige serienexterne Wissensquelle und welche interaktiven Konsequenzen ergeben sich daraus?

\subsubsection{OUTLANDER - Bezug auf Kontextwissen}

(17) und dann (.) GOOgelt man so. 
$[\ldots]$

sind filme so die im MITelalter spielen oder sowas; sowas wie game of THRONES die haben (.) so $n$ mittelal, mit MITtelalterliche (.) äh:m;

(-) halt,

(.) mit MITtelalterlich dargestellt; =und das MAG ich;

DAV: ja w: wa äh was,

(.) WAS daran zum beispiel; weil GUCK mal ich,

(.) ich HASSe $[($ sowas; $)]$

ALI :

[oder Sowas?] wenn man, $(--)$ was ICH mag is, wenn man jetzt ne serie SCHAUT?

und sich danach noch geDANken macht und dann, (.) zum BEIspiel das is halt mittelmittelalterlich DARgestellt, =und dann (.) Googelt man so.

$(-)$ die namen von den HAUPTcharakteren oder die; zum beispiel hab ich OUTlander geguckt;

$=$ das is $n$

$=$ das is $n$.

(.) is ne SErie wo:,

((räuspert sich)) ne frau aus (--) dem KRIEGSzeit neunzehnhundert;

äh VIERzig?

da um den DREH, halt ähm: ;

(.) ähm: ;

in die vergangenheit fa äh REIST?

UNgewollt?

(.) und äh HALT;

(-) dann (.) zwEIhundert jahre VoRher dann;

LEBT bei.

(--) äh: :m;

(.) in SCHOTTland da in der nähe;

bei den CLANS und ähm, ENGland und äh: ;

$(-)$ bei bei England und SCHOTTland krieg so;

(.) da DAS noch mal so;

(--) MITbekommt;

(-) und dann,

(.) Googelt man das ganze und dann, sind halt da (.) hisTorische;

(--) ähm erEIGnisse;

die wirklich dann STATTgefunden haben;

=und das [ ( find ich so gut) 


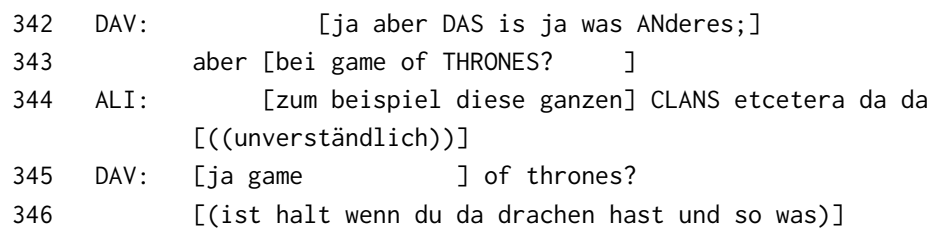

MNG13, 4:16-6:15

Nachdem Gisem, Igdir und Tufan erklärt haben, worum es in türkischen Serien geht, erläutert Ali zunächst seine Präferenz für historische Serien und solche, deren Settings im Mittelalter spielen (Z. 232-239). Es folgt eine an dieser Stelle ausgelassene Zwischensequenz zwischen ihm, David, Paul und Igdir, die vorrangig auf die evaluative Aushandlung von GAME OF THRONEs fokussiert ist (ab Z. 240, vgl. auch die Analysen in Abschnitt 9.1.1). Schließlich etabliert Ali die Serie OuTLANDER als Positionierungsobjekt, das er als Beispiel für seine Präferenz anführt. Er fasst den Plot der Serie zusammen und positioniert damit sich selbst als $K^{+}$und die anderen implizit als $K^{-}$in Bezug auf die Serie (Z. 307-344). Die Sequenz endet damit, dass David (erneut) GAME of THRONES als Positionierungsobjekt etabliert.

Ali rahmt seine Rekonstruktion mit einer positiven Bewertung, die sich auf tatsächliche historische Begebenheiten, die Serienhandlungen zugrunde liegen (Z. 306-314, 336-341), bezieht. Als externe Wissensquellen zieht er dafür internetbasierte Rechercheinstrumente (,googeln“, Z. 313, 337) heran. Er skizziert ein kurzes Szenario seines Rezeptionsverhaltens, wie er an seine Serienrezeption anschließend Hintergrundinformationen recherchiert (Z. 336-340). Seine Positionierung hebt er mit der generischen „man“- Formulierung (Z. 309-310) auf eine allgemeingültigere Ebene.

Auch seine Rekonstruktion ist genau auf diese Positionierung zugeschnitten: Anders als in anderen Rekonstruktionen, beispielsweise zu THE ORIGINALs in Beispiel 15, steht für ihn nicht die emotionale Erlebnisqualität im Vordergrund, sondern er organisiert seine Zusammenfassung um historische Daten, Ereignisse und Orte (Z. 319-320, 327, 330-333) herum. Die entsprechenden Turn-Konstruktionen weisen Reparaturen auf und sind gekennzeichnet von Alis Bemühen, Fakten zu nennen und mit seiner epistemischen Haltung Glaubwürdigkeit zu vermitteln. Zudem bewertet er nicht direkt die Serie positiv, sondern sein durch die Serie angeregtes Rezeptionsverhalten, also wenn man „sich danach noch geDANken macht“ (Z. 310) und dann erfolgreich historische Begebenheiten recherchieren konnte (Z. 340-341). Damit positioniert er sich selbst als jemand, dem Realitätstreue und potentielle Lerngelegenheiten besonders wichtig sind. Bei Betrachtung des interaktiven Geschehens fällt auf, dass niemand inhaltlich auf Alis Ausführungen eingeht. Er erhält weder Zustimmung noch Nachfragen oder Ergänzungen in Bezug auf 
die Serie. Stattdessen kontrastiert David wieder seine evaluative Haltung zu der ursprünglich von Ali als Favorit genannten Serie GAME OF THRONES (Z. 345-346), so dass die Sequenz danach zu diesem Positionierungsobjekt übergeht. Damit fechtet er weiterhin Alis Bewertung dieser Serie bzw. dieses Genres an.

Dass Ali Kontextwissen in dieser Sequenz in die Interaktion zur Positionierung einsetzt, spielt für den Fortgang der Gruppendiskussion eine wichtige Rolle. Seine mit dieser Praktik beanspruchte epistemische Autorität zur Beurteilung des ,Wahrheitsgehalts‘ und damit der Qualität einer Serie wird von David im weiteren Verlauf der Interaktion systematisch untergraben. Er positioniert Ali immer wieder als jemanden, der nicht stringent ist, da sich sein aus dieser Praktik abgeleitetes Bewertungskriterium für Serien - Realitätstreue (vgl. dazu auch Abschnitt 8.2.1) nicht auf GAME OF THRONES anwenden lässt (Z. 346) (vgl. auch Abschnitt 9.1.4).

\subsubsection{SCHWIEgERTOCHTER GESUCHT - Bezug auf mediales Wissen} (18) ich schreib darüber ja ne FACHarbeit,

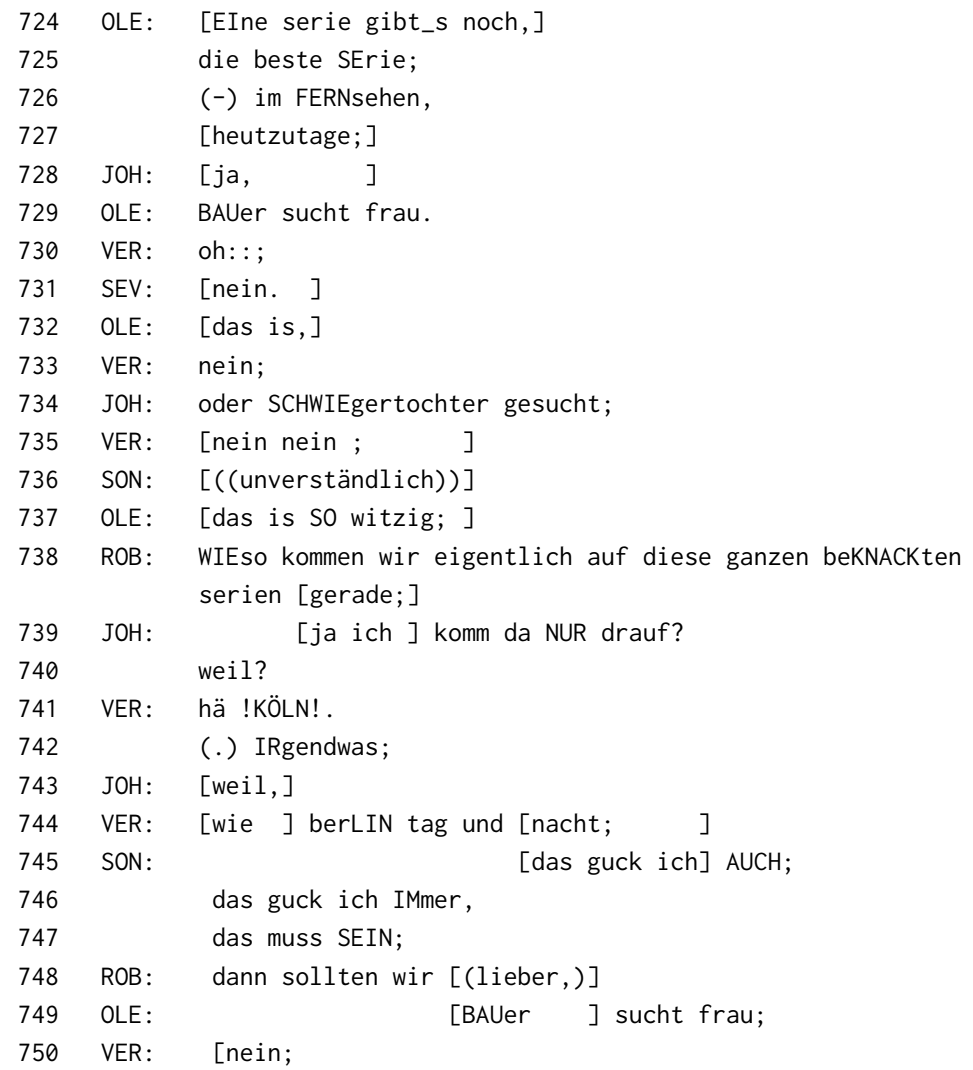


(.) nein;

OLE: [das ist natürlich die be!HIN!dertste] serie der welt; =ich hab die dann EInen abend mit TOM geguckt?

(-) wir haben uns so kaPUTT gelacht;

es war so WITzig;

$\mathrm{JOH}$ : aber es is so TRAUrig;

wie die leute die da MITmachen?

(.) bekommen keine $\mathrm{KOHle}$, werden über den TISCH gezogen bis zum geht nich mehr, und BLOß gestellt vor der [kamera; ]

OLE: $\quad[$ (wie bei)] jan BÖHmermann;

JOH: ja naTÜRlich;

OLE:

(.) aber wenn man da sich das [mal ANguckt?]

[war das die] mit der ver=

wo die ähm rtL für [das mit dem,]

$\mathrm{JOH}: \quad[$ (boah) ] ey.

OLE: da SCHWIEgersohn [da]

JOH: [ja] SCHWIEgers-

OLE: [TOCHter];

JOH: [ja ] schwiegerTOCHter gesucht;

=bei vera int VEEN,

die haben da $\mathrm{n}$ SCHWIEgersohn eingeschleust,

=alter?

(.) und der TYP der hat_n auftreten als wär der der größt, das größte OPfer, und der bekommt HUNdertfünfzig euro für dreißig DREHtage; $=<<$ hoch $>$ HUNdert [fünfzig; ]

OLE : [also ] wirklich,

die haben den DARgestellt die haben,

(.) ja ich mag ZÜge,

und dann [hat der immer so] äh SCHILDkröten gehabt,

JOH: [SCHILDkröten; ]

OLE: und DANN dann,

JOH: $\quad<<p>$ ja es is so geil; >

OLE: äh willst du auch mal ne ECHte schildkröte an (.) gucken und so;

$\mathrm{JOH}: \quad[(($ unverständlich $))]$

ROB: $\quad[(($ unverständlich $))]$

OLE: So und dann, dann kam rtl und hat im GANzen haus noch mehr äh; [PLÜSCH, ]

$\mathrm{JOH}: \quad[\mathrm{ja}:$; ]

OLE: SCHILDkröten verteilt,

$$
(-) \text { und [der HAT, ] }
$$

$\mathrm{JOH}: \quad[$ und so (PUZzle)]

OLE: der hat, 


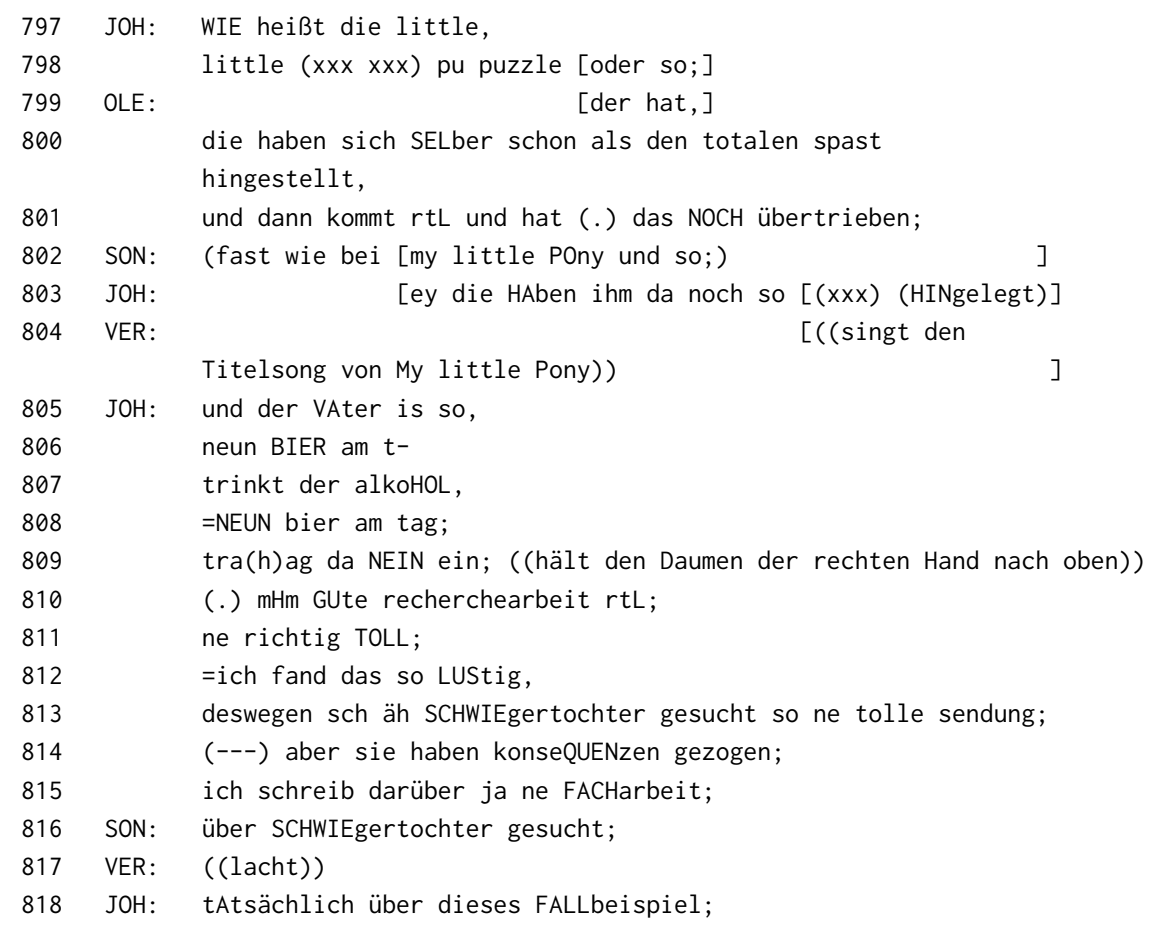

Johann und Ole positionieren sich in dieser Sequenz als $\mathrm{K}^{+}$in Bezug auf SCHWIEGERTOCHTER GESUCHT, wobei Johann die Serie in Anlehnung an BAUER SUCHT FRAU als Positionierungsobjekt (Z. 724-756) etabliert. Im weiteren Verlauf der Sequenz beansprucht Johann - und in etwas geringerem Maße auch Ole - die epistemische Autorität zur (kritischen) Bewertung der Serie (Z. 730-812). Dafür nimmt er Bezug auf die Sendung des Neo Magazin Royale vom 12. Mai 2016, in der Jan Böhmermann unter dem Hashtag \#verafake bekannt gab, einen Kandidaten in die Sendung eingeschleust zu haben. ${ }^{1}$ Johann beendet die Sequenz damit, dass er darauf hinweist, eine Facharbeit über diesen Vorfall zu schreiben (Z. 815-818). Die Gruppe thematisiert anschließend „Auto-Serien“ (vgl. Anhang IX: OST11, Z. 819).

Gemeinsam mit Ole bringt Johann das Wissen aus Böhmermanns Sendung sukzessive in die Interaktion. Sie markieren ihre Rekonstruktionen der Episode

1 Weiterführende Informationen dazu finden sich u.a. in folgendem Artikel der Süddeutschen Zeitung (Mai 2016): https://www.sueddeutsche.de/medien/verafake-bei-boehmermann-ende-dervorstellung-so-stellt-rtl-menschen-bloss-1.2993168 [zuletzt abgerufen: 06.04.2021]. 
und ihrer Produktionsbedingungen als Wissenskommunikation gegenüber den anderen Beteiligten (Z. 761-818). Darüber beanspruchen beide die epistemische Autorität zur Beurteilung von SCHWIEGERTOCHTER GESUCHT, indem sie bisweilen sehr detaillierte Wissenselemente, die ihnen aus Böhmermanns Sendung bekannt sind, teilen. Dazu zählen beispielsweise der kritikwürdige (Z. 757-760) und moralisch unverantwortliche (Z. 805-809) Umgang mit den Darsteller`innen (Z. 757-760), wörtliche Zitate aus den Dialogen der entsprechenden Sendung (Z. 785) sowie Inszenierungsverstärkungen des Produktionsteams, die nachträglich von Böhmermanns Team aufgedeckt wurden (Z. 739, 797, 800-801).

Darauf basierend nehmen Johann und Ole hinsichtlich ihrer Bewertung der Serie eine sehr komplexe Positionierung vor. So zeigt sich die Gruppe gegenseitig Wissen darüber an, dass sie diesen Serientyp eher populärkulturellen und gesellschaftlich weniger hoch angesehenen Serien ${ }^{2}$ zuordnen (Z. 730-755). Das lässt sich beispielsweise an Verenas wiederholten „nein“-Äußerungen $(\mathrm{Z} .733,735)$ oder Roberts Frage, wieso sie auf „diese ganzen beKNACKten serien“ kommen (Z. 738), erkennen. Dennoch signalisieren Johann und Ole vergnügliche Affinität dazu (Z.737, 755, 812). Mit der Praktik des Einbeziehens serienexterner Wissensquellen kritisieren sie allerdings zugleich klar die Produktionspraktiken des Fernsehsenders (Z. 810). Besonders deutlich wird das über die Ressource des Kontrastierens, die sich unter anderem in der evaluativen Paarsequenz zwischen Johann und Ole manifestiert: Ole bewertet die Serie BAUER SUCHT FRAU mit der abschließenden Formulierung „das war so WITzig“ (Z. 755), wobei seine vorherige Rahmung „das ist natürlich die be!HIN!dertste serie der welt; “ (Z. 752) auf das Vergnügen des (distanzierten) Rezipierens populärkultureller Serien verweist. Daran schließt Johann eine prosodisch und syntaktisch ähnlich gestaltete, aber semantisch kontrastive Bewertung „aber es is so TRAUrig;“ (Z. 756) an, die gleichermaßen den Auftakt zur oben beschriebenen Wissenskommunikation bildet.

Als zusätzliche Festigung seiner epistemischen Positionierung führt Johann zum Ende der Sequenz an, dass er sich mit den Hintergründen von ,\#verafake‘ in seiner Facharbeit auseinandersetzt. Er verleiht seinem epistemischen Status dadurch sowohl eine höhere Glaubwürdigkeit als auch eine stärkere Gewissheit. Diese Information wird als Doppeladressierung gerahmt. Einerseits legt die Verwendung von der Partikel ,ja“ (Z. 815) nahe, dass sein Facharbeitsthema den anderen Gruppenmitgliedern potentiell bekannt ist (siehe Abschnitt 4.3) und das Wissen an dieser Stelle für sie lediglich aktualisiert wird. Andererseits inszeniert er es - auch durch Blicke in die Kamera - gleichermaßen als relevante neue Information für die Forscherin.

2 Vgl. zum sog. Quality TV-Diskurs Abschnitt 2.1.3; auch Abschnitt 8.2.2. 
Damit beansprucht insbesondere Johann eine hohe epistemische Autorität zur (moralischen) Beurteilung der Serie. Zugleich vergemeinschaftet er sich aber auch mit anderen Gruppenmitgliedern über das gemeinsame Amüsieren darüber. Mit dieser doppelten Bewertung ,spielen“ Johann und Ole im Rahmen ihrer Positionierung, für die sie die Praktik des Einbeziehens serienexternen Wissens in Form von glaubwürdigem Meta-Wissen gekonnt einsetzen. Sie inszenieren sich als Rezipienten, die die Serie distanziert betrachten und dahinter liegende Strukturen kritisch durchschauen können, aber trotzdem Unterhaltungswert daraus ziehen (vgl. auch Ausführungen zu oppositionellen Lesarten in Abschnitt 2.1.3). Ähnlich wie in Beispiel 17 jedoch kommentieren die anderen Gruppenmitglieder den Verweis auf die externe Quelle kaum $(Z .788,802)$ und partizipieren somit insgesamt wenig an der Interaktion.

\subsubsection{THE WIRE - Bezug auf Expertenwissen}

(19) dann kann es sein dass es SCHWErer ist dass man sich da rEInfindet;

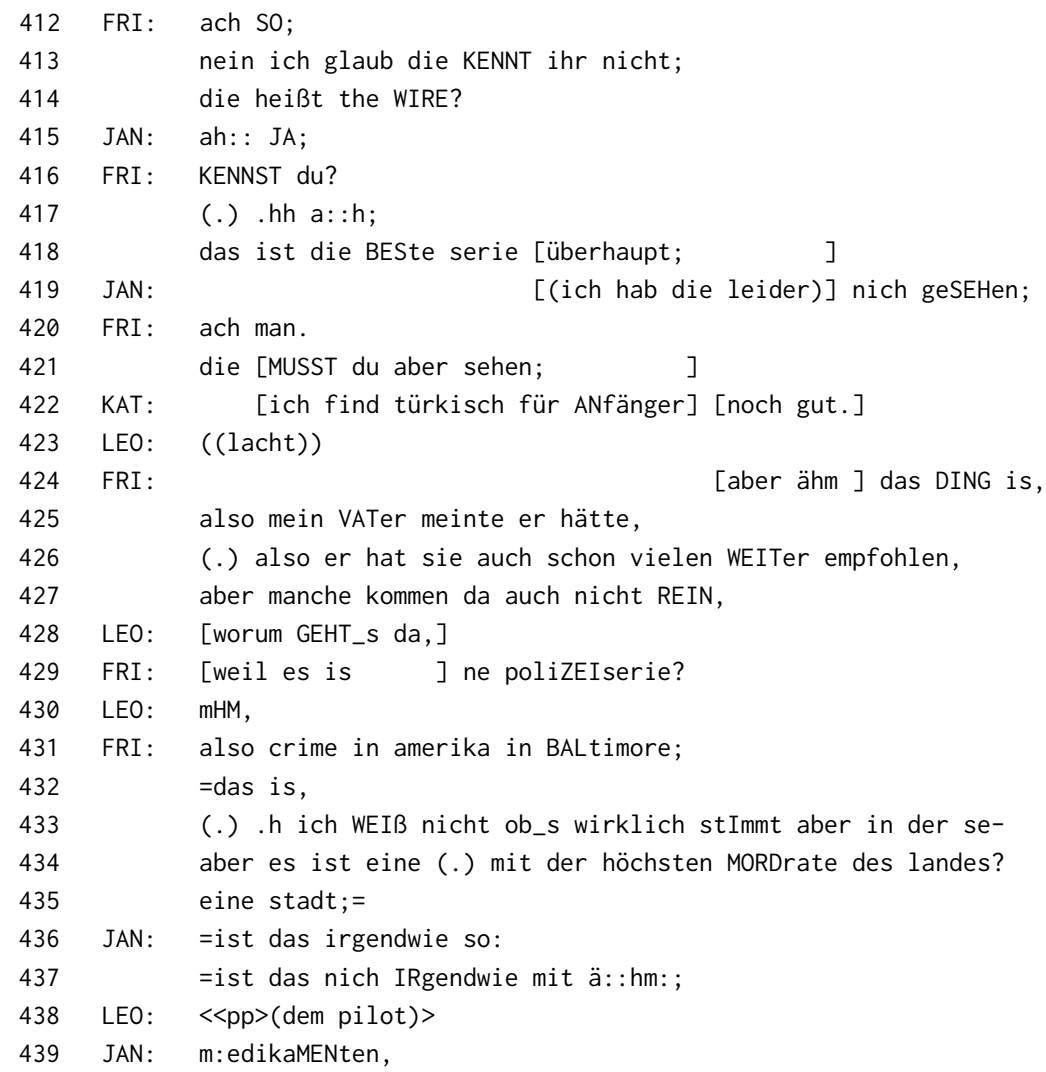


=also es sind irgendwie DROgen oder sowas?

$(--)$ nich,

FRI : ((lacht)) [also] ja AUCH.

JAN: $\quad[$ nee; $]$

FRI: [aber das ist jetzt nicht der !HAUPT!bestandteil] der serie;

JAN: [ah pf: (-) ich DACHte.

FRI: es geht EINfach,

(.) um das leben verschiedener charaktere in dieser STADT, =also zum einen die polizisten, dann eben die JuNkies und die:;

(.) DROgendealer?

JAN: hm_hm;

FRI: $\quad u:$ nd es IST eben;

(.) WIRKLich sehr realitätsnah. und das macht die serie so GUT?

(.) aber DAdurch wenn man eben, (-) nicht so sehr da DRIN ist;

also jetzt wie wenn man zum beispiel mein VAter kennt sich halt mit polizeiarbeit gut aus weil er polizIst ist,

JAN: hm_hm.

FRI: aber wenn man das jetzt (.) NICHT kann, dann kann es sein dass es SCHWErer ist dass man sich da rEInfindet;

weil man eben nicht MERKT wie;

(--) realiTÄTstreu die serie eben ist; und das (.) ist eigentlich DAS was sie gUt macht.

JAN: ist ist das so ne case of the WEEK (.) folge, =also se serie also wo: in jeder folge sozusagen ein neuer FALL drankommt?

FRI: naJA also es, (.) ähm nee EIgentliches geht immer um die (.) großen DROgenbosse; $(-)$ die_s (.) $[<<p>$ in der (stadt so GIBT. $>)]$

JAN: [oke aber es ] ist jetzt nicht so wie MONK oder so dass da jed, in [jeder folge] so_n-

FRI : [nein. ]

JAN: oke nee;

FRI: es baut alles auf einander AUF [(immer;)]

JAN: [gut; ]

KAT: aber es gibt so ne richtig gute serie ich glaub die heißt sarah lund, 
Friederike wurde zuvor aufgefordert, ihre Lieblingsserie zu nennen. Sie beginnt die Etablierung des Positionierungsobjekts THE WIRE mit der abgeschwächten Zuschreibung eines niedrigen epistemischen Status an die anderen Teilnehmenden (,nein ich glaub die kennt ihr nicht“, Z. 413), der dann kurz verhandelt wird (Z. 415421), und markiert damit ihren eigenen $\mathrm{K}^{+}$-Status. Nach Leons Aufforderung zur Rekonstruktion (Z. 428) fasst Friederike die Handlung der Serie zusammen (Z. 429453) und bewertet sie mit Verweis auf den Beruf ihres Vaters positiv (Z. 454-463). Anschließend folgt ausgelöst durch Jans Nachfrage eine Sequenzerweiterung, in der Friederike weitere Wissenselemente mit der Gruppe teilt (Z. 464-475), und Katharina beendet die Sequenz, indem sie mit SARAH Lund ein neues Positionierungsobjekt etabliert (Z. 476).

Friederike beurteilt die Serie mittels der extreme case-Formulierung „BESte serie überhaupt“ (Z. 418) als stark positiv. Als Begründung führt sie an, dass die Serie sehr „realitätsnah“ (Z. 453-454) bzw. „realitätstreu“ (Z. 462-463) bezüglich der Darstellung der Lebensverhältnisse verschiedener Figuren (Z. 446-450) sei. Ihre epistemische Autorität zur Beurteilung und adäquaten Einschätzung dieses Bewertungskriteriums speist sich dabei insbesondere aus dem Beruf ihres Vaters als Polizist, den sie als Experten für ihre epistemische Positionierung hinzuzieht. Den Grundstein dafür legt sie schon zu Beginn der Sequenz, als sie die manchmal nicht von Erfolg gekrönte Empfehlung der Serie durch ihren Vater mit der Kategorisierung der Serie als „poliZEIserie“ (Z. 429) begründet. Erst später stellt sie den Zusammenhang zu seinem Beruf explizit her (Z. 475).

Sie stellt die Tatsache, dass sie Zugang zu dieser Wissensquelle hat, einerseits als individuelle Besonderheit - als Distinktionsmittel -, andererseits auch als generell notwendig zur (verstehenden) Rezeption und einer darauf beruhenden adäquaten Einschätzung der Serie heraus. Das wird daran deutlich, dass sie mehrfach Kontrastieren als Ressource zur Positionierung einsetzt, indem sie anderen Rezipient*innen, denen das berufsbedingte Expertenwissen ihres Vaters fehlt und damit implizit auch den anwesenden Gruppenmitgliedern -, eine potentiell abweichende Bewertung zuschreibt (Z. 425-427, 455-463). Sie selbst positioniert sich also in Kongruenz mit der Bewertung ihres Vaters in Bezug auf die Serie. Durch den Verweis auf sein Wissen und seine Urteilskraft verleiht sie so ihrer eigenen positiven Bewertungsbegründung qua externer Autorität Glaubwürdigkeit (als "good authority“ nach Pomerantz 1984; vgl. Abschnitt 4.3).

Auch in diesem Beispiel gehen die übrigen Teilnehmenden nicht explizit auf die hinzugezogene Wissensquelle ein und es bleibt bei Minimalratifizierungen (Z. 451, 458) als Reaktion auf Friederikes Positionierung. Anders als in den Beispielen 17 und 18 jedoch stellt Jan mehrere Informationsfragen (Z. 464-465, 470-471), die - nachdem Friederike ihn zunächst ebenfalls als $K^{+}$behandelt hatte (Z. 416- 
421) - seinen eigenen $\mathrm{K}^{-}$-Status etwas hochstufen, indem er Kenntnis einzelner Wissenselemente signalisiert (vgl. dazu auch Abschnitt 7.2.1).

\subsubsection{Zusammenfassung: Die Praktik Einbeziehen serienexterner Wissensquellen}

Die Positionierungspraktik des Einbeziehens serienexterner Wissensquellen wird in Abbildung 7.2 schematisch visualisiert (siehe zur Erläuterung Abschnitt 6.4) und lässt sich folgendermaßen beschreiben:

- Strukturstelle(n): Ein oder mehrere Gruppenmitglieder (A) positionieren sich als diejenigen mit der höchsten epistemischen Autorität (eA) in Bezug auf die Bewertung der Serie. Sie schließen eine über mehrere Turns hinweg gestaltete Begründung ihrer Bewertung an.

- Ausgestaltung: Die $\mathrm{K}^{+}$-Sprechenden ziehen als Begründung für die Bewertung mittels Rekonstruieren, Kategorisieren und Kontrastieren eine externe Wissensquelle hinzu und positionieren sich selbst in Relation zu dieser als glaubwürdig behandelten Quelle. Es finden kaum interaktive Aushandlungen oder ein Aufgreifen solcher Wissensquellen seitens der $\mathrm{K}^{-}$-Beteiligten (B) statt. Sie werden nach ihrer Einführung in die Interaktion weitgehend ,hingenommen'.

- Interaktive Funktion(en): Die Quellen dienen dazu, die eigene epistemische Autorität zur Bewertung als glaubwürdig zu markieren und damit zu unterstützen. So werden sie von den Teilnehmenden einerseits als ,Lieferanten' für Kriterien zur Bewertung behandelt. Andererseits stellen sie zugleich Wissenselemente zur Verfügung, die es den Beteiligten erlauben, Serien entlang dieser Kriterien zu bewerten. Die Vergemeinschaftung über geteilte Bewertungen und der Abbau epistemischer Asymmetrie gegenüber $\mathrm{K}^{-}$-Beteiligten können auch Funktionen dieser Praktik sein (vgl. v.a. Beispiel 19), spielen aber tendenziell eher eine untergeordnete Rolle.

Das Hinzuziehen serienexterner Wissensquellen stellt eine Praktik dar, deren zentraler Aspekt in doppelter Hinsicht die Glaubwürdigkeit ist. Nicht nur die Positionierenden können über eine Glaubwürdigkeit transportierende epistemische Haltung ihren Anspruch auf Expertise - im Sinne eines höheren Wissens und einer durch externe Autorität gestützten Bewertung - geltend machen, sondern auch an die Serie selbst wird dieses Kriterium angelegt. Für die Positionierung von Ali (Beispiel 17) und Friederike (Beispiel 19) ist der Realitätsbezug die Begründung für ihre positive Bewertung. Bei beiden Serien handelt es sich um fiktionale Serien, so dass die in Beispiel 18 thematisierte Serie SCHWIEGERTOCHTER GESUCHT als Doku-Soap einen Sonderstatus einnimmt. Selbstverständlich enthält auch diese 
Serie fiktionale Anteile, sie vermittelt jedoch durch die Art und Weise ihrer narrativen Gestaltung sowohl Anschein als auch Anspruch der Realitätstreue. Insgesamt scheint sich diese Praktik seltener bei der Thematisierung von Serien mit fantastischen Elementen zu finden; GAME of THRonEs stellt eine Ausnahme dar (vgl. Abschnitt 9.1.4). Indem Johann und Ole diesen Anspruch dekonstruieren, messen sie also die Serie ebenfalls an dem Kriterium der Glaubwürdigkeit (vgl. dazu auch Abschnitt 8.2.1). Deutlich wird an dieser Praktik, dass die Rezipientinnen nicht nur Unterhaltung, sondern auch Wissenszuwachs und (kritische) Einordnung der Serien relevant setzen.

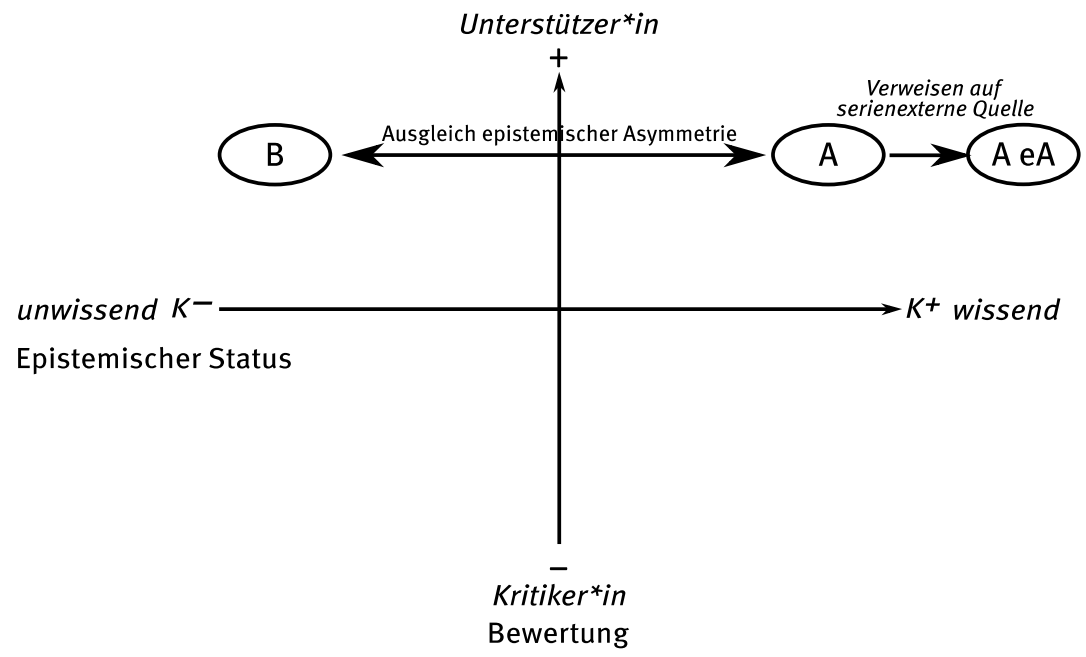

Abb. 7.2: Schematische Darstellung der Praktik Einbeziehen serienexternen Wissens

\subsubsection{Absprechen von fremder epistemischer Autorität zur Bewertung bei K-}

In den Daten zeigt sich, dass abweichende Bewertungen nicht notwendigerweise dispräferiert sind, sondern für das Ziel der Distinktion funktionalisiert werden können. Zwar sind die Erstbewertungen häufig bestätigungseinladend (vgl. auch die tabellarische Übersicht nach Raymond und Heritage 2006 in Abschnitt 4.3), allerdings finden die Teilnehmenden bisweilen auch Vergnügen im Dissens (vgl. Abschnitt 7.3.3). Wenn sich im Laufe der Sequenz jedoch herausstellt, dass die abweichende Zweitbewertung auf (zu) wenig Wissen basiert und eine Person somit unrechtmäßig Expertise beansprucht, greifen $K^{+}$-Beteiligte auf die Praktik 
des Absprechens der fremden epistemischen Autorität zur Bewertung zurück. Sie stellen also die Positionierung einer anderen Person in Frage (,challenging“ einer Position nach Bücker 2013, vgl. Merkmal (3) in Abschnitt 3.3).

Die folgenden Beispiele sind entlang dreier verschiedener, jeweils negativer Bewertungsausrichtungen angeordnet. Anhand von Beispiel 20 wird veranschaulicht, wie die epistemische Autorität nach einer subjektiv formulierten Bewertung, die keine Allgemeingültigkeit beansprucht, abgesprochen wird. Beispiel 21 basiert auf einer moralischen Bewertung, während die Bewertung in Beispiel 22 (S. 154) auf stereotypischen Vorstellungen und pauschalen Urteilen basiert. Leitend für die Beschreibungen dieser Praktik ist die Frage: Auf welcher Grundlage bewerten $\mathrm{K}^{-}$-Beteiligte eine Serie negativ, so dass die $\mathrm{K}^{+}$-Beteiligten ihnen die epistemische Autorität zur Beurteilung absprechen, und welche interaktiven Konsequenzen ergeben sich daraus?

\subsubsection{MORD MIT AUSSICHT - Zuschreiben von $\boldsymbol{K}^{-}$nach subjektiver Bewertung (20) du musst $n$ bisschen MEHR sehen;}

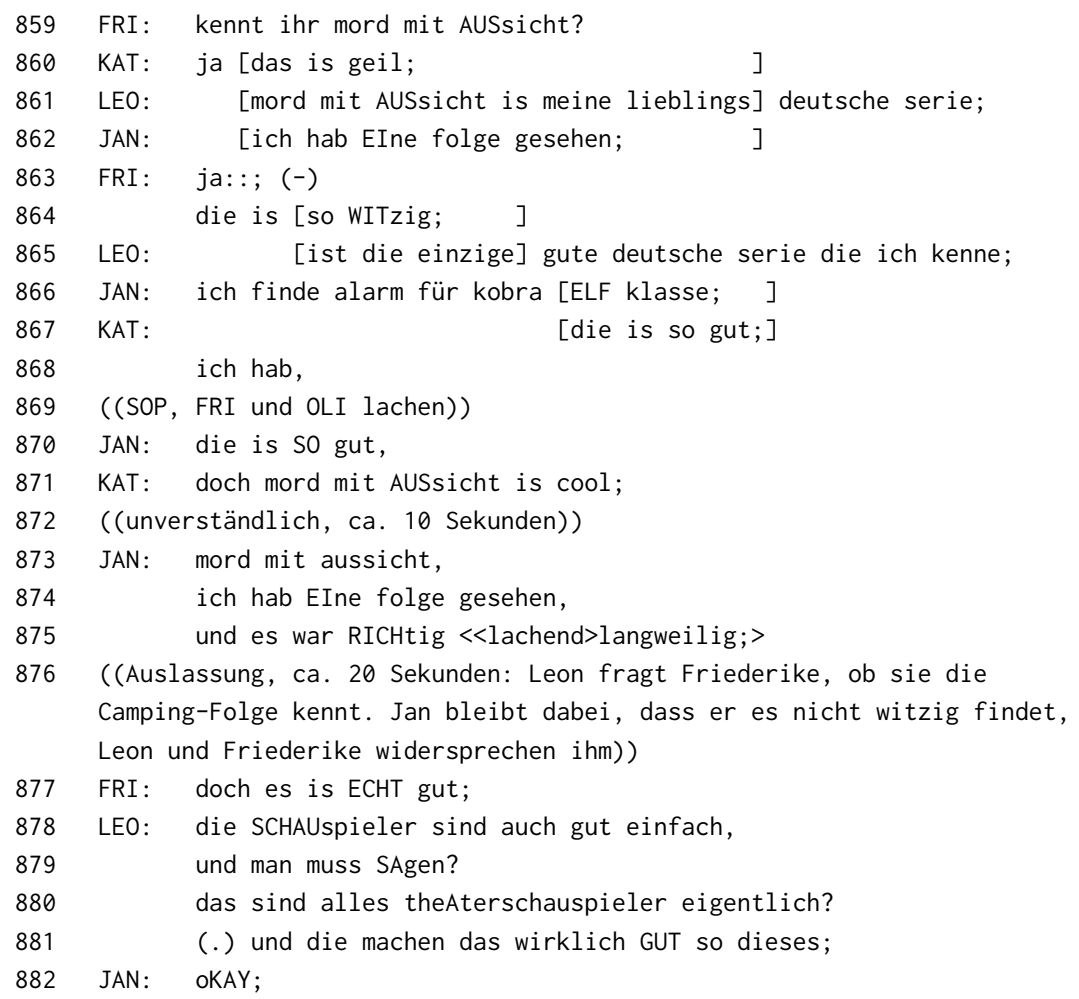




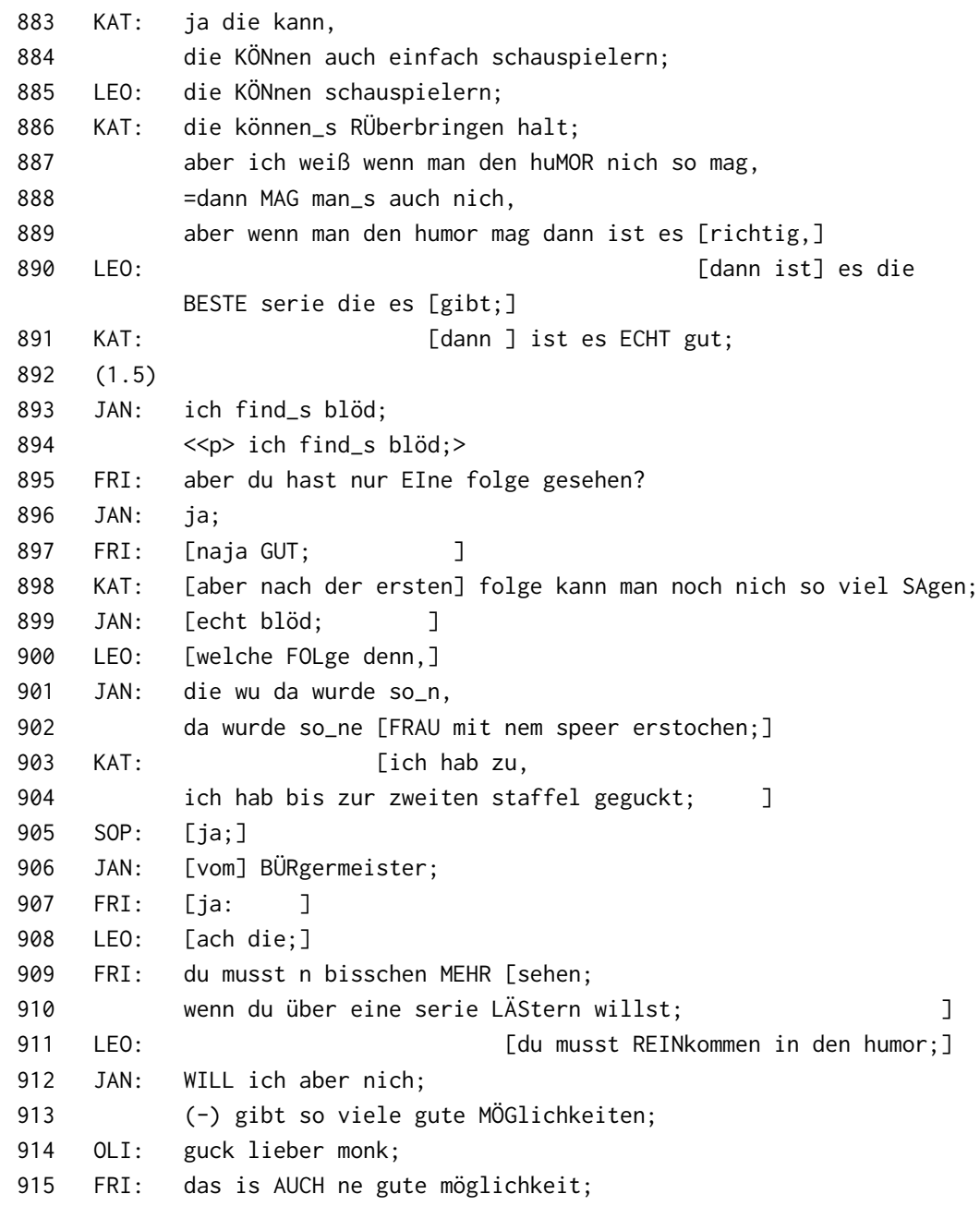

Friederike, Katharina und Leon stimmen darin überein, dass die Serie MORD MIT Aussicht die beste (deutsche) Serie (Z. 859-871) sei, wohingegen Jan die Serie mehrfach negativ bewertet (Z. 873-875, 893-984). Nachdem sich Leon und Friederike wechselseitig an deren $\mathrm{K}^{+}$-Status orientieren und sich der geteilten Kenntnis einer Episode vergewissern (Z. 876) , führen die Unterstützer^innen an Jan adressierte Begründungen für die Qualität der Serie an (Z. 878-892). Als Jan jedoch bei seinem negativen Urteil (Z. 893-894) bleibt, schließt sich eine Aushandlungsse- 
quenz an, in der die Teilnehmerinnen ihm zu verstehen geben, mit diesem Wissen keine fundierte Bewertung vornehmen zu können (Z. 895-915).

Jans Bewertung basiert weder auf einer besonders negativen Attribuierung (wie „scheiße“ o. Ä., vgl. Beispiel 21) noch rahmt er sein Urteil als objektiv bzw. allgemeingültig (vgl. Beispiel 22). Er formuliert stattdessen seine Bewertung als subjektive Meinung, die auf der Rezeption einer Episode basiert (Z. 874-875, 893), womit er sich zugleich als eher $K^{-}$positioniert. Die Lachpartikeln (Z. 875) schwächen die - offenbar hier durchaus als dispräferiert markierte - widersprechende Zweitbewertung ab. Seine epistemische Positionierung wird zunächst nicht thematisiert, stattdessen argumentieren Katharina und Leon für die Qualität der Serie, indem sie gemeinsam als zusätzliches Wissenselement die Theatererfahrung der Schauspieler*innen und daraus resultierend deren performative Kompetenz positiv betonen (Z. 878-886). Jans „oKAY; “ (Z. 882) deutet jedoch an, dass er nicht überzeugt wurde. Katharina kommt ihm mit der schlichtenden Äußerung entgegen, dass eine Präferenz der Serie offenbar auch mit einem spezifischen Humorverständnis einherginge (Z. 887-891), was Leon bestätigt (Z. 890), Jan jedoch nicht kommentiert.

Als Jan daraufhin bei seiner negativen Bewertung bleibt (Z. 983-984), greift Friederike schließlich seine eingangs getätigte epistemische Positionierung in Form einer bestätigungseinladenden Frage wieder auf (Z. 895), die er auch ratifiziert (Z. 896). Erst daraufhin sprechen ihm Katharina und Friederike gemeinsam die epistemische Autorität zur Bewertung bei niedrigem epistemischen Status mittels folgender Formulierung ab - wenn auch in relativ abgeschwächter und damit face-wahrender Form: „aber nach der ersten folge kann man noch nich so viel SAgen;“ (Z. 898). Parallel dazu bekräftigt Jan jedoch seine negative Bewertung und Katharinas Äußerung wird interaktiv zunächst nicht weiter relevant. Es schließt sich eine Zwischensequenz an, in der Jan auf Leons Frage hin den Inhalt der von ihm rezipierten Episode zusammenfasst (Z. 900-908). Es ließe sich vermuten, dass Leon damit eine Erklärung für Jans Urteil sucht, allerdings erfolgt im Anschluss keine Verständigung über die Bewertung dieser Episode.

Friederike verschiebt nun die potentiell face-bedrohende Situation (vgl. Beispiele 21 und 22) auf eine humorvolle Ebene: „du musst $n$ bisschen MEHR sehen; wenn du über eine serie LÄStern willst“ (Z. 909-910). Damit adressiert sie ihn direkt im Unterschied zu Katharina, die ihn zuvor mit ihrer generischen „man“Formulierung (Z. 898) eher implizit angesprochen hat. Jan reagiert jedoch nicht auf Friederikes scherzhaft verpackten Vorwurf, während er Leons Ergänzung „du musst REINkommen in den humor; “ (Z. 911) ohne Hedging ablehnt (Z. 912).

Trotz diverser potentiell heikler Momente, in denen Jans epistemische Autorität zur Beurteilung der Serie nach Rezipieren von nur einer Folge in Frage gestellt wird, gelingt es der Gruppe, kooperativ und weitgehend face-schonend durch 
die Sequenz zu manövrieren. Die $K^{+}$-Teilnehmenden zeigen ihm interaktiv Möglichkeiten auf, seine Bewertung abzuschwächen - Zugeben eines differierenden Humorgeschmacks (Z. 887-889) oder Eingrenzen seiner negativen Bewertung auf die erste Episode mit Offenheit für eine potentielle Qualitätssteigerung, sobald er mehr gesehen hat (Z. 895-911) -, die er jedoch nicht wahrnimmt. Mit Olivers Vorschlag, „lieber monk“ zu schauen (Z. 914), wird die Sequenz schließlich unproblematisch und bestätigend beendet (Z. 915). Eine mögliche Erklärung dafür wäre einerseits Jans durchgängige Rahmung der Bewertung als aus seiner subjektiven Position heraus gesprochen, die keine universelle Gültigkeit beansprucht. Darin unterscheidet sich dieser Fall von denen anderer in der Kollektion. Eine andere Erklärung wäre die generelle kooperative und höfliche Atmosphäre, die diese Gruppe fast durchgängig kreiert. Für die Ausgestaltung der Praktik des Absprechens epistemischer Autorität im Rahmen der hier vorliegenden Daten erscheint dieser Aspekt sicherlich nicht unwichtig.

\subsubsection{GAME OF ThRONES - Zuschreiben von $\boldsymbol{K}^{-}$nach moralischer Bewertung (21) siehst du dann WEISST du_s nich;}

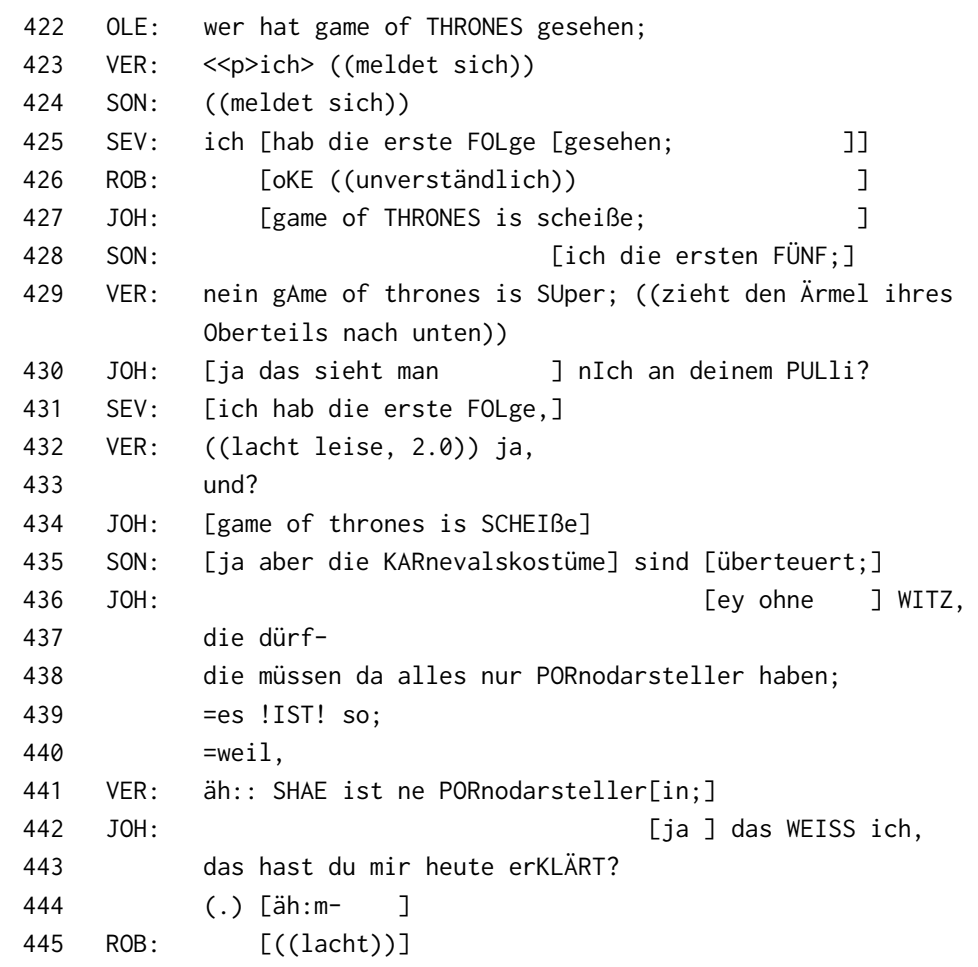




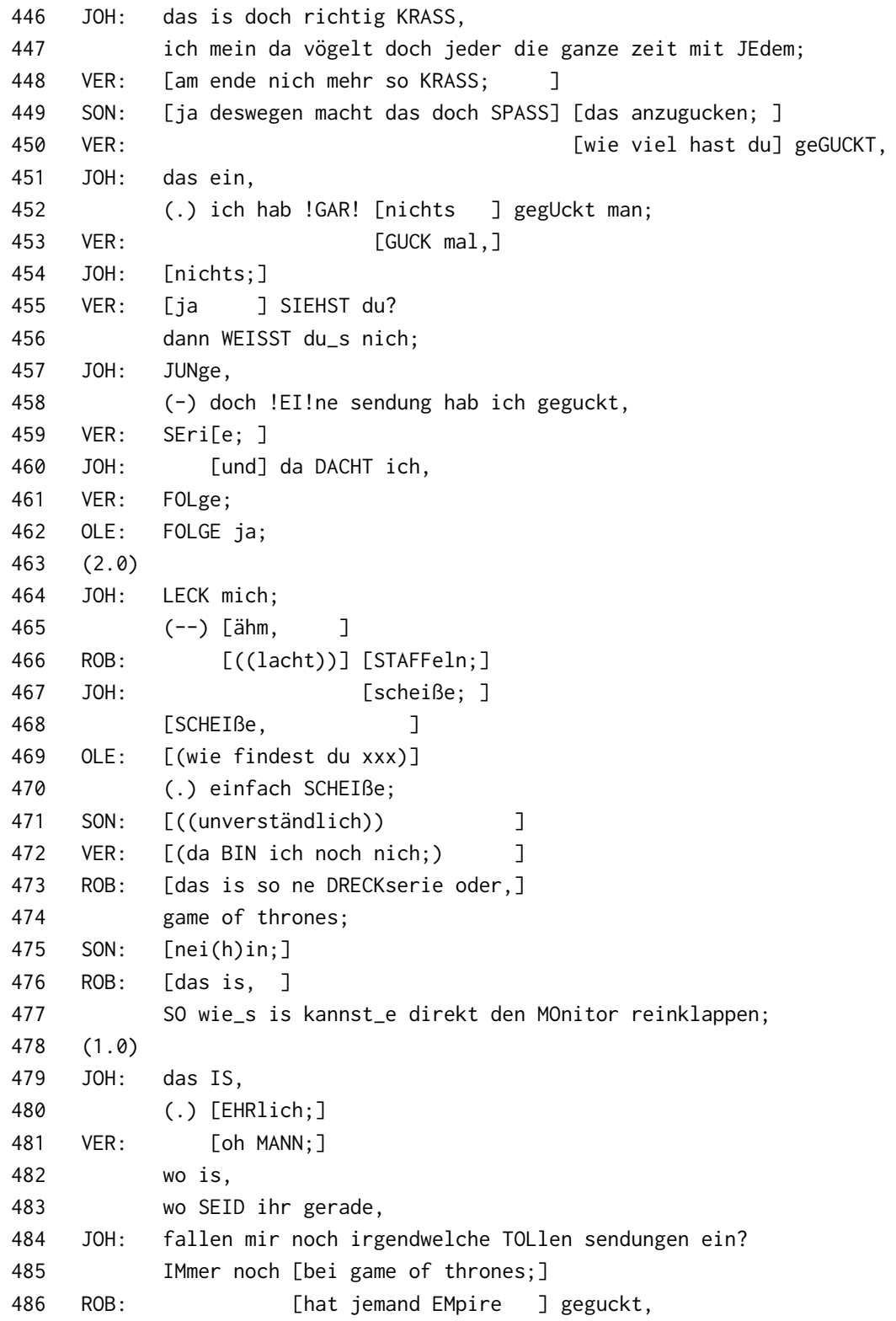

In dieser Sequenz wird zunächst die Frage geklärt, wer die Serie GAME OF THRONES kennt und sie in welcher Art und Weise bewertet (Z. 422-434). Johann gibt als 
Ursache für seine negative Bewertung die häufige Thematisierung und Darstellung von Sex in der Serie an, was daraufhin zum Gegenstand interaktiver Aushandlung wird (Z. 435-449). Als erkennbar wird, dass er keine Episode der Serie gesehen hat (Z. 450-454), spricht ihm Verena die epistemische Autorität zur Beurteilung ab (Z. 453, 455-456). Es folgt eine Korrektursequenz hinsichtlich des Begriffs zur Bezeichnung einer Episode (Z. 455-466, vgl. dazu auch Abschnitt 8.1.1). Während Johann, Sonja und Robert die Serie weiterhin negativ bewerten, diskutieren Ole und Verena die Serie inhaltlich (Z. 467-481). Die Sequenz wird mit der Suche nach einem neuen Positionierungsobjekt (EMPIRE) abgeschlossen (Z. 482-486).

Als Ole den epistemischen Status der anderen bezüglich der Serie GAME of Thrones erfragt (Z. 422), melden sich Verena und Sonja bzw. verweisen leise auf sich selbst (Z. 423). Johann stuft die Serie sehr negativ ein (Z. 427), woraufhin Verena Blickkontakt zu ihm aufnimmt und seine Äußerung mit einer explizit positiven Bewertung (Z. 429) kontrastiert. Zugleich zieht sie den Ärmel ihres Oberteils nach unten (Z. 329). Diese Handlung nutzt Johann dazu, das Kleidungsstück als Ressource in die Interaktion einzubeziehen, indem er ihre Bewertung wiederum unter Bezugnahme auf ihren Pullover ironisch kommentiert (Z. 430). Bei dem Pullover handelt es sich um Game-of-Thrones-Merchandise, so dass die Aussage unter Einbezug dieser Information verständlich wird: Verena wird positioniert (und positioniert sich selbst) als Fan der Serie, wodurch ihre positive Einschätzung der Serie ,selbstverständlich“ erscheint.

Gegen diese Positionierung argumentiert Johann im Laufe der Sequenz mehrfach, indem er seine negative Bewertung damit begründet, dass alle Schauspieler`innen in der Serie „PORnodarsteller“ seien (Z. 438). Dieser Aussage verleiht er mit einer sehr starken Betonung eine hohe epistemische Gewissheit (Z. 439). Seine ansetzende Begründung (Z. 440) unterbricht Verena. Sie ignoriert seine negative Bewertung und stimmt ihm stattdessen inhaltlich insofern zu, als sie einwirft, Shae sei eine Pornodarstellerin (Z. 441). ${ }^{3}$ Den Johann damit implizit zugeschriebenen $\mathrm{K}^{-}$-Status weist er mit Referenz auf ein gemeinsames Gespräch zurück (Z. 442-444). Insgesamt kommuniziert er über seine evaluative Haltung explizit, dass er über die epistemische Autorität zur (moralischen) Beurteilung der Berufe der Schauspieler`innen (vgl. dazu auch die Analyse in Abschnitt 9.1.1) bzw. der augenscheinlichen Fokussierung auf Sex in der Serie verfügt (Z. 446-447). Verena und Sonja ratifizieren seine Behauptung und widersprechen ihm nicht. Sie gehen interaktiv jedoch unterschiedlich damit um: Während Verena sie als für aktuelle Folgen nicht mehr zutreffend erklärt (Z. 448), deutet Sonja den von

3 Shae ist der Name einer Figur in Game of Thrones, die von Sibel Kekilli gespielt wird. Siehe auch Abschnitt 4.2 zur Bedeutung des Einbezugs von Hintergrundwissen für die Analyse. 
Johann angeführten negativen Bewertungsgrund positiv um (Z. 449) (vgl. dazu auch die weiterführenden Analysen in Abschnitt 9.1.4).

Schließlich stellt Verena in zwei Schritten seine epistemische Autorität zur Bewertung der Serie in Frage. Zunächst fragt sie ihn, wie viel der Serie er gesehen habe (Z. 450). Als deutlich wird, dass er keine Episode kennt (Z. 452, 454), spricht sie ihm die Berechtigung zur Bewertung mit folgender Formulierung ab: „ja SIEHST du? dann WEISST du_s nich;“ (Z. 455-456). Johann relativiert als Reaktion auf Verenas Aussage seinen epistemischen Status dahingehend, dass er „eine Sendung“ (Z. 458) gesehen habe, seine Bewertung verändert er jedoch nicht (Z. 467, 468), auch nicht in den nachfolgenden Sequenzen, in denen es um GAME of Thrones geht. Seine Antwort auf Verenas Reparatur des Begriffs fällt dagegen mit der Äußerung „LECK mich“ (Z. 464) wenig face-schonend - wenn auch scherzhaft gerahmt und damit abgeschwächt - aus.

Analog zu Beispiel 20 versuchen die anderen Gruppenmitglieder zunächst noch, Johanns negative Bewertungen zu relativieren oder umzudeuten sowie ihm ,Brücken' zu einer Abschwächung seiner Bewertung zu bauen - solange, bis offenbar ein bestimmtes Maß erreicht ist. Dann weisen sie sein Urteil zurück und stellen seinen epistemischen Status in Frage. Johanns Bewertung speist sich hier aus einer moralischen Positionierung heraus. Er macht sich über Sex als scheinbar einziges Thema und Handlungselement der Serie lustig und empört sich - teils scherzhaft gerahmt - darüber. Er stuft seine Bewertung abschließend nicht zurück, sondern modifiziert lediglich seine epistemische Haltung. Damit entspricht seine Art und Weise der Positionierung auch der, die er in anderen Sequenzen vornimmt. Hat er einmal eine Serie (extrem) bewertet, weicht er von dieser Bewertung nicht mehr ab (vgl. u.a. Beispiel 18).

\subsubsection{BoLlYwOOD - Zuschreiben von $K^{-}$nach pauschalisierender Bewertung (22) GUCKT ihr kein bollywood;}

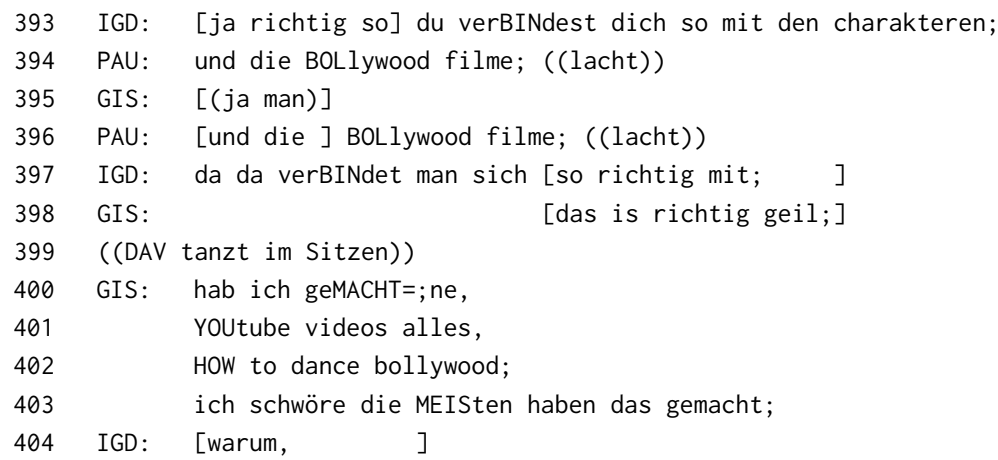




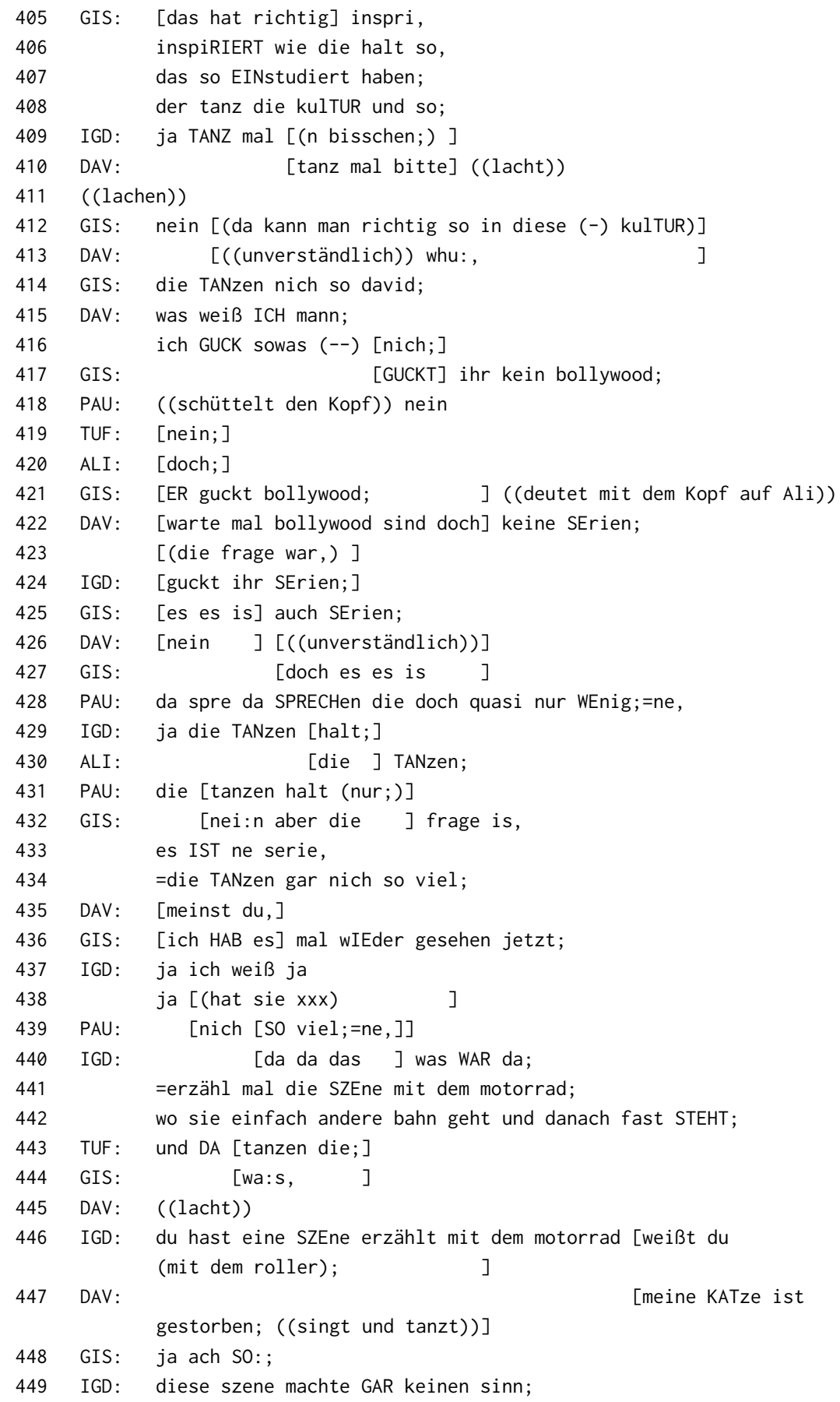


450

451

452

453

454

455

456

457

458

459

460

461

462

463

464

465

466

467

468

469

470

471

472

473

474

475

476

477

478

479

480

481

482

483

484

485

486

487

488

489

490

491

492

493

494

495

guck mal erZ̈̈HL die;

GIS: guck mal,

ja war richtig beHINdert;

=du hast so ne serie?

(.) ein MANN und eine frau so;=ne, das is so HASSliebe bei denen;

also die HASsen sich so gegenseitig und dann is es die große LIEbe und so;

die gehen so (in die stadt dann regelmäßig,)

aber es is halt INdien;

n DORF ;

ist was ANderes

DAV: [so wie in der türKEI;]

GIS: [und dann,

und äh das mädchen fährt einfach so;

(.) entGEgegen,

entgegengesetzt der verkehr,

des verKEHRS?

IGD: GEISterfahrer ist sie;

GIS: also sie fährt einfach So,

und eigentliche fahrt [wäre dann eben, ]

TUF : $\quad$ [ja ((unverständlich))]

PAU: [ja ja ]

DAV: [ja klar ]

ALI: $\quad$ [ja ja GEISterfahrer; ]

GIS: $\quad$ und der REIche mann?

der reiche mann ist dann im AUto,

=hinter dem AUto und sie: ;

(-) fährt ganz langsam so AN?

und das hat so $n$ KRATzer und so;

und DAS is meint er;

IGD: das das das hat [GAR keinen kratzer; ]

TUF: [(und dann tanzen die;]

IGD: [warum warum das macht gar keinen sinn]

GIS: [die TANzen nicht mann;

die tanzen in dieser serie nur VIER [fünf mal; ]

PAU: [(und wie viele] folgen,)

GIS: das sind nur zwei FOLgen;

IGD: in zwei folgen tanzen die vier fünf mal,

(3.0)

GIS: das is eigentlich [((unverständlich))]

so die tanzen so die tanzen WEnig;

IGD: $\quad[(($ unverständlich $))]$

GIS: nur auf HOCHzeiten tanzen die,

auf FEStivals,

und mehr nich;

IGD: ach so oke; 


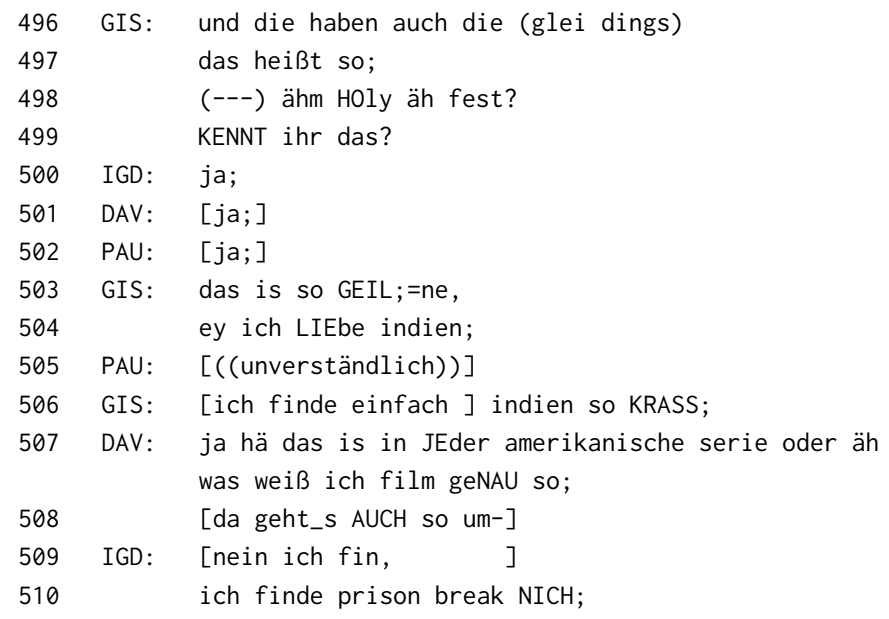

Diese Sequenz schließt an eine Diskussion um das emotionale Einfühlen in fiktive Figuren (vgl. Abschnitt 8.1.4) an (Z. 393-397). Zuvor hatte Gisem mehrfach erfolglos versucht, das Thema BoLLywood als Positionierungsobjekt ${ }^{4} \mathrm{zu}$ etablieren. Dieses Thema greifen Paul und Igdir nun scherzhaft gerahmt auf im Hinblick auf die Identifikationsmöglichkeit mit Figuren in Bollywood-Filmen (Z. 394-397). Gisem nutzt die Gelegenheit für eine erneute positive evaluative Positionierung (Z. 398), woraufhin sich zunächst eine Aushandlungssequenz zu Tanzpraktiken in Bollywood-Produktionen (Z. 399-416), dann zu der Frage, ob Bollywood als relevant für die zu bearbeitende Gruppenaufgabe gilt (Z. 417-439), entfaltet. Igdir fordert Gisem auf, eine Szene aus einer Episode zu rekonstruieren, an der er sich partiell beteiligt (Z. 440-482). Schließlich erläutert Gisem, zu welchen Gelegenheiten die Figuren in Bollywood-Produktionen tanzen (Z. 483-506). Mit der Initiierung eines Vergleichs zu amerikanischen Serien beendet David die Sequenz (Z. 507-508).

Gisem muss als wissende Unterstützerin ihre $\mathrm{K}^{+}$-Position und positive Bewertung durchgängig gegen stereotypische Vorstellungen (,,in Bollywood-Filmen wird die ganze Zeit nur getanzt“; vgl. Z. 399, 428-429, 443, 447, 481) und damit implizit negative und amüsierende Bewertungen der anderen Teilnehmer verteidigen (Z. 412,

4 BoLLYwood als Positionierungsobjekt ist zwar eigentlich nicht als konkrete Serie einzustufen, allerdings wird das Genre von den Teilnehmenden wie eine einzige Serie behandelt (und später auch entsprechend in Frage gestellt, Z. 417-506), daher wurde entschieden, diesen Fall in die Kollektion zu integrieren. 
414, 432-434, 483, 487, 489-494). Die Bewertungen manifestieren sich sowohl in spöttisch anmutenden Inszenierungen wie Davids Tanzeinlagen (Z. 399, 447, 413) als auch der wiederholten Behauptung, die Filme würden keine - logische (Z. 482) oder verbalsprachlich realisierte (Z. 428) - Handlung beinhalten, sondern bestünden hauptsächlich aus tanzenden Darsteller*innen. Damit beanspruchen die Jungen epistemische Autorität zur Bewertung, die sich auf primär dieses Wissenselement bezieht. Als Auslöser für diese inhaltliche Fokussierung könnte Gisems Eingangsäußerung, sie hätte wie „die MEISten“ ,Bollywood-Tanzen“ anhand von YouTube-Videos gelernt (Z. 400-403), betrachtet werden. Darauf folgt eine Fremdpositionierung von Igdir und David, die sie scherzhaft zu einer Demonstration ihrer Tanzkünste auffordern (Z. 409-411). Gisem ignoriert die Fremdpositionierung und versucht stattdessen, ihre positive Bewertung mit dem stetigen Verweis auf ihre Faszination für die indische Kultur (Z. 408, 412, 504) zu begründen.

Schließlich spricht Gisem David seine Expertise explizit ab: „die TANzen nich so david; “(Z. 414), während sie seine Bewegungen imitiert. Daraufhin markiert David seinen $K^{-}$-Status deutlich (Z.415-416), wobei er sich mit der Kategorisierung „sowas“ (Z. 416) $)^{5}$ von dem Genre distanziert und gleichermaßen seine negative Bewertung aufrechterhält. Gisem indes sucht offenbar nach Möglichkeiten zur Vergemeinschaftung, indem sie die Gruppe fragt, ob sie „kein bollywood“ gucken (Z. 417). Mit Ali hätte sie einen potentiellen $K^{+}$-Vergemeinschaftungs-Kandidaten (Z. 420-421), der das Angebot jedoch nicht annimmt (Z. 430, 473). David, Igdir und Paul arbeiten schließlich konsequent daran, BoLlywood den Serienstatus und damit die Relevanz für diese Interaktion abzusprechen (Z. 422-431). Daher muss Gisem zunächst Aufwand betreiben, für das serielle Format von Bollywood $\mathrm{zu}$ argumentieren und es mit ihrer eigenen epistemischen Autorität, die sich in mehrfacher und kürzlicher Rezeption begründet, zu belegen (Z. 432-436).

Igdir positioniert Gisem einerseits als Expertin, indem er sie dazu auffordert, eine Szene aus einer Bollywood-Produktion zu rekonstruieren (Z. 449, 482). Andererseits stützt seine Rahmung, die Szene hätte keinen narrativen Sinn ergeben, die zuvor von den Kritikern angeführten Stereotype. Gisem nutzt die Aufforderung geschickt, um mittels der kontrastiven und hohe Gewissheit transportierenden Formulierung „aber es is halt INdien; n DORF; ist was ANderes“ (Z. 457-460) ihre positive Evaluation - die Faszination für die indische Kultur - erneut zu bekräftigen und den anderen impliziten $\mathrm{K}^{-}$-Status zuzuschreiben. Davids teasing-Versuch (Z. 461), ${ }^{6}$ indische und türkische Kultur gleichzusetzen, ignoriert sie. Zudem si-

5 Vgl. zu „sowas“ auch die Analysen in Abschnitt 7.3.3.

6 Eine ausführliche Analyse der Aushandlung zu türkischen Serien kann in Weiser-Zurmühlen (2020) nachgelesen werden. 
gnalisiert sie Widerstand gegen Igdirs Rahmung, indem sie die Un-Plausibilität der Handlung nicht auf deplatziertes Tanzen zurückführt, sondern auf die narrative visuelle Darstellung, dass ein Auto trotz Unfalls keinen sichtbaren Schaden davonträgt (Z. 478-479) (vgl. dazu auch Abschnitt 8.2.1). Tatsächlich wird ihre Argumentation und ihre $K^{+}$-Position erst dann anerkannt, als sie mit lauter Stimme und gestisch unterstützt sehr präzise Anzahl und Gelegenheiten für Tänze in Bollywood-Produktionen auflistet (Z. 484-498). Zumindest Igdir signalisiert Ratifizierung durch den Erkenntnisprozessmarker „ach so oke;“ (Z. 495). Davids anschließender Turn legt jedoch nahe, dass er mit einem Vergleich zwischen Bollywood- und amerikanischen Produktionen den Sonderstatus, den Gisem Bollywood-Serien zuschreibt, in Frage stellt (Z. 507). Damit beendet er zugleich die Sequenz und etabliert ein neues Gesprächsthema.

Gisem muss um die Anerkennung ihrer epistemischen Autorität zur positiven Bewertung kämpfen, da die Bewertungsmaßstäbe auf unterschiedlichen Ebenen angesiedelt sind. Die negativen Bewertungen der $K^{-}$-Teilnehmenden basieren auf pauschalen (Vor-)Urteilen, während Gisems Bewertung deutlich differenzierter ausfällt und sie sowohl mehr Gewissheit als auch Glaubwürdigkeit über ihre epistemische Haltung kommuniziert. Ihre $K^{+}$-Position wird jedoch nicht durchgängig anerkannt, sondern sie muss sie stetig aktualisieren. Auch in diesem Beispiel weicht niemand von der zuerst angezeigten evaluativen Haltung ab.

\subsubsection{Zusammenfassung: Die Praktik Absprechen von fremder epistemischer Autorität zur Bewertung bei $\mathrm{K}^{-}$}

Die Positionierungspraktik des Absprechens fremder epistemischer Autorität wird in Abbildung 7.3 schematisch visualisiert (siehe zur Erläuterung Abschnitt 6.4) und lässt sich folgendermaßen beschreiben:

- Strukturstelle(n): Mindestens zwei Gruppenmitglieder positionieren sich entgegengesetzt: Eine Person (A) positioniert sich als $\mathrm{K}^{+}$und bewertet die Serie positiv, eine Person (B) positioniert sich als $K^{-}$und bewertet die Serie negativ. Beide beanspruchen epistemische Autorität (eA) zur Bewertung der Serie.

- Ausgestaltung: Je nachdem, mit welcher Gewissheit die epistemische Haltung ausgestattet ist und auf welcher Grundlage die Bewertung durchgeführt wird, fällt die Praktik seitens der $\mathrm{K}^{+}$-Sprechenden unterschiedlich aus. Zunächst können die Teilnehmenden eine Brücke bauen und den $K^{-}$- Teilnehmenden die Gelegenheit geben, ihre evaluative Haltung zu relativieren. Im Anschluss an die Erkundigung nach dem tatsächlichen epistemischen Status der negativ Bewertenden - also nach der Glaubwürdigkeit der Wissensquelle - wird auf dieser Grundlage deren epistemische Autorität zur Bewertung in Frage gestellt. Daraufhin werden die unterschiedlichen Bewertungen - die sich meistens 
auf differierende Elemente der Serie beziehen - weiterhin konfrontativ und mittels der Ressource des Kontrastierens ausgehandelt. Es findet jedoch selten eine Änderung der evaluativen Haltungen der $K^{-}$-Teilnehmenden statt. Je subjektiver und weniger allgemeingültig die Bewertung formuliert ist, desto face-wahrender vollzieht sich die Sequenz.

- Interaktive Funktion(en): Die Praktik unterstützt die Selbstpositionierung als Expertin oder Experte und greift gleichzeitig bei drohendem face-Verlust. Sie soll dazu dienen, die eigene epistemische Autorität zur Bewertung zu sichern. Indem sie offensichtlich aufgrund mangelnden Wissens nicht berechtigten Personen abgesprochen wird, soll sichergestellt werden, die Deutungshoheit und finale Bewertung einer Serie zu sichern. Zum Umdenken und Einlenken werden die Negativbewertenden jedoch selten bewegt (Beispiel 20 und 21), es erfolgt höchstens eine minimale Ratifizierung des hohen epistemischen Status der $K^{+}$-Person (Beispiel 22).

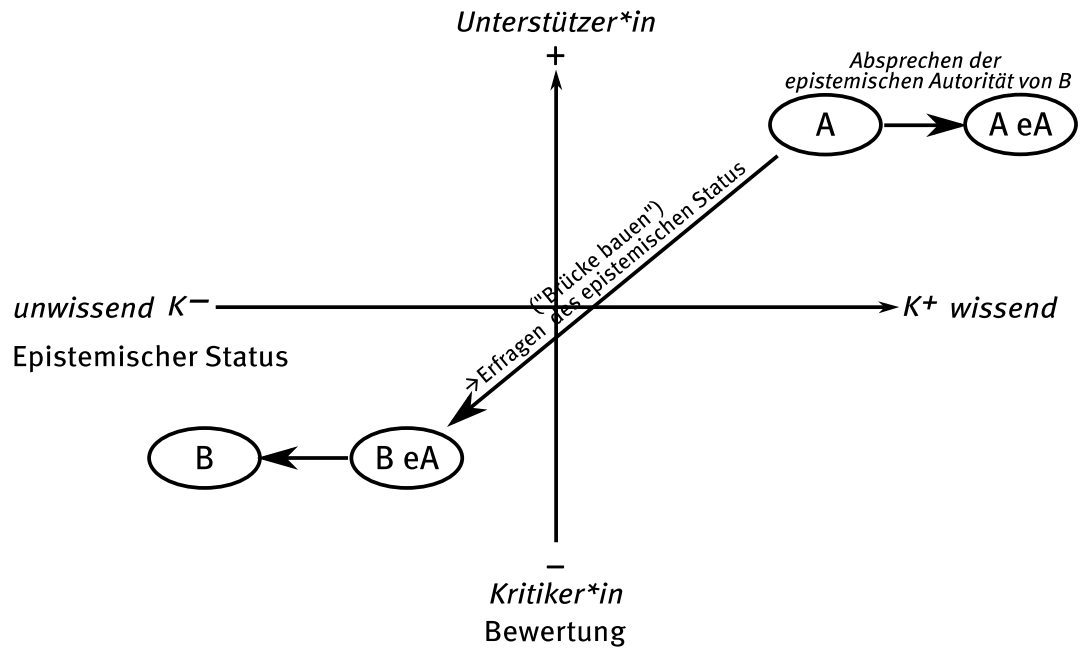

Abb. 7.3: Schematische Darstellung der Praktik Absprechen fremder epistemischer Autorität

Diese Praktik findet sich selten in den Daten. Aus genderbezogener Perspektive ist auffällig, dass es in allen Fällen männliche Teilnehmende sind, die trotz $K^{-}$- Status Expertise beanspruchen, und weibliche Teilnehmende, die ihnen die Autorität zur Bewertung wiederum absprechen. Auch die Selbst-Inszenierung in der Rolle des 
(sich im Nachhinein als unwissend herausstellenden) Kritikers (vgl. Abschnitt 6.4), die in den einzelnen Gesprächen insbesondere Johann für OST11 (Beispiel 21) und David für MNG13 (Beispiel 22) einnehmen, muss vor diesem Hintergrund betrachtet werden. Nichtsdestotrotz lässt sich auch feststellen, dass die Beteiligten Vergnügen aus dem Dissens ziehen und potentiell heikle und face-bedrohende Situationen schnell abwehren (für ausführliche Analysen zu gruppendynamischem Konsens und Dissens vgl. Abschnitt 7.3). Zu dieser Praktik greifen die Beteiligten somit erst verhältnismäßig spät.

\subsubsection{Empfehlen eines Rezeptionsmodus}

Auch diese Praktik basiert auf einer herunterstufenden Zweitbewertung, mit der die $\mathrm{K}^{+}$-Beteiligten jedoch anders als im Rahmen der Praktik Absprechen von fremder epistemischer Autorität umgehen. Sie erhöhen ihren eigenen epistemischen Status und zeigen - analog zu der Praktik Einbeziehen serienexterner Wissensquellen Wissen über eine Serie an, das über die Rezeption hinausgeht. Der Unterschied besteht darin, dass sie die anderen Teilnehmenden gezielt mit diesem Wissen ausstatten, um sie dahingehend zu belehren, wie sie die Serie mit dem erhaltenen Wissen ,besser' rezipieren können (vgl. zu Belehrungen v.a. Keppler $(1989,1994)$ sowie die Ausführungen in Abschnitt 2.2.3 und 4.3).

Die folgenden Fallbeispiele der Kollektion sind entlang dreier verschiedener Wissensbestände in Bezug auf Serien angeordnet. In Beispiel 23 wird notwendiges Kontextwissen als Verstehensvoraussetzung für die Serie empfohlen. Beispiel 24 beinhaltet eine Erläuterung zum Figurenverstehen und Beispiel 25 bezieht sich darauf, die symbolische Bedeutung einer Serie nachzuvollziehen. Leitend für die Beschreibungen dieser Praktik ist die Frage: Welches Wissen ziehen $\mathrm{K}^{+}$-Beteiligte wie heran, um $\mathrm{K}^{-}$-Beteiligten eine Anleitung zur Rezeption an die Hand zu geben, und welche interaktiven Konsequenzen ergeben sich daraus?

\subsubsection{CHARMED - Verfügen über notwendiges Kontextwissen}

(23) DIE serie muss man damit anfangen,

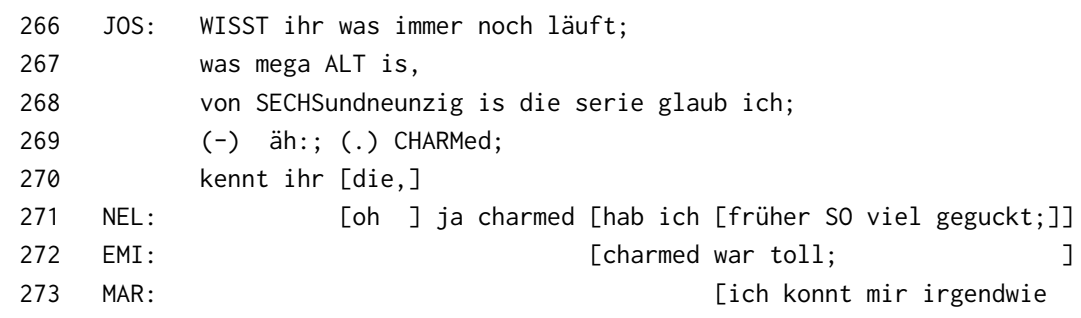


nich ANgucken;]

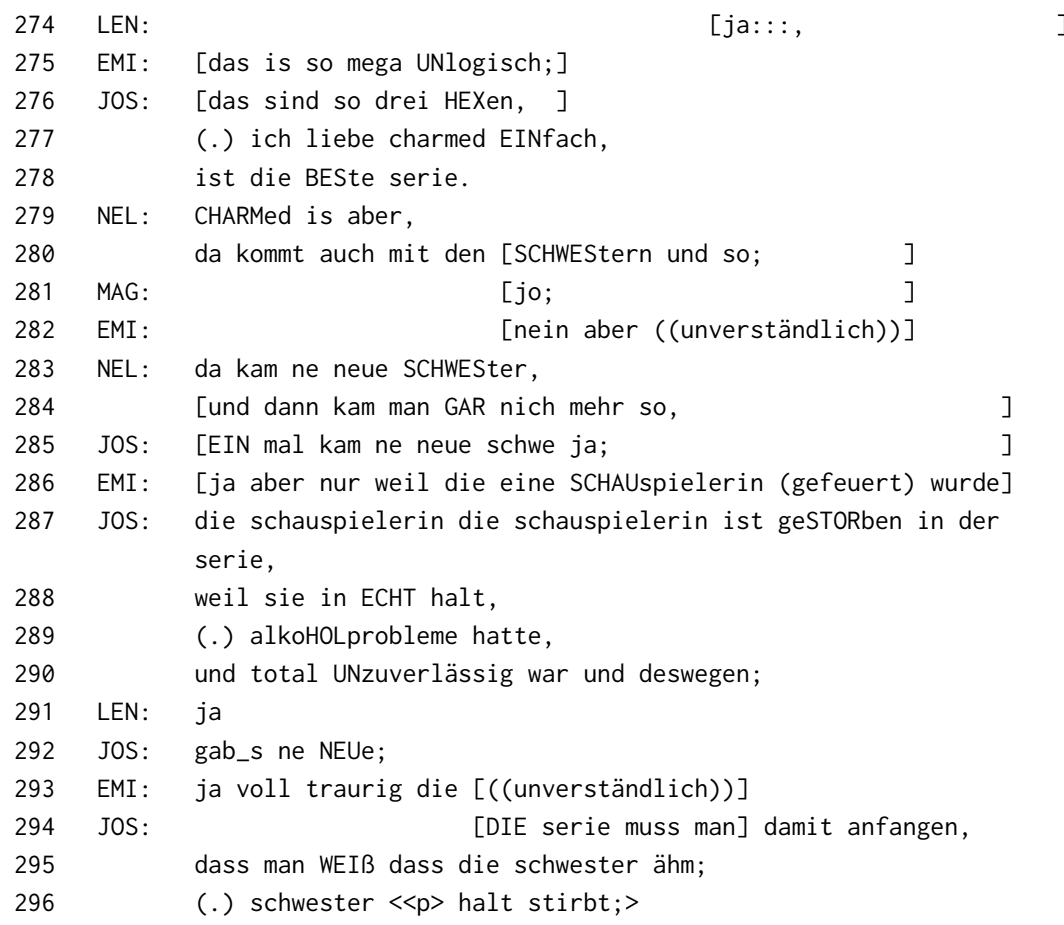

In dieser Sequenz geht es um die Serie CHARMED, die Josefine als sehr positiv bewertet und sich dazu als $K^{+}$positioniert (Z. 266-278). Nelli und Emilia markieren ebenfalls einen relativ hohen epistemischen Status und eine gute Bewertung, während Martha sich eher negativ äußert (Z. 271-273). Im Folgenden schwächen Emilia und Nelli jedoch ihre erste Bewertung ab, indem sie die Entwicklung der Serie kritisieren und ihr einen Qualitätsverlust aufgrund eines Figurenwechsels attestieren (Z. 275-286), für den Josefine abschließend eine Erklärung und darauf basierend eine Rezeptionsempfehlung formuliert (Z. 287-296).

Aufschlussreich für die Analyse der Praktik ist hier die genauere Betrachtung des interaktiven Kontextes in Bezug auf den Wechsel von Josefines epistemischer Haltung: Signalisiert sie zu Beginn eher oberflächliches Wissen, wie das Alter der Serie (Z. 267-268) oder die vage modalisierte Kategorisierung der Hauptfiguren als „so drei Hexen“ (Z. 276), verändert sie im Verlauf der Sequenz ihre epistemische Positionierung und zeigt einen höheren $K^{+}$-Status an, indem sie auf Kontextwissen verweist (Z. 287-292). Dieser Wechsel wird durch die vorangegangene Bewertungssequenz ausgelöst. Denn nachdem Josefine ihre positive Haltung der 
Serie gegenüber betont hat - mit der extreme case-Formulierung „beste Serie“ (Z. 278) -, schwächen Emilias und Nellis Bewertungen (Z. 275, 279-284) ihre erste Bewertung ab. Nellis abgebrochener Turn scheint sich darauf zu beziehen, dass sie nach dem Figurenwechsel der Handlung der Serie nicht mehr gut folgen konnte (Z. 284). Daraufhin signalisiert Emilia ebenfalls einen $K^{+}$-Status im Hinblick auf die damit zusammenhängenden Geschehnisse „hinter den Kulissen“, die den Charakterwechsel erklären, und die auf der Entlassung einer Schauspielerin beruhen (Z. 286).

Dieses Wissenselement greift Josefine auf und behandelt es im Folgenden als unzureichend (Z. 287). Dafür gibt sie über eine hohe epistemische Gewissheit detaillierteres Wissen über den Medienproduktionskontext preis: Die Schauspielerin habe Alkoholprobleme gehabt, sei unzuverlässig geworden und habe deswegen die Serie verlassen müssen (Z. 287-292). Indem Josefine ihre epistemische Haltung so gestaltet, dass sie Wissen anzeigt, das über die reine Serienrezeption hinausgeht - für diese Informationen ist eine Hintergrundrecherche notwendig -, beansprucht sie die epistemische Autorität zur Bewertung der Serie.

Ihre Expertise sichert sie nun abschließend durch eine Empfehlung. Sie instruiert die anwesenden Teilnehmenden sowie potentielle imaginierte Rezipient`innen in generalisierter Art und Weise, über welches Wissen sie verfügen sollten, bevor sie die Serie rezipieren (Z. 294-296). Damit erhöht sie die epistemische Glaubwürdigkeit ihrer epistemischen Haltung, um ihre Behauptung zu stützen. Zudem instrumentalisiert sie ihre Expertise dafür, andere Rezipient*innen dahingehend zu belehren, wie sie die Serie rezipieren sollten. Diese Belehrung in Form einer Empfehlung scheint durch die zweite negativere Bewertung ausgelöst worden zu sein.

\subsubsection{ARROW - Verstehen von Figurenverhalten}

(24) ihr müsst SO denken;

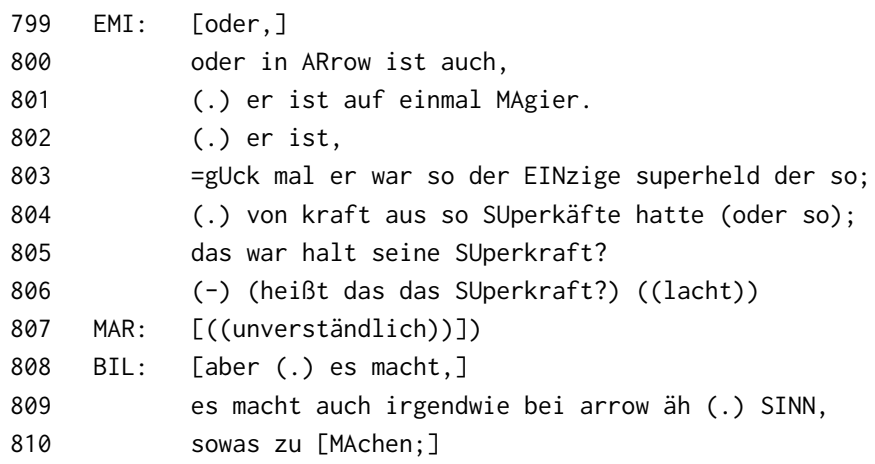




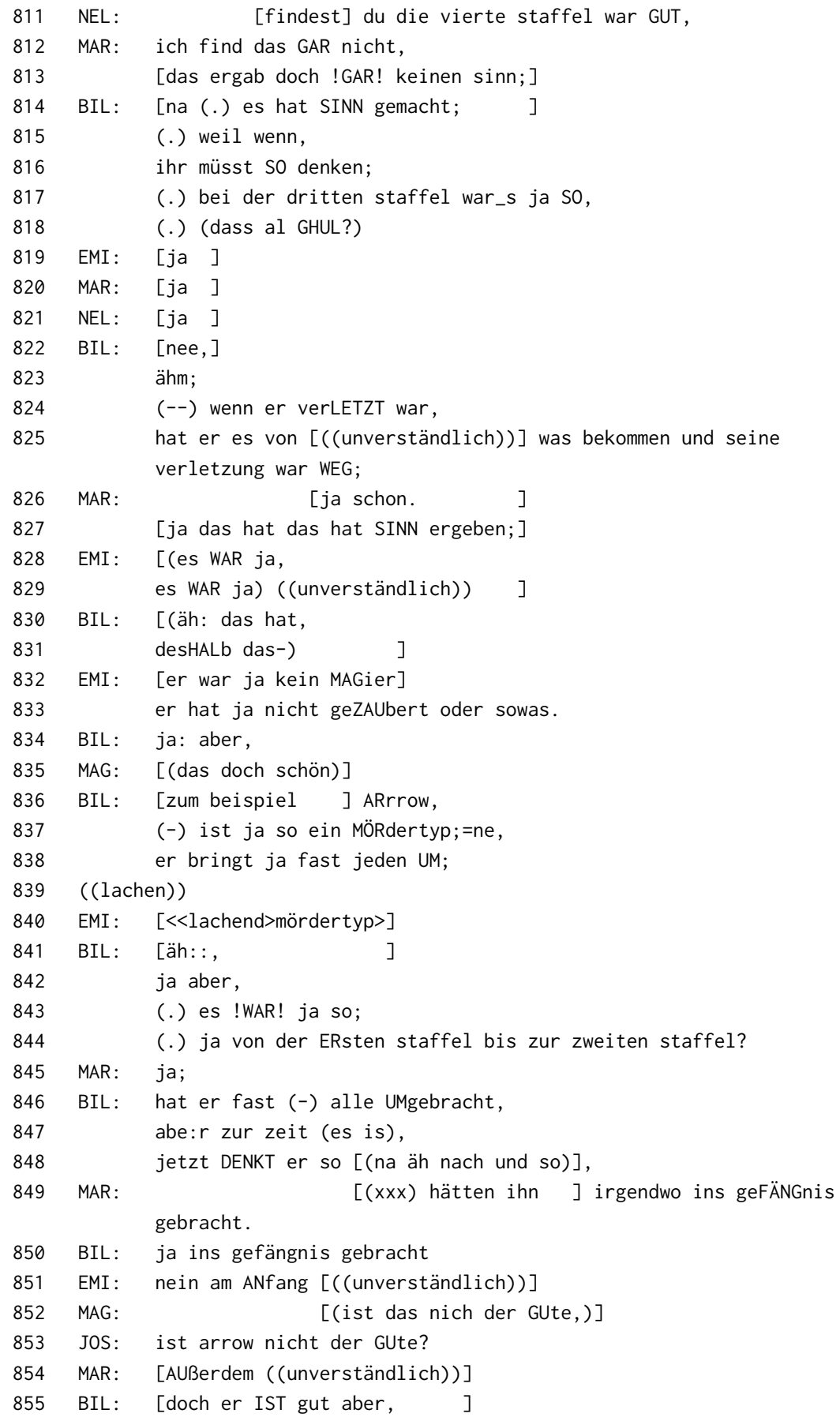




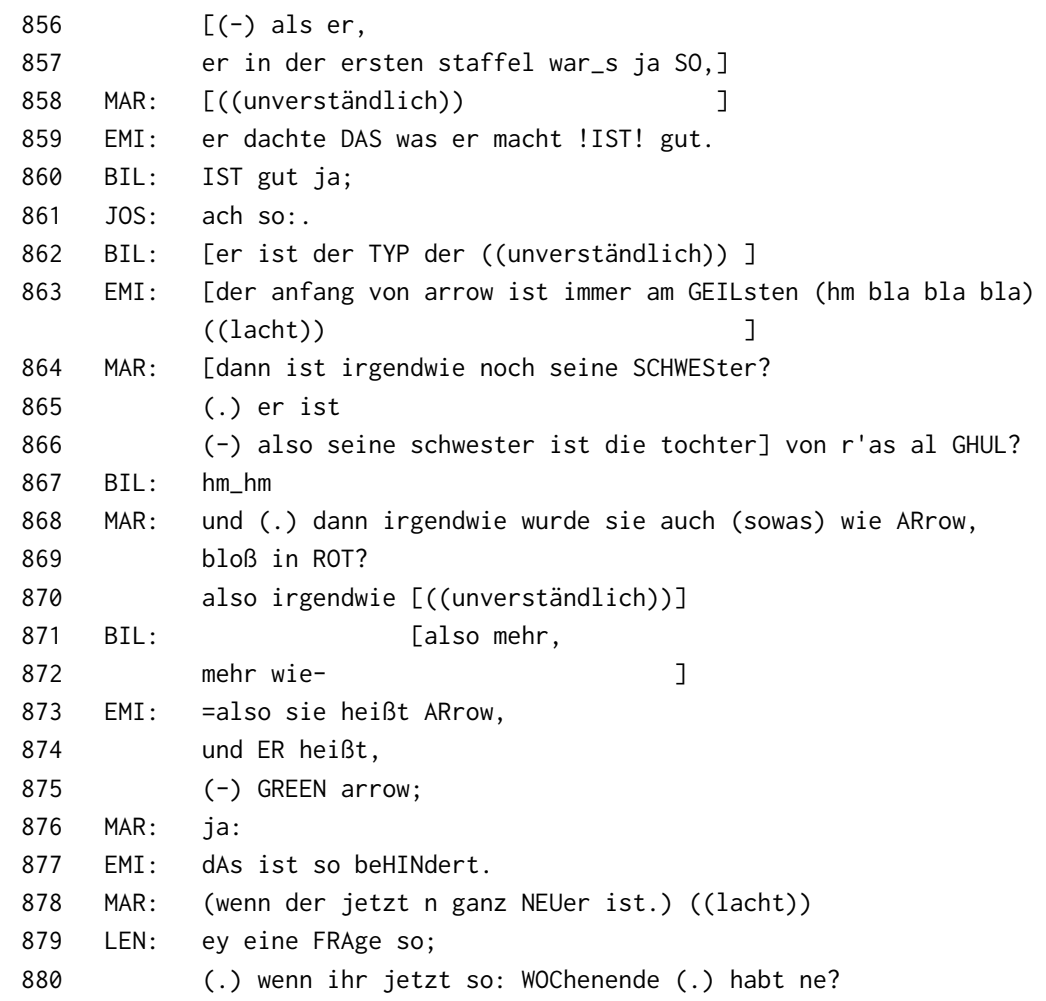

Eingebettet ist die Sequenz in die Frage nach unlogischen Handlungselementen in Serien (vgl. auch Abschnitt 8.2.1). Emilia bringt daraufhin das Gespräch auf die Serie ARRow und deren Hauptfigur, dessen Veränderungen im Laufe der Serie sie als nicht plausibel darstellt (Z. 799-806). Zu Beginn der Gruppendiskussion hatte Bilel sich bereits als Experte in Bezug auf die Serie positioniert, indem er sie als seine Lieblingsserie angegeben hatte. Er widerspricht Emilia und schreibt der Darstellung der Figur und deren Handeln Sinn zu. An der folgenden interaktiven Aushandlung beteiligen sich Emilia, Josefine und Martha (Z. 809-878).

Wie Josefine in Beispiel 23 verschiebt auch Bilel seine epistemische Haltung von eher oberflächlichen Informationen zu tiefergehendem Wissen. Hatte er zuvor lediglich den Inhalt der Serie - mit (unaufgeforderter) Unterstützung von Martha und Emilia - zusammengefasst (vgl. Anhang IV: MNG11, Z. 049-089), erläutert er nun in der hier vorliegenden Sequenz Gründe für die Entwicklung des Protagonisten. Auch dieser Wechsel wird durch die negativere Zweitbewertung ausgelöst: Als Emilia (erneut) gemeinsam mit Martha die Handlungslogik der Serie kritisiert 
(Z. 800-813), widerspricht Bilel der Bewertung der Teilnehmerinnen (Z. 814), indem er zunächst eine eher oberflächlich bleibende Erklärung basierend auf der Beziehung des Protagonisten zu seinem Gegenspieler R’as Al Ghul (Z. 818-825) anführt, die er mit der Aufforderung ,ihr müsst SO denken“ rahmt (Z. 816). Während Martha seine Erklärung ratifiziert (Z. 826-827), bezweifelt Emilia sie weiterhin (Z. 832-833). Daraufhin erläutert Bilel mittels der Ressourcen Kategorisieren, Kontrastieren und Rekonstruieren die narratologische Konzeption der Figur, die sich in ihrer moralischen Einstellung wandelt und mit dem Morden aufhört (Z. 836851). Marthas und Emilias Äußerungen finden parallel zu anderen Turns statt und sind daher schwer verständlich. Sie scheinen Bilels Ausführungen allerdings zu widersprechen, genau wie Magnus und Josefine, die seine Kategorisierung, Arrow sei ein „Mördertyp“ (Z. 837) in Frage stellen (Z. 852, 853). Ko-konstruktiv lösen daraufhin Emilia und Bilel den moralischen Widerspruch auf, indem sie auf die Beweggründe der Figur verweisen (Z. 859-860).

Analog zu Josefine in Beispiel 23 behandelt Bilel das Wissen der anderen Gruppenmitglieder als unzureichend. Mit der Formulierung „ihr müsst SO denken; “ empfiehlt er ihnen eine andere Art und Weise der Rezeption, mit der die Figur, ihr Handeln und ihre Entwicklung verstehbar und nachvollziehbar werden (sollen) (Z. 816, 847). Auch er beansprucht eine hohe Glaubwürdigkeit, weil er anzeigt, dass er die Serie auf einer anderen Ebene versteht. So sichert er sich mit dieser Praktik die Expertise und argumentiert für eine Serie, für die er aufgrund ihres Status als seine Lieblingsserie epistemische Autorität beansprucht.

\subsubsection{The WALKIng DeAd - Erkennen des narrativen Symbolgehalts} (25) the walking dead ist viel mehr in diesem, (-) HANdlungsschema;

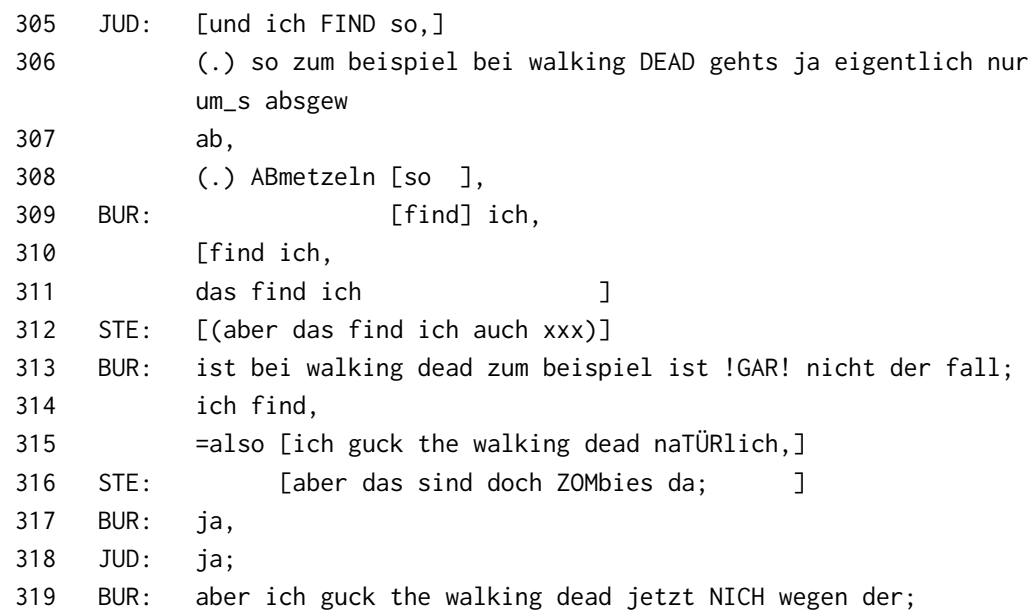




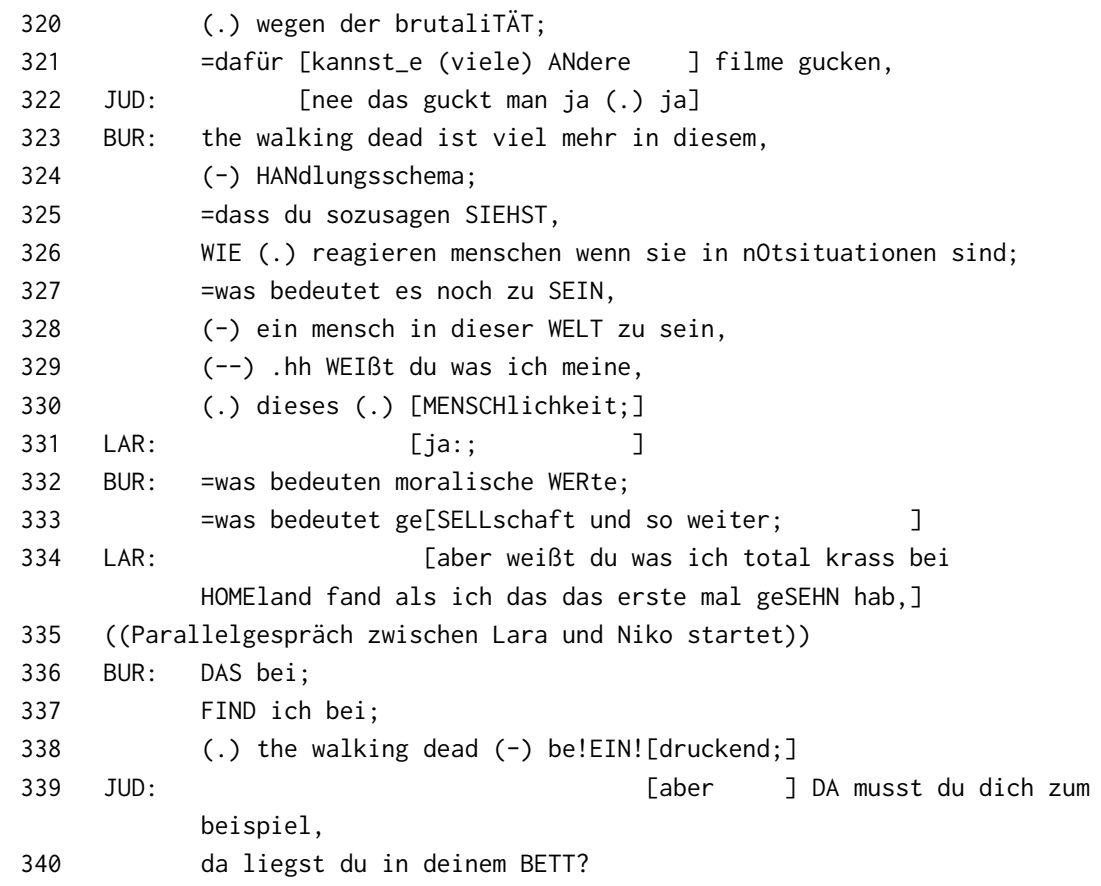

Burim wurde zuvor nach seinen Lieblingsserien gefragt und listet unter anderem THE WALKING DEAD auf. Mit der Formulierung „da hab ich ne ganze (.) ähm BANDbreite“ (vgl. EMS51, Z. 147 und Beispiel 50) signalisiert er einen hohen epistemischen Status in Bezug auf Serien generell und mit der expliziten Nennung von The WALKING DeAD als Lieblingsserie in Bezug auf diese Serie im Speziellen. Zudem bewertet er die Serie damit auch positiv. Etwa drei Minuten später beginnt die hier ausgewählte Sequenz, in der Judith erneut auf THE WALKING DEAD zu sprechen kommt. Sie zeigt minimales Wissen über die Serie und deren Thema an, wobei sie durch die Partikel „nur“ (Z. 306) und die Formulierung „ABmetzeln“ (Z. 308) eine negative evaluative Haltung anzeigt. Vergleichbar mit Beispielen 23 und 24 weist Burim im nächsten Schritt das von Judith angezeigte Wissen zurück. Er widerspricht ihr jedoch deutlich expliziter als Josefine Emilia in Beispiel 23 und markiert ihr angezeigtes Wissen mittels einer sehr starken Betonung, die hohe epistemische Gewissheit markiert, als falsch: „ist !GAR! nicht der fall;“ (Z. 313). Da Stephanie Alignment zu Judiths Aussage signalisiert und mit der Partikel „doch“ (Z. 316) epistemische Autorität beansprucht (vgl. dazu Analysen in Abschnitt 7.2.1), 
behandelt Burim beide Aussagen gleichzeitig als zwar auf inhaltlicher Ebene korrekt (Z. 317), aber dennoch als unzureichend.

So belehrt er die anderen Teilnehmenden - und potentielle, imaginierte Rezipient*innen - zunächst dahingehend, dass die Serie im Unterschied zu anderen Produktionen gerade nicht bloße Brutalität darstellen würde (Z. 319-321). Daran knüpft er seine Empfehlung eines Rezeptionsmodus an, d.h. er formuliert eine Instruktion, wie die Serie rezipiert und verstanden werden sollte, nämlich dass sie über die narrative Struktur hinaus eine symbolische Bedeutung aufweist und grundlegende anthropologische Fragen aufwirft (Z. 323-333). Damit schreibt auch er sich eine hohe Glaubwürdigkeit zu und wertet seinen epistemischen Status auf. So kann er eine höhere epistemische Autorität als Judith zur Bewertung der Qualität der Serie beanspruchen. Im Hinblick auf Belehrungen zeigt sich hier mit dem verhältnismäßig unaufwändigen Einstieg ein Unterschied zu Kepplers (1998) Ausführungen (vgl. auch Abschnitt 6.3.2). Daran zeigt sich, dass Belehrungen immer kontextsensitiv angepasst werden.

\subsubsection{Zusammenfassung: Die Praktik Empfehlen eines Rezeptionsmodus}

Die Positionierungspraktik des Empfehlens eines Rezeptionsmodus wird in Abbildung 7.4 schematisch visualisiert (siehe zur Erläuterung Abschnitt 6.4) und lässt sich folgendermaßen beschreiben:

- Strukturstelle(n): Eine Person (A) positioniert sich als $K^{+}$und bewertet die Serie positiv, bezeichnet sie häufig auch als Lieblingsserie. Diese Positionierung kann auch schon zu einem früheren Zeitpunkt stattgefunden haben. Eine andere Person (B) äußert eine herabgestufte, eher negative Bewertung der Serie, kommuniziert aber auch Wissenselemente, mit denen sie sich als $\mathrm{K}^{+}$positioniert, und beansprucht ggf. epistemische Autorität B (epA).

- Ausgestaltung: Auslöser ist die negative Bewertung eines Gruppenmitglieds in Kombination mit einem zuvor geäußerten, eher oberflächlichen Wissenselement. Dieses Wissenselement greift die sich als $\mathrm{K}^{+}$-positionierende Person auf und behandelt es entweder als unzureichend oder als nicht korrekt. Sie verschiebt ihre epistemische Haltung hin zu mehr Expertise, indem sie tiefergehendes Wissen anzeigt, mit dem sie zugleich signalisiert, dass sie es „besser weiß“ (vgl. auch Sidnell 2012 und Abschnitt 4.3) - sei es in Bezug auf Kontextwissen (Beispiel 23), Figurenverhalten (Beispiel 24) oder symbolische Interpretation (Beispiel 25). Anschließend sichert sie die epistemische Autorität, indem sie sich in eine hierarchiehöhere, epistemische Position begibt, die darin besteht, die anderen Gruppenmitglieder zu instruieren, die Serie in einer bestimmten Art und Weise zu rezipieren und sie damit ,besser‘ zu verstehen. 
- Interaktive Funktion(en): Die Praktik unterstützt die Selbstpositionierung als Expertin oder Experte, indem sie einerseits die eigene epistemische Autorität zur Bewertung sichert und sie anderen aufgrund unzureichenden Wissens nicht-berechtigten Teilnehmenden abspricht. Andererseits führt sie auch dazu, dass Expertise über die Vermittlung tieferen und gesicherteren Wissens über die (Lieblings-)Serie hergestellt wird.

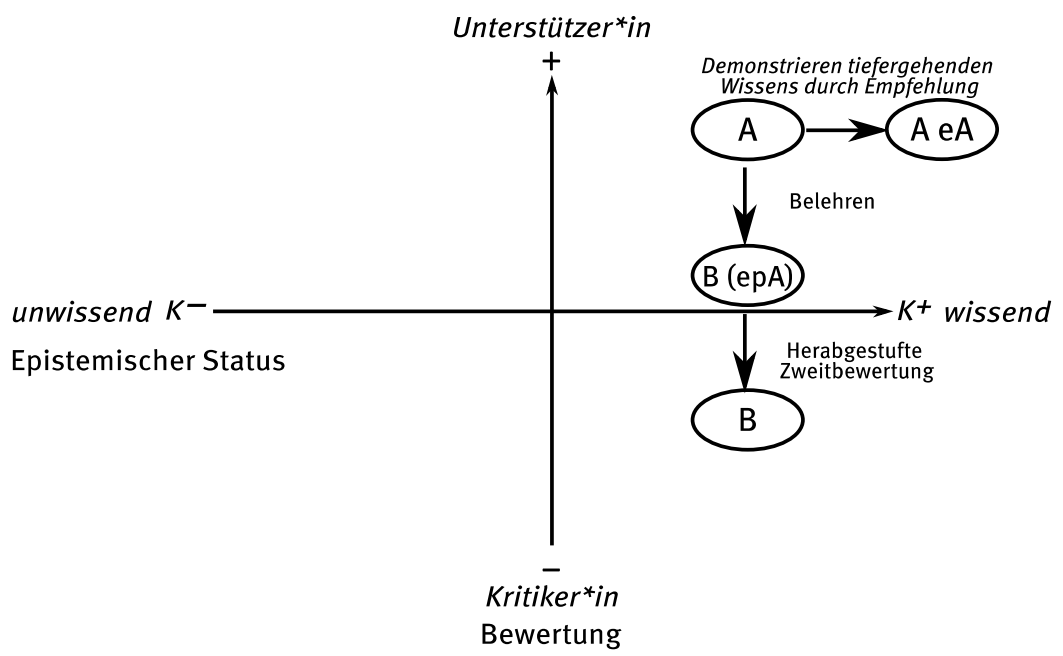

Abb. 7.4: Schematische Darstellung der Praktik Empfehlen eines Rezeptionsmodus

Diese Praktik wird insbesondere bei Lieblingsserien und als sehr positiv bewerteten Serien verwendet. Dabei steht weniger die Vergemeinschaftung und Vergewisserung, das Gleiche zu mögen, im Vordergrund, sondern eher das Werben für die eigene Serie. Auch mit dieser Praktik werden Wissensasymmetrien ausgeglichen (vergleichbar mit der Praktik des Einbeziehens serienexterner Wissensquellen, Abschnitt 7.1.2), allerdings nicht als primäre Informationsübermittlung, sondern mit dem Ziel der Belehrung (vgl. Keppler 1989), d.h. die Teilnehmenden sollen etwas mit den ihnen mitgeteilten Wissenselementen „machen“. 


\subsubsection{Zusammenfassung: Positionierungspraktiken zum Hochstufen des epistemischen Status bei $K^{+}$}

In diesem Abschnitt wurden die vier Praktiken beschrieben:

(1) Managen divergenter Wissensbestände

(2) Einbeziehen serienexterner Wissensquellen

(3) Absprechen von fremder epistemischer Autorität zur Bewertung einer Serie

(4) Empfehlen eines Rezeptionsmodus

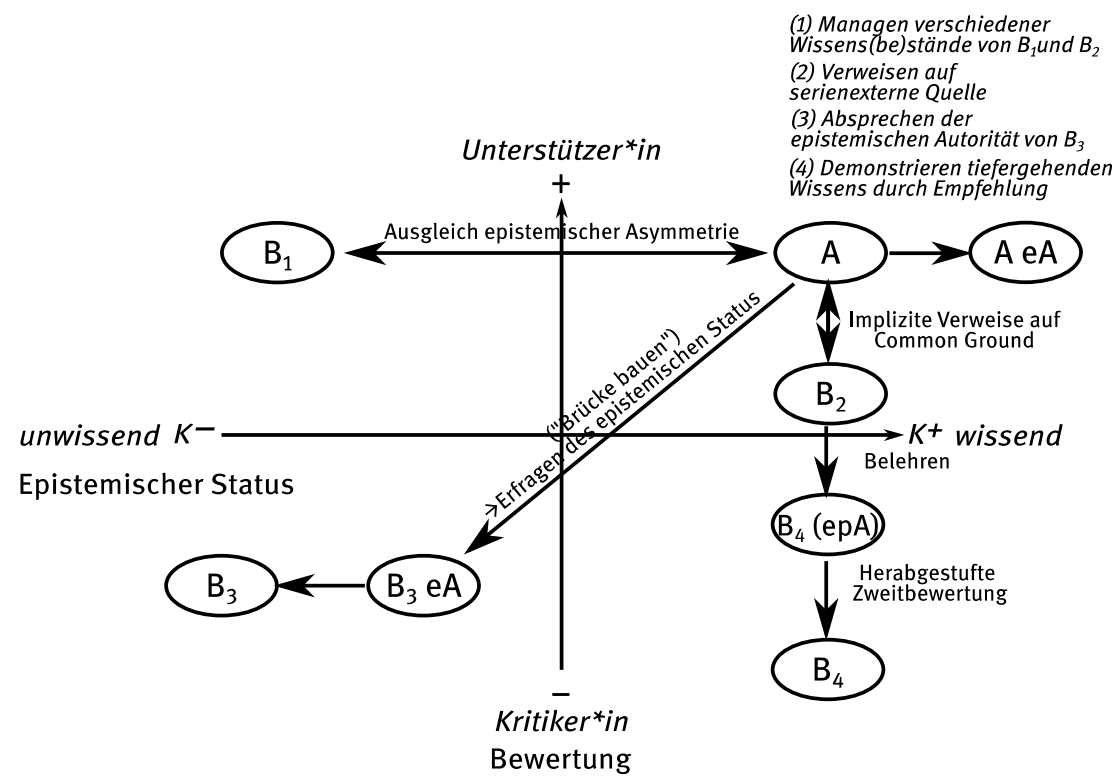

Abb. 7.5: Schematische Darstellung der rekonstruierten Positionierungspraktiken, um epistemische Autorität bei einem $K^{+}$-Status zu beanspruchen.

Diese Praktiken beziehen sich auf eine/n oder mehrere $K^{+}$-Sprecher*innen, die epistemische Autorität zur Bewertung einer Serie beanspruchen. Dafür stufen sie im Laufe einer Sequenz über ihre epistemische Haltung, die ausreichende Gewissheit und Glaubwürdigkeit vermittelt, ihren epistemischen Status hoch. Voraussetzung dafür ist, dass sie einen interaktiv als ausreichend behandelten epistemischen Zugang zu der Serie anzeigen. In Abbildung 7.5 ist schematisch abgebildet, wie die Praktiken dieser Personen (hier als A angegeben) auf den interaktiven 
Kontext innerhalb einer Sequenz abgestimmt sind, d.h. je nachdem wie sich die anderen Teilnehmenden (hier repräsentiert durch $B_{1-4}$ ) positionieren.

Bei vergleichbaren oder neutralen Bewertungen, aber unterschiedlichen Wissensständen und Wissensbeständen der anderen Teilnehmenden, managen sie diese Unterschiede. Sie gleichen epistemische Asymmetrie zwischen ihnen und den $\mathrm{K}^{-}$-Beteiligten aus und verweisen zugleich auf geteiltes Wissen mit den $\mathrm{K}^{+}$Beteiligten. Indem ihnen auch die Expertise dafür zugeschrieben und bestätigt wird, können sie die epistemische Autorität zur Bewertung einer Serie beanspruchen. Ihre Glaubwürdigkeit können sie erhöhen, wenn sie serienexterne Wissensquellen einbeziehen. Dadurch können Wissensasymmetrien ausgeglichen werden, allerdings ist der Interaktivitätsgrad geringer und die Personen B haben weniger Möglichkeiten zur Partizipation über unterstützende Gesprächsarbeit wie Nachfragen o.Ä. hinaus. Wenn Interagierende A mit Teilnehmenden konfrontiert werden, die sich als unwissende Kritiker`innen positionieren und ihnen damit den Anspruch auf epistemische Autorität streitig machen, können sie über das Erhöhen ihrer eigenen epistemischen Gewissheit und Glaubwürdigkeit eine Fremdpositionierung gegenüber B vornehmen. Indem sie B auf den zu geringen epistemischen Zugang hinweisen, sprechen sie ihnen zugleich die epistemische Gewissheit und Glaubwürdigkeit und damit die epistemische Autorität zur Bewertung ab. Positionieren sich Personen B dagegen als wissend mit einer herabgestuften Zweitbewertung, können Teilnehmende A eine Belehrung anschließen, um den eigenen epistemischen Status weiter hochzustufen. Indem Personen A tiefergehendes Wissen signalisieren, das Wissen von B als unzureichend markieren sowie B erklären, wie sie das transferierte Wissen verstehen sollten - ihnen also eine Empfehlung aussprechen -, erhöhen sie die eigene Glaubwürdigkeit, aber auch Gewissheit und können somit epistemische Autorität beanspruchen.

Selbstverständlich werden damit immer nur Momentaufnahmen erfasst und die Expertise einer Person muss immer interaktiv bestätigt und aufrechterhalten werden. So kann im nächsten Moment oder in der nächsten Sequenz die Verteilung auf dem Feld wieder anders aussehen. Zudem handelt es sich hier um eine analytische Trennung, so können die Praktiken auch gebündelt (als „Praktikengefüge“; vgl. Androutsopoulos 2016: 341) vorkommen: Wenn Burim in Beispiel 25 Judith über die ,korrekte“ Rezeptionsart belehrt, spricht er ihr damit zugleich auch die epistemische Autorität für ihre Bewertung ab und managt sowohl ihren als auch Stephanies divergierende Wissensstände. Die Einordnung erfolgt hier aus zweierlei Gründen: Zum einen verändert Burim seinen eigenen epistemischen Status von eher oberflächlichem Wissen zu tieferem Wissen und zum anderen instruiert er die anderen Gruppenmitglieder bezüglich der Einordnung dieses Wissens. Auch Friederikes Ausführungen in Beispiel 19 zu THE WIRE könnte als Empfehlung ei- 
nes Rezeptionsmodus - der auf der beruflich bedingten Expertise einer externen Person beruht - interpretiert werden.

Als Ziele von Positionierungspraktiken als Expertinnen, die einen hohen epistemischen Status in Bezug auf eine konkrete Serie anzeigen, lassen sich insgesamt festhalten, dass die Teilnehmenden Partizipation anstreben und ermöglichen, Wissensasymmetrien ausgleichen, sich mit anderen vergemeinschaften und Allianzen bilden, eigene Expertise beanspruchen und verteidigen und letztlich den eigenen Geschmack kommunizieren und ggf. dafür argumentieren.

\subsection{Positionierungspraktiken zum Hochstufen des epistemischen Status bei $K^{-}$}

In diesem Abschnitt werden die folgenden vier Praktiken beschrieben:

(1) Anzeigen von partiellem Wissen

(2) Berufen auf die Bewertung anderer

(3) Kommunikativ erfolgreiches bzw. erfolgloses Etablieren eines Positionierungsobjekts

(4) Nachträgliches Revidieren des epistemischen Status

Bei diesen Praktiken liegt der Fokus auf denjenigen Teilnehmenden, die im Laufe der Sequenz einen $K^{-}$-Status im Hinblick auf eine Serie kommunizieren, d.h. einen geringen epistemischen Zugang anzeigen. Es wird herausgearbeitet, welche Praktiken sie nutzen, um über die Gewissheit und Glaubwürdigkeit ihrer epistemischen Haltung ihren Status graduell hochzustufen. Das primäre Ziel der Nutzung dieser Praktiken besteht darin, an der aktuellen Diskussion - insbesondere an Bewertungsaktivitäten - partizipieren zu können. Da diese Praktiken weniger komplex sind als die unter Abschnitt 7.1 diskutierten, werden sie unter Rückgriff auf verschiedene Sequenzen des Korpus stärker zusammenfassend dargestellt. Lediglich in Abschnitt 7.2.4 werden zwei Fallbeispiele detaillierter analysiert. Die zitierten Transkriptausschnitte können entweder im Anhang oder - falls vorhanden - an anderer Stelle in der Arbeit über den nachgestellten Verweis auf die Beispiel-Nummer und Seite nachgelesen werden. Die Abschnitte sind folgendermaßen strukturiert: Nach einer kurzen Einführung in die Praktik erfolgt eine Beschreibung der sequenziellen Umgebungen, in die sie kontextuell eingebettet sein kann. Ausgehend davon werden basierend auf mikroanalytischen Sequenzanalysen die Ausgestaltung der Praktik und ihre interaktiven Konsequenzen und Funktionen beleuchtet, bevor abschließend mögliche interaktive Funktionen diskutiert werden. 


\subsection{1 "geht_s da eigentlich nur um TOD und so, “ - Anzeigen von partiellem Wissen}

Mit partiellem Wissen sind jene Wissenselemente gemeint, die über die epistemische Haltung entweder von der äußernden Person selbst oder von anderen als (zu) oberflächlich und mit wenig epistemischer Gewissheit und/oder Glaubwürdigkeit versehen behandelt werden. Die Praktik, partielles Wissen anzuzeigen, findet sich in den vorliegenden Daten selten an sequenzinitiierender Position und ist überwiegend als Folgepositionierung kontextualisiert. Hauptsächlich wird sie dafür genutzt, um trotz geringem epistemischen Zugangs zu der Serie am Gespräch partizipieren und den Gruppenprozess aufrecht erhalten zu können.

Eine Möglichkeit, partielles Wissen anzuzeigen, besteht darin, im Rahmen einer gerade stattfindenden Bewertungsinteraktion die Serie ebenfalls kurz zu kommentieren und damit einen gewissen Grad an epistemischer Gewissheit zu kommunizieren. So nimmt beispielsweise Nelli an der Bewertungsinteraktion zu der Serie UNDER THE Dome teil, obwohl sie zuvor mittels Kopfschütteln auf Josefines Frage, wer die Serie kennt, einen $K^{-}$-Status markiert hatte (vgl. Anhang IV: MNG11, Z. 739). Als die Gruppe sich wenig später darüber amüsiert, dass die in der Serie aufgestellten Regeln offenbar nicht für alle Figuren gelten (Z. 763-775), schließt sie sich der Scherzinteraktion an: „TSCHÜSS; (-) oh hier ist ne TÜR; (.) mensch; (Z. 776-778). Damit inszeniert sie kurz ein fiktives Szenario, das die von Magnus bemängelte fehlende Logik der Serie pointiert (Z. 777-778). Die Äußerung unterstützt sie gestisch, indem sie aus der Figurenperspektive heraus eine imaginäre Tür öffnet und mit der Stimme der Figur spricht. Ihre Inszenierung führt genau wie Magnus' Äußerungen zuvor - zu Gelächter der Gruppe (Z. 778). Diese Praktik setzt Nelli öfter ein, so auch in Beispiel 15, als sie auf Josefines empörend inszenierte Bewertung zur verpassten Kuss-Gelegenheit einer Figur aus THE ORIGINALS lakonisch mit „wie im ECHten leben“ (Z. 336) reagiert. Sie partizipiert damit zwar nur minimal am Gesprächsgeschehen, erntet aber bisweilen zumindest eine kurze Ratifizierung (Z. 778).

Eine weitere Möglichkeit ist es, nicht nur im Rahmen der gegenwärtigen Sequenz zu kommentieren, sondern auch Rückbezüge zu vorherigen Diskussionen zu ziehen. So spielt beispielsweise Pauls Äußerung „das is FAST wie die frau da im bettlaken" (vgl. Anhang V: MNG13, Z. 750) als Kommentar zu Davids Rekonstruktion einer Szene aus der Serie QuANTICo (Z. 698-749) auf die Scherzinteraktion der Gruppe zu einer Episode aus ICERDE einige Minuten zuvor an (vgl. MNG13, Z. 590-608). Die Kommentare zeigen vor allem an, dass die Sprechenden aktiv am Geschehen teilnehmen, sie erheben jedoch nicht den Anspruch auf längere (Bewertungs-)Turns oder das längerfristige Rederecht. In Anlehnung an Baldauf und Klemm (1997) zum fernsehbegleitenden Sprechen zeigen sich teilweise Par- 
allelen zwischen dieser Praktik und der von den Autor*innen so bezeichneten „Häppchen-Kommunikation“ (vgl. Abschnitt 2.2.2).

Interagierende mit $\mathrm{K}^{-}$-Status können allerdings durchaus ein höheres Maß an epistemischer Gewissheit kommunizieren, indem sie sprachlich unterschiedlich gestaltete Fragen an die $\mathrm{K}^{+}$-Beteiligten formulieren: W-Fragen oder Fragen mit Verberststellung signalisieren eine weniger hohe Gewissheit als negative V1- oder tag-Fragen (vgl. auch die Tabelle von Raymond und Heritage 2006 in Abschnitt 4.3). So zeigt Jan in Beispiel 19 nach Friederikes missverständlicher Zuschreibung einer $\mathrm{K}^{+}$-Position in Bezug auf die Serie THE WIRE in Form von drei Fragen jeweils unterschiedliche Grade epistemischer Gewissheit an. Die erste Nachfrage zum Inhalt der Serie „ist das irgendwie so: ist das nich IRgendwie mit ä::hm:, m:edikaMENten, = also es sind irgendwie DROgen oder sowas? (-) nich,“ (Z. 436-441) ist ein länger konstruierter Turn, der Reparaturen, vage Modalisierungen, Pausen und Verzögerungssignale aufweist. Die verhältnismäßig lange Pause (Z. 441) legt nahe, dass die Zielrichtung der Frage als dispräferiert markiert wird. Tatsächlich behandelt Friederike sein angezeigtes Wissen im Folgenden auch als nur teilweise korrekt. Die nächste Frage formuliert Jan somit vorsichtiger. Er überlässt Friederike die epistemische Autorität zu deren Beantwortung und beansprucht selbst nur Wissen bezüglich der Kategorisierung des Serientyps (vgl. auch Beispiel 10): „ist ist das so ne case of the WEEK (.) folge, =also se serie also wo: in jeder folge sozusagen ein neuer FALL dran kommt?" (Z. 464-465) und sichert mit seiner abschließenden Frage „oke aber es ist jetzt nicht so wie MONK oder so dass da jed, in jeder folge so_n-“ (Z. 470-471) Verständnis. Letztere Äußerung lässt wiederum mehr epistemische Gewissheit sowie Kenntnis über die Serie MoNK, die er dem Serientyp case of the week zuordnet, vermuten. Nach wechselseitiger Bestätigung ist die Sequenz damit face-schonend beendet und Jan hat zu sowohl seiner eigenen als auch Friederikes Partizipation am Gespräch erfolgreich beigetragen.

Auch andere Interagierende, die diese Praktik verwenden, zeigen damit an, dass sie über minimales Wissen verfügen. Besonders häufig tritt dabei die Formulierung geht es (nicht) um X? auf, wie zum Beispiel Josefines Äußerung „geht_s nich um TIEre?“ (vgl. Beispiel 14) zeigt. Auch Sonja, die zu der Serie RIVERDALE zuvor geäußert hatte, dass ihre Geschwister, aber nicht sie selbst die Serie schauen (vgl. Anhang IX: OST11, Z. 643, 646), adressiert Verena, die die Serie offensichtlich kennt, mit der Frage „geht_s da eigentlich nur um TOD und so,“ (Z. 658). Dieses mit durchaus hoher Gewissheit kommunizierte Wissenselement weist Verena zurück und kontrastiert es mit der Kategorisierung „es geht um BITCHfight;“(Z. 661).

Am meisten stufen die Teilnehmenden ihren epistemischen Status allerdings über Äußerungen oder Tag-Questions mit der Partikel „doch“ (vgl. z.B. Hentschel 1986) hoch. In den vorliegenden Daten wird „doch“ häufig widersprechend genutzt, allerdings - ganz im Sinne der Praktik des partiellen Wissenanzeigens - ohne An- 
spruch auf längeres Rederecht. Deutlich wird das in der Aushandlungssequenz um Bollywood-Produktionen (vgl. Beispiel 22), in der mehrere Teilnehmer Gisems Argumentation, dass darin nicht ausschließlich getanzt würde, ignorieren. Als die Gruppe diskutiert, ob BoLlywood auch eine Serie im Sinne der Gesprächsaufgabe ist, wendet sich Paul mit der Formulierung ,da sprechen die doch quasi nur WEnig;=ne, die tanzen halt (nur)“ (vgl. MNG13, Z. 428, 431) an die anderen und versucht mit dieser zwar minimalen, aber als gewiss markierten WissenselementeAnzeige, Gisems epistemische Autorität zu untergraben. Auch Stephanies Äußerung in Bezug auf die Serie THE WALKING DeAD „aber das sind doch ZOMbies da;““ (vgl. EMS51 Z. 316, Beispiel 25) beansprucht, ausreichend Wissen zu haben, um Burim widersprechen zu können. Seine Belehrung wird damit kurzzeitig mit dem Verweis auf den angenommenen common ground als unbegründet behandelt.

Die primäre interaktive Funktion, die mit dieser Praktik bedient wird, ist die, Wissensäußerungen trotz $K^{-}$-Position zum Zweck der Bewertung tätigen zu können und damit - mehr oder weniger kooperative (Abschnitt 6.1.2) - Gesprächsarbeit zu leisten. Die Interagierenden signalisieren damit einander, dass sie weiterhin aktive Partizipierende sind und arbeiten an den kommunikativen Aufgaben des Positionierungsobjekts mit. Wenn die Teilnehmenden partielles Wissen in Bezug auf eine Serie anzeigen, die ihnen eigentlich nicht gut bekannt ist, können sie auch auf abstrakteres, serienbezogenes Wissen zurückgreifen (vgl. dafür Analysen in Kapitel 8); insbesondere mittels der Produktion einer Äußerung wie ,das ist (doch) in jeder Serie so" (vgl. dazu Abschnitt 8.3). Zudem kann die Praktik dazu funktionalisiert werden, sich entweder in Übereinstimmung mit anderen Gruppenmitgliedern zu positionieren, ihnen zuzustimmen und sich damit zu vergemeinschaften oder auch ihnen in Form von Fragen, die Zustimmung einladen, zu widersprechen und sich damit voneinander abzugrenzen.

\subsection{2 „es soll richtig gut sein?““- Berufen auf die Bewertung anderer}

Für die Praktik des Anzeigens partiellen Wissens ist ein gewisser epistemischer Zugang zu der Serie die Voraussetzung, so dass evaluative Kommentierungen oder Fragen möglich sind. Bei nur minimaler oder gar keiner Kenntnis einer Serie kann eine Bewertung allerdings nicht auf eigener Erfahrung beruhen, d.h. die epistemische Haltung kann wenig Glaubwürdigkeit transportieren. Eine Praktik, um dieser Schwierigkeit zu begegnen, ist das Berufen auf die Bewertung abwesender Dritter. Im Goffman'schen (1981: 144) Sinne nehmen die Sprechenden damit die Rolle des „animators“ an, die qua Redewiedergabe nicht ihre eigene Bewertung, sondern die anderer angeben. 
Die kontextuelle Einbettung kann unterschiedlich ausfallen: So ist es beispielsweise möglich, dass Beteiligte als explizit $K^{-}$fremdpositioniert (,ihr guckt game of thrones nich oder, “vgl. Anhang IV: MNG11, Z. 411) werden und damit indirekt in Zugzwang gebracht werden, sich evaluativ zu äußern (vgl. Anhang IV: MNG11, Z. 411-427). Andersherum können auch $K^{-}$-Beteiligte die Sequenz initiieren, indem sie unter Berufung auf Urteile Dritter von denjenigen Gruppenmitgliedern, die die Serie (gut) kennen, eine Bewertung einfordern (vgl. Anhang I: EMS51, Z. 995-1035). Eine weitere Möglichkeit ist die, dass die Bewertung über Dritte als zustimmende oder ablehnende Zweitbewertung innerhalb einer evaluativen Sequenz eingebracht wird (vgl. Anhang II: EMS53, Z. 133-143).

Die interaktive Ausgestaltung der Praktik hängt eng mit der kontextuellen Umgebung zusammen. Mit Äußerungen wie „aber es soll GUT sein; ich will das AUCH bald anfangen.“ (MNG11, Z. 418-419) oder „alle SAgen es soll richtig gut sein?“ (Z. 422) passen Leni und Bilel sukzessive ihre Bewertung an die positive Grundstimmung der Gruppe an und signalisieren ihre Bereitschaft, die Serie GAME of THRONEs ebenfalls zu rezipieren. Die $K^{+}$-Beteiligten würdigen diese Aussagen, indem sie die animierten Bewertungen hochstufen und begründen (Z. 416, 421, 425427), so dass die in dieser Art und Weise durchgeführte Praktik unproblematisch und als Reaktion präferiert zu sein scheint. Stephanie, die als Sequenzinitiierung von den anderen eine Einschätzung der Serie BETTER CALL SAUL einfordert, gibt zunächst erforderliches Kontextwissen (EMS51, Z. 1002-1026), ohne eine (animierte) Bewertung preiszugeben (d.h. sie überlässt die erste Positionierung den anderen, vgl. Abschnitt 6.5.3). Als die eingeforderten Bewertungen negativ ausfallen (Z. 1025, 1030), formuliert sie die Bewertung Dritter als Widerspruch: „also ICH höre bis jetzt immer die soll gUt sein.“ (Z. 1032). Die Sequenz wird schlichtend (das ist ja geSCHMACKSsache“, Z. 1034) abgeschlossen. Alex signalisiert mit seiner Zweitbewertung zu BREAKING BAD ,ich hab mal gehört dass die dritte auch toTA:L, mit nem HÄNger versehen,“ (EMS53, Z. 137, 139) Alignment zu der vorherigen Bewertungsinteraktion, in der die Qualität der Serie über die Staffeln hinweg als abnehmend beurteilt wurde. Diese Äußerung wird allerdings als dispräferiert behandelt, indem ihm die $K^{+}$-Beteiligten solange widersprechen (Z. 140, 142), bis er im Rahmen von Reparaturen die Gewissheit seiner epistemischen Haltung wieder relativiert (Z. 143).

Vergleichbar mit der Praktik des Einbeziehens serienexterner Wissensquellen kann auf gesprächsexterne Bewertungen verwiesen werden, um sich selbst in Relation dazu (epistemisch) zu positionieren und der epistemischen Haltung mehr Glaubwürdigkeit zu verleihen. Auch wenn die Bewertung als nicht die eigene gerahmt wird, kann sie dennoch interaktiv als solche behandelt werden. Sie kann hochgestuft oder es kann ihr widersprochen werden. Eine simple Zustimmung, wie sie zu solchen Bewertungen geäußert werden kann, für die die Sprecherin oder der 
Sprecher selbst verantwortlich ist, lässt sich in den Daten jedoch nicht beobachten. Primäres interaktives Ziel der Praktik ist auch hier wieder, über das Hochstufen des epistemischen Status an der Interaktion zu partizipieren. So können Bewertungen auch mit wenig Wissen platziert und mit einer zukünftigen Rezeptionsabsicht kombiniert werden. Zudem ist es dadurch möglich, die Bewertung aus der externen Quelle mit den Einschätzungen der Gruppe zu vergleichen. Dementsprechend stellt Kontrastieren (aktueller epistemischer Status mit zukünftigem Rezeptionsvorhaben; hear-say-Bewertung mit Gruppenbewertung usw.) eine zentrale Ressource für diese Praktik dar. Bei zustimmenden Bewertungen stellt sich diese Praktik als unproblematisch heraus. Interaktiv heikel wird es bei stark voneinander abweichenden Bewertungen, da sodann von den $\mathrm{K}^{+}$-Beteiligten die epistemische Autorität zur Bewertung abgesprochen werden kann (vgl. Abschnitt 7.1.3), so dass Reparatursequenzen initiiert werden müssen.

\subsection{3 „ganz KURZ; (.) by the way, “ - Kommunikativ erfolgreiches bzw. erfolgloses Etablieren eines neuen Positionierungsobjekts}

Eine weitere Praktik, die sich bei $K^{-}$-Teilnehmenden beobachten lässt, die wenig epistemischen Zugang zu der diskutierten Serie signalisieren, ist es, einen Themenwechsel zu initiieren. Dafür schließen sie entweder eine inhaltlich zum aktuellen Interaktionsgeschehen oder strukturell zu einer Äußerung eines Gruppenmitglieds passend kontextualisierte ,Assoziation“ (im Sinne einer second story; vgl. Jefferson 1978, vgl. zur Funktion von second stories im Kontext von stance taking auch Siromaa 2012) an und versuchen damit, ein neues Positionierungsobjekt zu etablieren (vgl. zur Erklärung der sequenziellen Strukturierung Abschnitt 6.5.3). Meistens findet diese Praktik dann Verwendung, wenn Teilnehmende über einen bestimmten - in der Länge variierenden - Zeitraum hinweg keinen verbalen Beitrag oder nur minimale (Rezeptions)signale zum Gespräch beigetragen haben (vgl. etwa die Analyse zu Marthas abnehmender Partizipation in Beispiel 9). Das ist unter anderem der Fall in Beispiel 14. Josefine zeigt einen geringen epistemischen Status in Bezug auf die Serie THE 100 an, indem sie sich nur mit partiellem Wissen daran beteiligt (MNG11, Z. 557, 595-596; vgl. auch Analysen in Abschnitt 7.2.1). Schließlich signalisieren sowohl Emilias sinkende Intonationskontur (Z. 624) als auch Lenis Erkenntnisprozessmarker „ach so oKE;“ (Z.625), ein potentielles Ende der Sequenz. Josefine ergreift die strukturelle Gelegenheit und etabliert erfolgreich die Serie Lost (vgl. Anhang IV: MNG11, Z. 626-629) als nächstes Positionierungsobjekt.

Die kontextuelle Einbettung der Praktik besteht also vorrangig darin, an einem TRP (transition relevant place; vgl. Levinson (1990: 296)) eine neue Sequenz zu initiieren. Wenn allerdings kein ${ }^{\star}$ e weitere ${ }^{\star}$ r Beteiligte ${ }^{\star}$ r interaktiv die Sequenzi- 
nitiierung bestätigt, hat die Praktik kommunikativ keinen Erfolg. Beispielsweise versucht Jan in Beispiel 20 mit der Äußerung ,ich finde alarm für kobra ELF klasse; “ (RHG10, Z. 866), ein neues Positionierungsobjekt zu etablieren. Er schließt damit strukturell passend an Leons vorherige Aussage an, er hielte MoRD MIT AUSSICHT für die einzige gute deutsche Serie, die er kennt (Z. 865), so dass ALARM FÜR KOBRA 11 also Jan zufolge indirekt ebenfalls als gute deutsche Serie kategorisiert würde. Seine Äußerung überlappt jedoch mit Katharinas positiver Bewertung (Z. 867), die dazu führt, dass Mitglieder der Gruppe sich weiterhin über MORD MIT AUSSICHT vergemeinschaften (vgl. Analysen in Abschnitt 7.1.1). ALARM FÜR KOBRA 11 hat also keine Chance mehr, in dieser Sequenz Gesprächsthema zu werden. Das Gleiche geschieht mit Katharinas Bewertung ,ich find türkisch für ANfänger noch gut“ (vgl. Beispiel 19; RHG10, Z. 422), die sie parallel zu Friederikes Äußerung produziert (Z. 420, 424). Theoretisch platziert sie ihren Turn trotz kurzer Überlappung (Z. 420) an einem TRP, allerdings beansprucht Friederike mittels Selbstwahl weiterhin das Rederecht zu THE WIRE und TÜRKISCH FÜR ANFÄNGER wird erst zu einem späteren Zeitpunkt diskutiert (vgl. Z. 646 und Analysen in Abschnitt 7.3.3).

Die Ausgestaltung der Praktik ist also wenig komplex. Meistens besteht die Äußerung nur aus einer Präferenzäußerung (Beispiel 19 und 20) oder der Frage nach dem epistemischen Status der anderen Teilnehmenden (Beispiel 14, S. 126) bezüglich des neuen Positionierungsobjekts. Tatsächlich ist Josefine aus der Gruppe MNG11 mit dieser Praktik im Rahmen der vorliegenden Daten am ,erfolgreichsten' in dem Sinne, dass das von ihr initiierte Objekt auch tatsächlich nächstes Gesprächsthema wird. $\mathrm{Zu}$ einem anderen Zeitpunkt unterbricht sie beispielsweise Martha mit der Formulierung „ganz KURZ; (.) BY the way, (.) habt ihr alle mary POPpins gesehen?" (vgl. Anhang IV: MNG11, Z. 1091-1093). Damit rahmt sie zum einen den Themenwechsel als Nebensequenz und bereitet zum anderen eine anschließende Belehrung vor, indem sie fragt, ob den anderen bekannt sei, dass die Schauspielerin ebenfalls in dem Film PLÖTZLICH PRINZESSIN mitspielen würde (Z. 1096, 1099). Auch wenn ihr die Belehrung nur teilweise gelingt, da ihre als ,neu markierte Information einzelnen Gruppenmitgliedern schon bekannt ist (Z. 1110) und einzelne Personen daher dispräferiert reagieren -, kann sie erfolgreich eine Sequenz zu MARY PopPINs etablieren. Jan dagegen, der mit seiner Etablierung von ALARM FÜR KOBRA 11 nicht erfolgreich war, steht nun unter dem Zugzwang, sich ebenfalls zu MORD MIT AussichT zu positionieren, was zu Aushandlungen der epistemischen Autorität zur Bewertung führt (vgl. Analyse zu Beispiel 20, S. 148).

Das Etablieren eines neuen Positionierungsobjekts kann auch teilweise erfolgreich sein, indem es von einem anderen Gruppenmitglied minimal ratifiziert, dann jedoch nicht weiter bearbeitet wird. Eine solche Realisierungsform der Praktik findet sich in den Daten der Gruppe OST11. Während Johann über die Serie ADVENTURE TIME berichtet und sie bewertet (vgl. Anhang IX: OST11, Z. 116-137), versucht 
Sevcan, mit Bob DER BAUMeISTER eine neue Sequenz zu initiieren, die strukturell an eine zuvor geäußerte Bewertung von ADVENTURE TIME anschließt.7 Zunächst hat Sevcan keinen kommunikativen Erfolg, da epistemische Aushandlungen folgen (Z. 132-136), so dass sie sich ebenfalls wieder an der Hauptinteraktion beteiligt. Wenige Sekunden später jedoch ratifiziert Robert parallel zu anderen Turns Sevcans Aussage, indem er eine Zweitbewertung anschließt, die ihre Äußerung abschwächt: „aber nur der !AL!te bob der baumeister; der neue ist KACke“ (Z. 139, 142). Seine Bewertung nimmt niemand aus der Gruppe - auch Sevcan nicht - auf, so dass es bei dieser kurzen Paarsequenz bleibt.

Teilnehmende nutzen diese Praktik, um bei eigenem $\mathrm{K}^{-}$-Status wieder am Gesprächsgeschehen partizipieren zu können und eine Serie als Positionierungsobjekt zu etablieren, zu der sie selbst eine $\mathrm{K}^{+}$-Position zeigen und somit die Möglichkeit haben, selbst epistemische Autorität zu beanspruchen (z.B mit einer der in Abschnitt 7.1 beschriebenen Praktiken). Die Praktik fällt unterschiedlich erfolgreich aus. Zum einen ist die sequenzielle Platzierung wichtig, d.h. es ist relevant, ob die Etablierung eines neuen Positionierungsobjekts an einer strukturell passenden Stelle geschieht, also ob die Initiierung angemessen kontextualisiert ist. Überlappungen scheinen dafür eher hinderlich zu sein, müssen es aber nicht. Außerdem scheinen sich hier auch gruppenbezogene Unterschiede zu manifestieren: In der Gruppe RHG10 funktioniert das Etablieren von neuen Positionierungsobjekten weniger gut, während die Teilnehmenden noch damit beschäftigt sind, eine Serie zu diskutieren und sich gegenseitig noch nicht das Ende einer Sequenz angezeigt haben. Eine Erklärung dafür könnte darin liegen, dass sich diese Gruppe zu Beginn der Gruppendiskussion gesprächsorganisatorisch darauf geeinigt hat, der Reihe nach ihre Lieblingsserien zu nennen und jeweils einzeln zu diskutieren. Gruppe MNG11 signalisiert im Unterschied dazu Offenheit für Serien-Nennungen zu jeder Zeit von jeder Person, so dass Themen leichter initiiert, aber auch wieder abgebrochen werden können (vgl. auch Abschnitt 6.1.1).

\subsection{4 „steht auf meiner LISte drauf;“ - Nachträgliches Revidieren des epistemischen Status}

Kennzeichnend für die bisher beschriebenen Praktiken ist, dass der $K^{-}$-Status den anderen Gruppenmitgliedern implizit oder explizit kommuniziert wurde, d.h. dass epistemische Haltung und epistemischer Status übereinstimmen - wie es Heritage

\footnotetext{
7 Allerdings legt die prosodische Gestaltung dieser Bewertung nahe, dass Johanns Äußerung ,ich !HAS!se die sendung“ (Z. 127) eine Zurückweisung von Roberts Unterstellung, er sei der einzige, der diese Serie möge, darstellt.
} 
(2012: 7f) zufolge überwiegend der Fall ist. Er erläutert aber auch, dass es in der Interaktion zu Abweichungen kommen kann: „However, while the principle of epistemic congruency is often realized in fact, this realization is far from inevitable. Epistemic status can be dissembled by persons who deploy epistemic stance to appear more, or less, knowledgeable than they really are“ (Heritage 2012: 7). Die folgenden beiden - für das vorliegende Korpus eher untypischen - Fallbeispiele stellen Sequenzen dar, in denen epistemische Haltung und epistemischer Status einer Person für einen gewissen Zeitraum inkongruent sind, d.h. dass die eigentliche $K^{-}$-Position erst nachträglich kommuniziert wird. Zuvor wurde mittels belehrender Rekonstruktionen eine $K^{+}$-Position beansprucht, indem die jeweiligen Teilnehmenden Sophie (Beispiel 26) und Tim (Beispiel 27) sich der Praktik des Managens divergenter Wissens(be)stände bedienen.

(26) steht auf meiner LISte drauf;

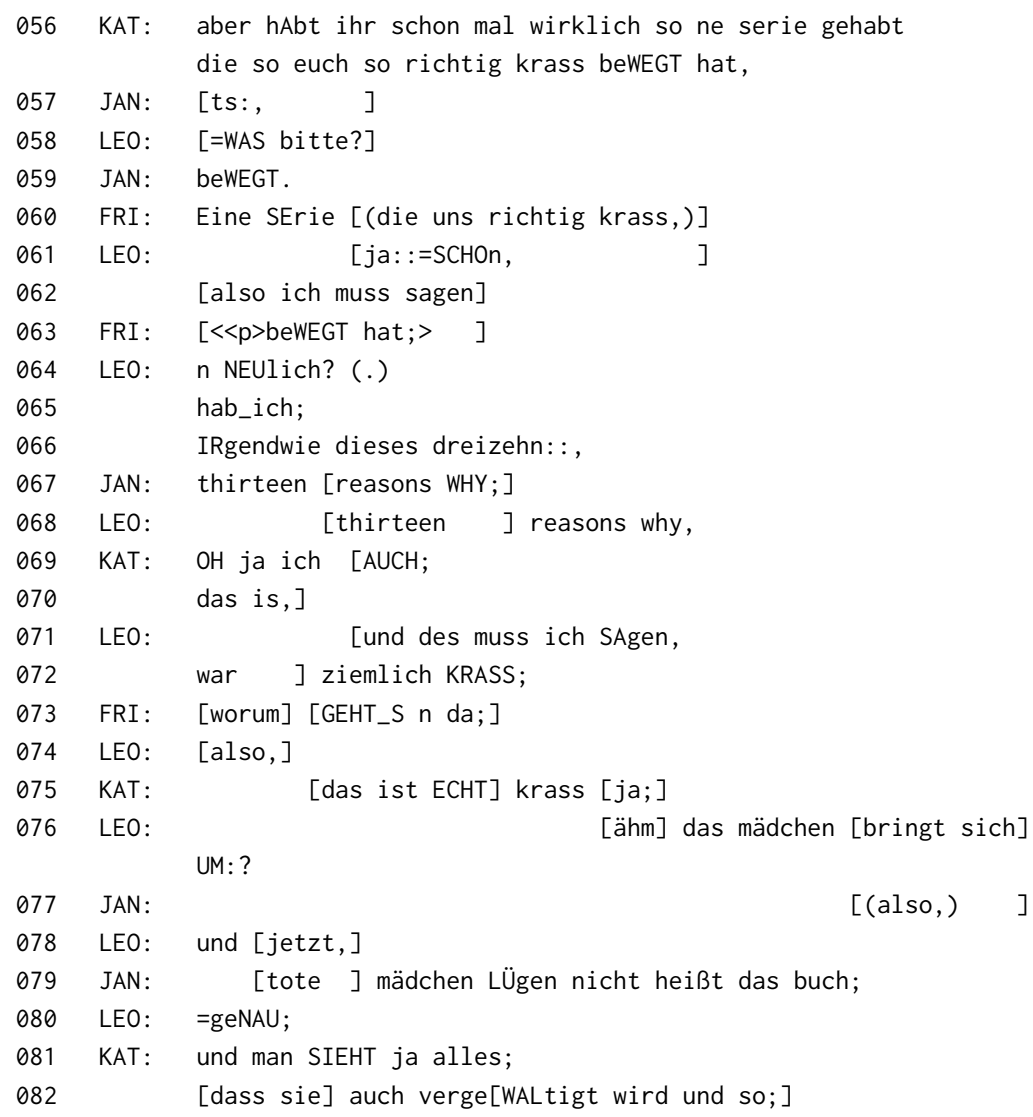




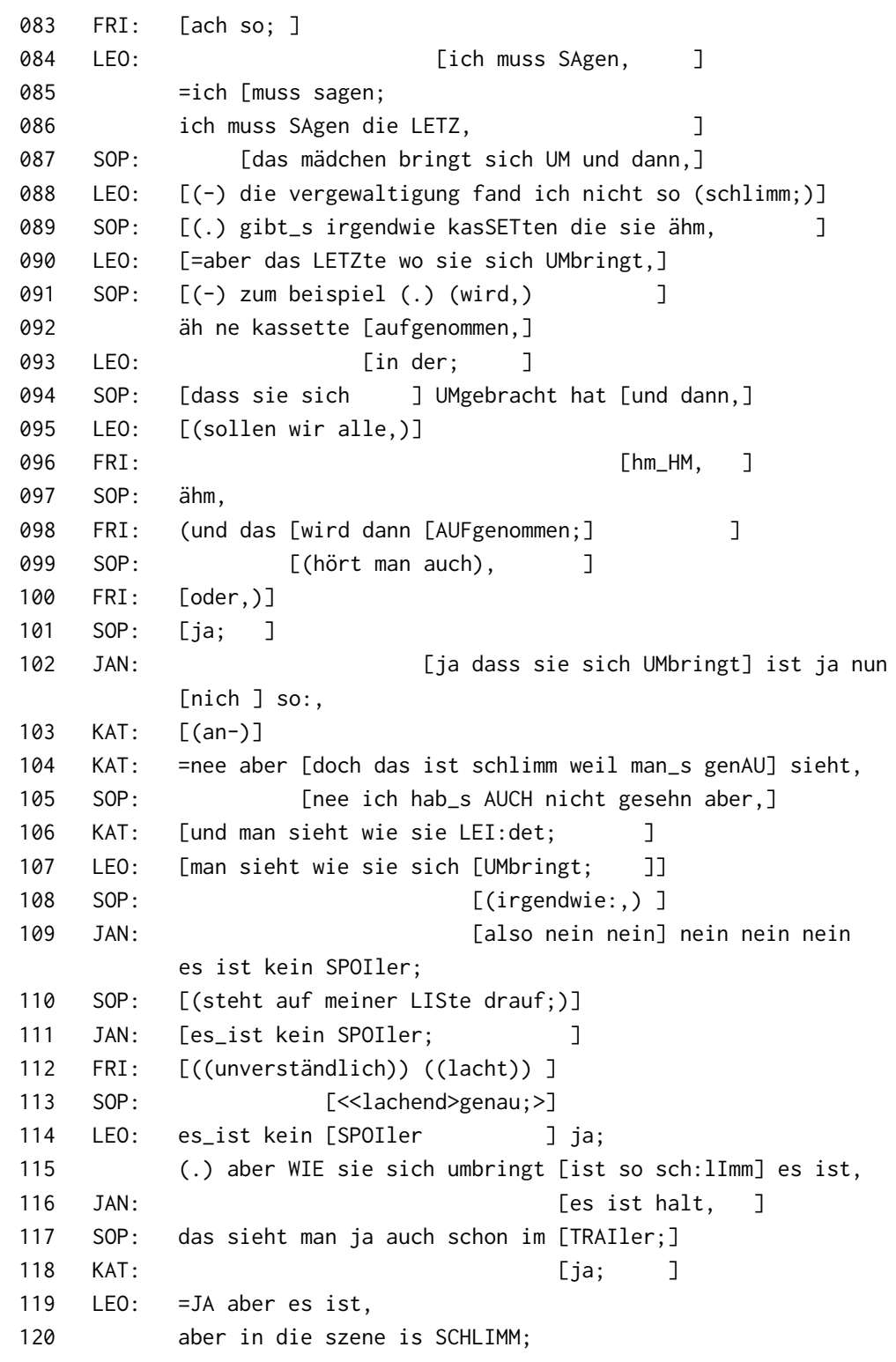

RHG10, 1:53-2:34

Die Gruppe diskutiert die Serie 13 ReAsons Why, nachdem Katharina von den anderen wissen möchte, ob sie eine bestimmte Serie schon mal „richtig krass bewegt“ habe (Z. 056). Hier wird auf der Rezeptionsebene auf emotionales Involvement 
Bezug genommen (vgl. dazu sowohl Abschnitt 2.2.1 als auch Abschnitt 8.2.4.1). Der Fokus der Analyse wird im Folgenden auf den Veränderungen von Sophies epistemischer Positionierung im Laufe der Sequenz liegen.

Katharina ratifiziert Leons Kategorisierung der Serie als eine, die ihn "krass bewegt hat" (Z. 069, 075) und signalisiert damit ebenfalls einen hohen epistemischen Status in Bezug auf diese Serie. Sophie äußert sich an an dieser Stelle nicht. Nachdem Friederike eine Rekonstruktion zur Behebung epistemischer Asymmetrie einfordert (Z. 073), beginnt eine ko-konstruktive Elaborierung des Plots, an der sich sowohl Leon (Z. 076, 078) und Katharina (Z. 081-082) als auch Jan (Z. 077, 079) beteiligen. Die Informationen, die Friederike angeboten werden, beschränken sich dabei auf zentrale Elemente der Handlung (Suizid, Z. 076 und Vergewaltigung, Z. 082) sowie auf den Hinweis, dass es ein äquivalentes deutschsprachiges Buch mit dem Titel Tote MÄDCHEN LÜGEN NICHT (Z. 079) gibt. Katharina und Leon bearbeiten schließlich gemeinsam die ursprüngliche Frage nach dem emotionalen Erleben der Serie auf der Ebene der visuellen ästhetischen Darstellung (Z. 081, 084-086, 088, 090, 093).

Während Leon Katharina hinsichtlich der Frage, welche Szene am schlimmsten dargestellt wird, widerspricht (Z. 088), bearbeitet Sophie parallel dazu weiterhin im Rahmen einer Nebenkommunikation Friederikes Frage nach dem Inhalt der Serie (Z. 087, 089, 091-092, 094), die sie um ein weiteres Handlungselement (Dokumentationszweck der Kassetten; Z. 091-092) erweitert. Friederike ratifiziert Sophies Rekonstruktion und signalisiert ihr Verstehen durch eine bestätigungseinladende Nachfrage (Z. 098), die Sophie wiederum bejaht (Z. 101). Mit dieser Rekonstruktion zeigt Sophie einen hohen epistemischen Status an. Sie stattet ihre epistemische Haltung sowohl mit hoher Gewissheit als auch mit Glaubwürdigkeit aus und positioniert sich so als diejenige, die über die epistemische Autorität für eine Rekonstruktion verfügt - etwas, das in den meisten Fällen Personen vorbehalten ist, die eine Serie (gut) kennen (vgl. Analysen in Abschnitt 7.1.1).

Leon und Katharina handeln nach wie vor aus, welche Szenen in der Serie wie detailliert gezeigt werden (Z. 102-104, 106-107). In der Interaktion zwischen Sophie und Friederike geschieht nun parallel Folgendes: Sophie revidiert ihren zuvor signalisierten hohen epistemischen Status, indem sie an Friederike gerichtet äußert: ,nee ich hab_s AUCH nicht gesehen aber, steht auf meiner LISte drauf; “ (Z. 105, 110). Damit verändert sie ihre Position von $\mathrm{K}^{+} \mathrm{zu} \mathrm{K}^{-}$. Dieser Wechsel wird in Abbildung 7.6 visuell durch einen Pfeil dargestellt. Mit der Formulierung ,steht auf meiner LISte drauf "verweist sie auf die von den meisten Streaming-Plattformen angebotene Option, Serien und Filmtitel digital vorzumerken, um sie zu einem späteren Zeitpunkt anzuschauen (vgl. auch Abschnitt 2.1.2). Diese nachträgliche Änderung ihres epistemischen Status wird interaktiv unproblematisch behandelt. Friederike teilt Sophie etwas - leider aufgrund mehrerer überlappender Äuße- 
rungen - Unverständliches mit und lacht (Z. 112), was Sophie ebenfalls lachend ratifiziert (Z. 113).

Letztlich setzt sich sequenziell die Diskussion von Katharina und Leon durch, indem Leon abschließend den interaktiven Raum erhält, anhand der Suizid-Szene aus der Serie zu erläutern, warum ihn diese Serie „krass bewegt hat“ (Z. 118-124, 150-204). Ein weiteres Mal beansprucht Sophie Expertise, indem sie Jans SpoilerDisclaimer im Hinblick auf den Suizid der Protagonistin (Z. 109, 114) bestätigt. Sie merkt an, dass dieser Teil der Handlung bereits im Trailer gezeigt wird (Z. 117). Damit referiert sie erneut auf die Quelle ihres Wissens (den Trailer der Serie), die sie zuvor als Begründung für die Rekonstruktion des Plots genutzt hat.

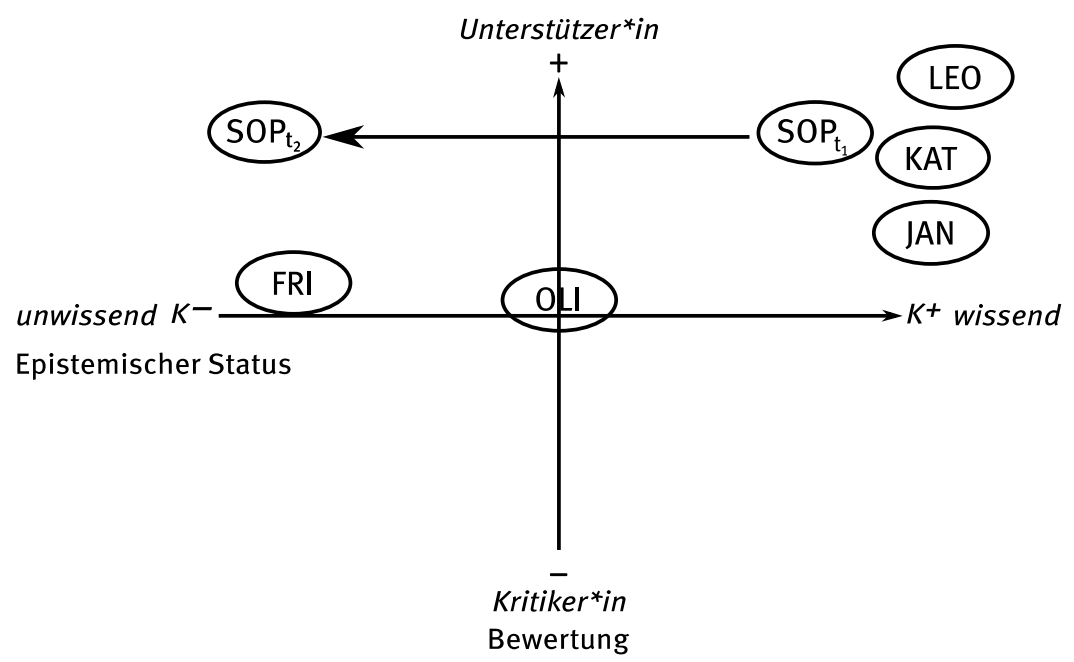

Abb. 7.6: Relativen Bestimmung epistemischer und evaluativer Positionierungen der Interagierenden in Bezug auf die Serie 13 REASONS WHY anhand des entwickelten Koordinatensystems. Die Stärke der Linien um die Sprecher*innensiglen zeigt die Intensität der Positionierungen an. Die Indizes $t_{1}$ und $t_{2}$ hinter Sophies Namen sowie der Pfeil deuten den zeitlichen Verlauf ihrer Positionierung an.

Dieser Fall scheint eine Besonderheit darzustellen, da in den meisten anderen Sequenzen der Daten die Teilnehmenden üblicherweise keine hohe epistemische Autorität aus der bloßen Kenntnis des Trailers einer Serie ableiten oder sie ihnen zeitnah wieder abgesprochen wird. Stattdessen ist es wahrscheinlicher, dass in einem solchen Fall die Praktik des Berufens auf die Bewertungen anderer greifen 
würde. Anders als in anderen Fällen, in denen das Beanspruchen epistemischer Autorität zur Bewertung verhandelt und abgesprochen wird (vgl. Analysen in Abschnitt 7.1.3), wird Sophies nachträgliche Revidierung jedoch interaktiv nicht problematisiert, d.h. sie ist erfolgreich mit ihrer Praktik. Das Beispiel zeigt anschaulich auf, wie Gesprächspartizipation in Bezug auf eine konkrete Serie („mitreden können“) möglich ist, auch ohne die Serie gesehen zu haben und ohne den eigenen geringen Kenntnisstand notwendigerweise sofort kommunizieren zu müssen.

Eine ähnliche Form, den eigenen epistemischen Status hochzustufen und ihn abschließend wieder zurückzunehmen, findet sich in folgendem Beispiel:

(27) hab ich AUCH nur so sporAdisch mal geguckt;

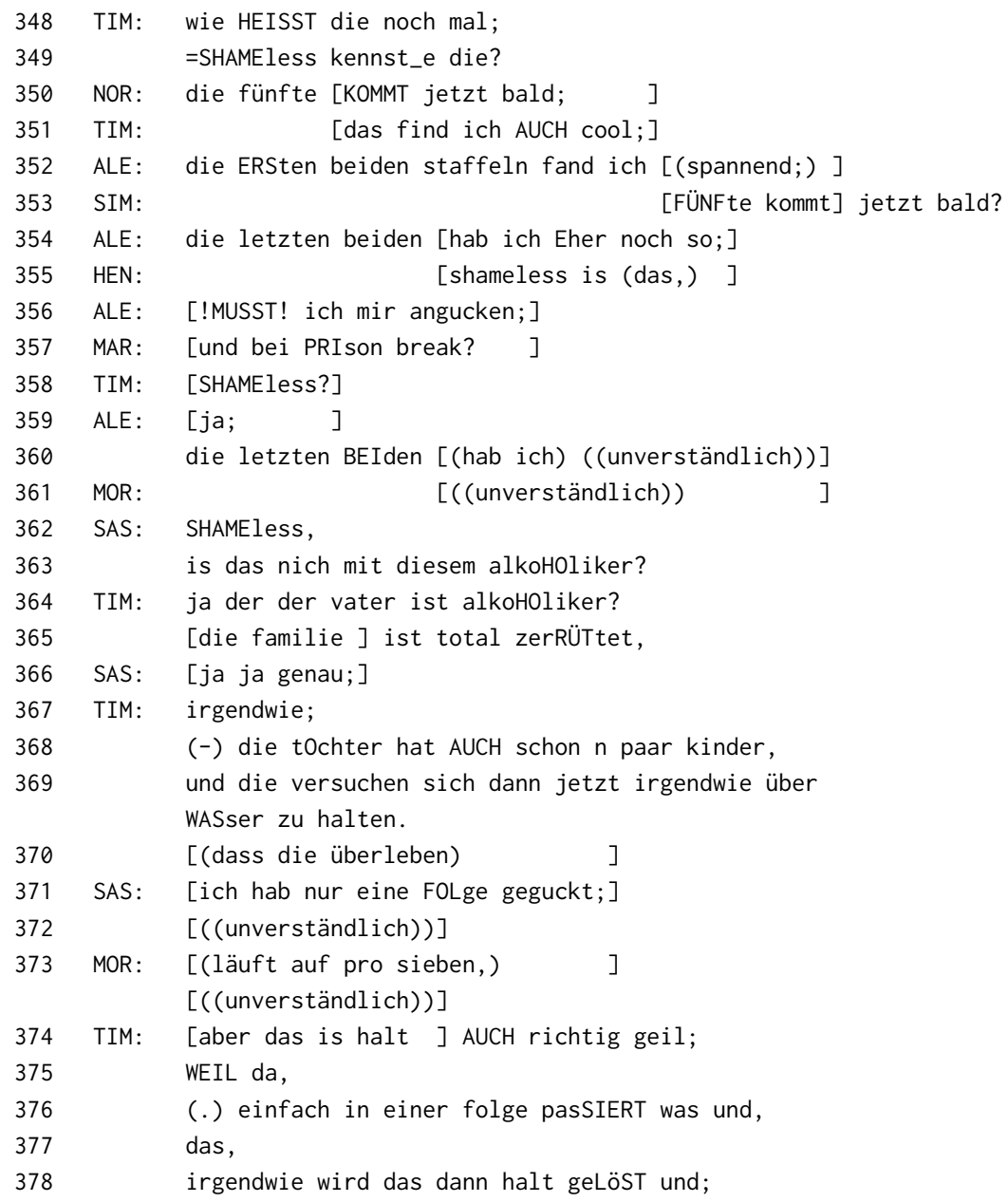


379

380

381

382

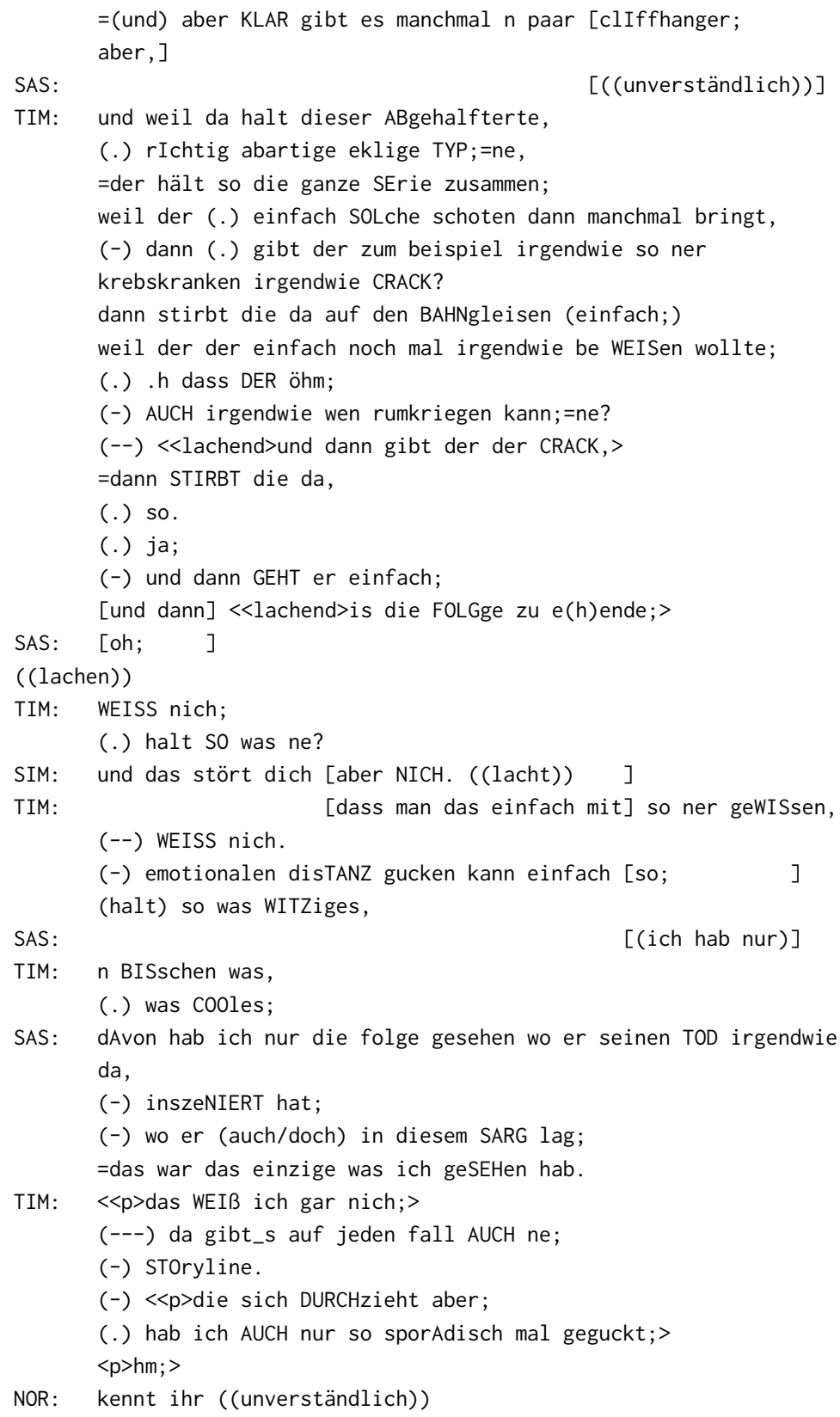


Die Sequenz ist eingebettet in das übergeordnete Gesprächsthema, dass Tim sich rechtfertigt, warum er Serien wie GAME OF THRONES nicht mag. Seine Erklärung ist, dass ihn die Serie nicht packen würde und er insgesamt Cliffhanger n nichts abgewinnen könne (vgl. auch die Analysen in Abschnitt 8.1.3 und Abschnitt 9.1.4). Tim erfragt also hier den epistemischen Status der anderen Teilnehmenden zu der Serie SHAMELESS, die er damit als neues Positionierungsobjekt etabliert, nachdem sich die Diskussion lange Zeit um GAME of THRones drehte, so dass er hier die Praktik Etablieren eines neuen Positionierungsobjekts erfolgreich angewendet hat.

Nachdem die Frage geklärt wurde, ob es um SHAMELESS oder PRISON BREAK geht (Z. 350-361), zeigt Sascha partielles Wissen an, indem er eine Rückfrage zu dem Protagonisten der Serie stellt, auf den er als „diesen alkoHOliker“ (Z. 363) referiert. Tim bestätigt seine Zuordnung und zählt weitere Informationen zur Figurenkonstellation der Serie auf (Z. 367-370, 374-385). Dabei wird sowohl seine evaluative Haltung gegenüber der Serie erkennbar (,aber das is halt AUCH richtig geil; “, Z. 374) als auch seine hochgestufte epistemische Haltung, da er die narrative Struktur der Serie erläutert (Z. 367-380, 382-385) sowie als Beleg für seine Charakterisierung des Protagonisten eine Szene rekonstruiert (Z. 386-396).

Inhaltlich wird dabei deutlich, dass die Serie mit Normverstößen und Tabubrüchen arbeitet, was z.B. an Tims begleitenden Lachpartikeln (Z. 391) sowie an Saschas entsprechender Reaktion „oh“ (Z. 397) oder Simons ironischer Frage „und das stört dich aber NICH; “ (Z. 401) ersichtlich wird. Tim kontert mit der Kategorisierung der Serie als „witzig“ (Z. 405) und damit, dass er sie „einfach mit so ner geWISsen emotionalen disTANZ gucken“ (Z. 402-404) würde (vgl. dazu auch Analyse zu Beispiel 45 in Abschnitt 8.2.4.1). Eine Vergemeinschaftung mit den anderen Gruppenmitgliedern über eine geteilte positive Bewertung der Komik der Serie (wie u.a. in Beispiel 18) bleibt somit aus.

Indem Sascha in einem sequenzerweiternden Turn seinen eigenen epistemischen Status als minimal wissend durch Rekonstruktion einer Folge markiert (Z. 409-412), eröffnet er jedoch Tim die Möglichkeit, weiter über die Serie zu diskutieren und sich möglicherweise über die gemeinsame Bewertung der Episode doch zu vergemeinschaften. Tatsächlich bleibt dieses Angebot aber erfolglos, denn Tim signalisiert mit sehr leiser Stimme, dass er diese Folge offenbar nicht kennt (Z. 413) und äußert eine potentiell sequenzabschließende Bewertung (Z. 414-416). Dann stuft er seinen epistemischen Status zurück, indem er signalisiert, dass er die Serie „AUCH nur so sporadisch mal geguckt“ (Z. 417) hat. Das Zurückstufen wird hier genau wie in Beispiel 26 im weiteren Verlauf interaktiv nicht relevant gesetzt (vgl. Z. 419). Eine mögliche Erklärung für Tims Zurückstufen könnte die dispräferierte Reaktion der Gruppenmitglieder auf seine Schilderungen ,witziger` Szenen der Serie sein. Solche Inkongruenzen zwischen Haltung und Status können in den vorliegenden Daten selten beobachtet werden. Eine ausführliche Analyse einer 
Sequenz, in der sowohl epistemische als auch evaluative Haltungen sukzessive interaktiv herabgestuft werden, findet sich in Abschnitt 7.3.2.

\subsubsection{Zusammenfassung: Positionierungspraktiken zur Beanspruchung von Expertise bei $K^{-}$}

In diesem Abschnitt wurden die vier Praktiken beschrieben:

(1) Anzeigen von partiellem Wissen durch Kommentieren oder Fragen

(2) Berufen auf die Bewertung anderer

(3) Kommunikativ erfolgreiches bzw. erfolgloses Etablieren eines Positionierungsobjekts

(4) Nachträgliches Revidieren des epistemischen Status

Diese Praktiken beziehen sich auf eine ${ }^{\star} n$ Sprecher ${ }^{\star}$ in, die ${ }^{\star}$ der bei implizit oder explizit kommunizierter $\mathrm{K}^{-}$-Position ihren epistemischen Status hochstufen bzw. versuchen, ihn hochzustufen. Dafür können die Interagierenden partielles Wissen in Form von Kommentaren anzeigen oder es mit verschiedenen Graden epistemischer Gewissheit in Form von Fragen an $K^{+}$-Teilnehmende, verpacken'. Anders als es in der Praktik Absprechen von fremder epistemischer Autorität zur Bewertung bei $K$ - der Fall ist, bleiben sie jedoch meistens in der epistemisch hierachieniedrigeren Position. Da sie über mangelndes Wissen zu einer fundierten Bewertung verfügen, können sie die Bewertung als nicht ihr eigenes, subjektives Urteil markieren, sondern sich selbst in Relation zu der Bewertung abwesender Dritter positionieren. Wenn sie weder Wissen noch Bewertungen bezüglich der gerade diskutierten Serie äußern können oder wollen, bleibt ihnen die Praktik des Etablieren eines Positionierungsobjekts, zu dem sie einen höheren epistemischen Status besitzen.

Nicht alle Praktiken sind jedoch immer kommunikativ erfolgreich. Nicht durch eigene Rezeption gewonnenes Wissen und Bewertungen im Sinne eines präferierten epistemischen Zugangs können leichter angefochten und zurückgewiesen werden, der versuchte Themenwechsel kann scheitern. Erfolgreich ist dagegen Sophies Praktik in Beispiel 26. Obwohl sie erst nachträglich anzeigt, die Serie nicht zu kennen, kann sie mühelos partizipieren und sich in eine epistemisch höhere Position bringen, als andere $K^{-}$-Beteiligte. Wird diese Sequenz jedoch mit Beispiel 27 verglichen, so lässt sich resümieren, dass diese Praktiken offenbar eher dann zielführend sind, wenn die Wissenskommunikation auf einer vergleichsweise oberflächlichen (Handlungs- bzw. Plot-)Ebene bleibt und nicht zu konkret in Bezug auf einzelne Episoden oder Szenen wird.

Primäres Ziel dieser Praktiken scheint daher das der Partizipation am aktuellen diskursiven Gegenstand zu sein, dem das punktuelle Etablieren von Gemeinschaft 
(wie bei Beispiel 27) oder Distinktion (wie manche der in Abschnitt 7.2.1 angeführten Beispiele) nachgeordnet ist. Nachdem bisher Praktiken im engeren Kontext für einzelne Teilnehmende, die sich entweder als $K^{+}$oder $K^{-}$positioniert haben, herausgearbeitet wurden, werden nun anhand von Fallbeispielen gruppendynamische Prozesse der Vergemeinschaftung und Distinktion betrachtet.

\subsection{Gruppendynamische Positionierungsprozesse zur Herstellung von Vergemeinschaftung und Distinktion - Drei Fallstudien}

Während sich die Analysen in Abschnitt 7.1 und 7.2 vor allem auf die Praktiken einzelner Sprecher`innen konzentriert haben, liegt in diesem Abschnitt der Fokus auf gruppendynamischen Prozessen, in die diese Praktiken eingebettet sind. Die drei hier präsentierten Fallanalysen bilden dabei Eckpunkte verschiedener Varianten der Etablierung von Gemeinschaft und Distinktion einer Gruppe und stellen nicht Exemplare größerer Kollektionen dar. Mit ihrer Analyse soll in explorativer Weise gezeigt werden, wie sich gruppendynamische Prozesse über epistemische und evaluative Positionierungspraktiken der Teilnehmenden verändern und insbesondere dafür funktionalisiert werden, sich mit der ganzen Gruppe oder einer Subgruppe $\mathrm{zu}$ vergemeinschaften und/oder davon abzugrenzen.

Die Unterkapitel sind wie in Abschnitt 7.1 nach den Serien, die in den Sequenzen verhandelt werden, benannt. In Abschnitt 7.3.1 wird anhand dreier Transkriptausschnitte nachgezeichnet, wie sich die Gruppe über das Teilen von (Kindheits)Erinnerungen an die Serie AvATAR vergemeinschaftet. In der Fallstudie zu SHERLOCK (Abschnitt 7.3.2) wird analysiert, wie die Gruppe sukzessive ihre Bewertung und ihren epistemischen Status herabstuft und aneinander anpasst. Anhand der Fallstudie zu How I MET YOUR MOTHER vs. TÜRKISCH FÜR ANFÄNGER (Abschnitt 7.3.3) kann gezeigt werden, wie sich zwei Subgruppen bezüglich der Bewertung beider Serien im Vergleich bilden, die auf vergnügliche Art und Weise ihren Dissens aufrechterhalten.

\subsubsection{AVATAR - Vergemeinschaftung durch Verweisen auf geteiltes Wissen und geteilte Bewertungen}

Das in diesem Abschnitt analysierte Fallbeispiel ist mehrere Minuten lang. Die komplette Sequenz (MNG11, Z. 1134-1263) kann in Anhang IV nachgelesen werden. Grundsätzlich ist die Sequenz von Überlappungen, Simultansprechen und abgebrochenen Äußerungen durchzogen. Damit stellt sie in Bezug auf die Organisation des 
Gesprächs eine Ausnahme im Korpus dar. Gründe dafür könnten zum einen in den generellen Gruppendynamiken dieser Schülerinnen und Schüler liegen, die sehr konsensbemüht und auf gruppenbezogene Vergemeinschaftung abzielend agieren. Eine andere Begründung mag darin liegen, dass es sich hierbei - und das ist tatsächlich im gesamten Korpus einmalig - um eine Serie handelt, die alle Beteiligten gleichermaßen sehr gut kennen und sehr positiv bewerten. Anders als für die in Abschnitt 7.2 dargestellten Praktiken muss also keine zusätzliche ,Hürde‘ in Form des Hochstufens des epistemischen Status bei $K^{-}$genommen werden, um partizipieren zu können. Alle Beteiligten befinden sich - bildlich gesprochen - in dem oberen rechten Quadrant des in Abschnitt 6.4 dargestellten Koordinatensystems und positionieren sich als wissende Unterstützer^innen. Genau genommen handelt es sich bei dieser Sequenz also um einen Spezialfall der von allen Beteiligten genutzten Praktik Managen divergenter Wissens(be)stände, die hier abgewandelt wird zum Managen gleicher Wissens(be)stände. Die sich daraus ergebende Positionierung als Gruppe soll im Folgenden mikroanalytisch rekonstruiert werden.

Der Abschnitt ist folgendermaßen strukturiert: Zunächst wird die gesamte Sequenz in ihrem Ablauf kurz zusammengefasst. Anschließend wird anhand dreier chronologisch gewählter Ausschnitte nachgezeichnet, wie die Beteiligten sich als Gruppe konsensuell positionieren. Der Analysefokus liegt darauf, wie sie Gemeinschaft und Zugehörigkeit darüber herstellen, dass sie die Serie als bei allen Beteiligten im common ground befindlich behandeln (Beispiel 28). Dafür stellen sie zum einen interaktiv Emotionalität und Gefühle über geteilte Rezeptionserfahrungen her (Beispiel 29) und zum anderen stufen sie ihre Zweitbewertungen nicht nur verbal, sondern überwiegend mittels multimodaler Ressourcen hoch (Beispiel 30).

Die Sequenz beginnt damit, dass Martha den epistemischen Status der Gruppe zu AvATAR erfragt, woraufhin sich alle anderen verbal oder nonverbal als $K^{+}$positionieren und ihre positive Bewertung kommunizieren (Z. 1134-1150). Es folgen Verweise auf einzelne Szenen oder Episoden der Serie (Z. 1151-1190) und auf deren ,Suchtfaktor‘ (Z. 1191-1206). Abschließend diskutieren sie, wie die Gruppenmitglieder die Serie als Kinder rezipiert haben (Z. 1261-1261). Die Sequenz schließt damit, dass Bilel als neues Positionierungsobjekt PowER RANGERS etabliert (Z. 1262-1263).

(28) kennt ihr avaTAR,

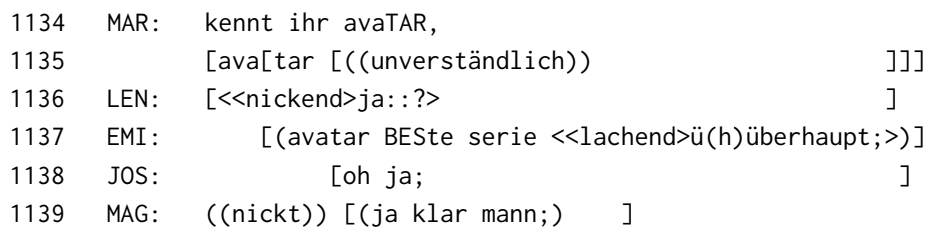




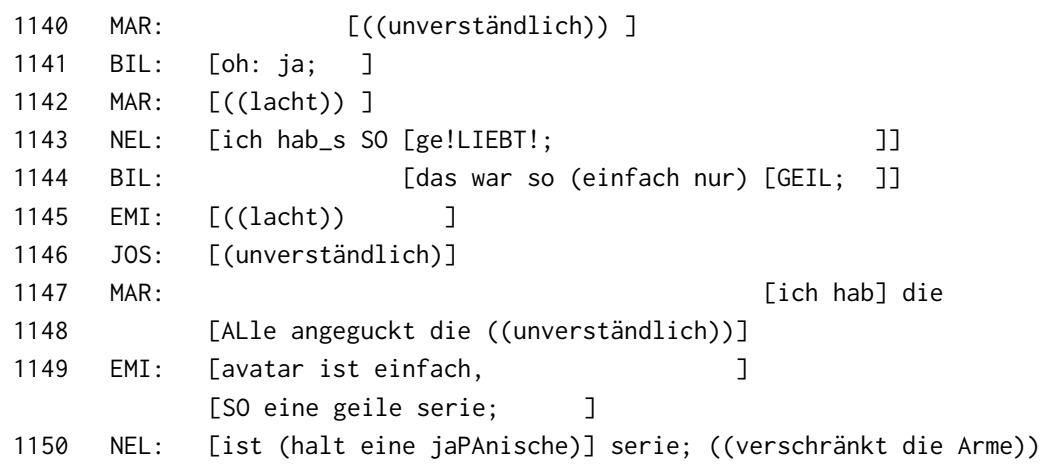

MNG11, 20:20-20:34

Die Teilnehmenden behandeln die Serie als im common ground der Gruppe verankert. So impliziert beispielsweise Magnus' Reaktion auf Marthas Frage mit ,ja klar mann; “(Z. 1139), dass die Kenntnis der Serie als selbstverständlich angenommen werden kann. Auch die darauf folgenden epistemischen Haltungen der von Martha adressierten Teilnehmenden signalisieren mittels einer hohen Gewissheit und Glaubwürdigkeit eine derart fundierte Kenntnis der Serie, dass sie damit zugleich auch eigenständige epistemische Autorität beanspruchen: „ich hab_s so ge!LIEBT!; “ (Z. 1143), „das war so (einfach nur) GEIL; (Z. 1144). Nach Lenis nickendem und mit steigender Intonation artikulierten ,ja::?“ (Z. 1136) steigern sich die Bewertungen mit extreme-case Formulierungen (Z.1137), Intensifiern (Z. 1143, 1149) und sehr starken Betonungen (Z. 1143, 1151). Nelli verwendet die generalisierende Partikel „halt“ für ihre Kategorisierung der Serie als „jaPAnische Serie“, ${ }^{8}$ womit sie serienexternes Wissen (Abschnitt 7.1.2) einbezieht. Im Zusammenspiel mit der Bewegung ihrer Arme, die sie vor dem Körper verschränkt und einem schräg nach oben gewandten Blick zeigt sie damit mehrere Aspekte an: Zum einen impliziert sie, dass ihre positive Bewertung auf den Produktionsort zurückzuführen ist. Zum anderen verweist das „halt“ darauf, dass es sich dabei damit um eine mit den anderen geteilte Einschätzung handelt. Zugleich artikuliert sie die Bewertung ihrer Körperposition und -bewegung folgend - ohne eindeutige Adressierung an ein oder mehrere Gruppenmitglied(er) und damit möglicherweise auch nur für sich selbst bestimmt. Sie beginnt bereits damit, Privatheit in dieser ansonsten als weitgehend öffentlich gerahmten Interaktionssituation herzustellen (vgl. Abschnitt 6.1.2). Weiterhin verweisen die Bewertungen stets auf Wissenselemente, die

8 Tatsächlich handelt es sich jedoch um eine US-amerikanische Serie, die den japanischen Zeichenstil imitiert. 
sich aus der Rezeption der Serie speisen, ohne sie näher zu elaborieren. Solches Wissen besteht beispielsweise aus Rekonstruktionen einzelner Szenen (Z. 1151, 1161, 1179-1187, vgl. auch Beispiel 29) oder als typisch markierter Gesten der Figuren (Z. 1169, 1215-1223, vgl. dazu auch Beispiel 30). Die geäußerten Wissenselemente sind dabei von Abbrüchen gekennzeichnet, in hohem Maße gestisch begleitet und sehr voraussetzungsreich.

Exemplarisch soll nun anhand des Ausschnitts in Beispiel 29 mikroanalytisch herausgearbeitet werden, wie die Beteiligten diese Wissenselemente einerseits als geteiltes Wissen behandeln, andererseits die damit verbundenen Emotionen als in ihrer subjektiven Rezeption begründet und somit in ihrem eigenen (Rezeptions-)Erleben verankert kommunizieren. Die Gesten wurden so präzise wie möglich beschrieben, die senkrechten Striche „|“ markieren die verbalen Abschnitte, während derer sie vorkommen.

(29) wo die MONDprinzessin gestorben ist,

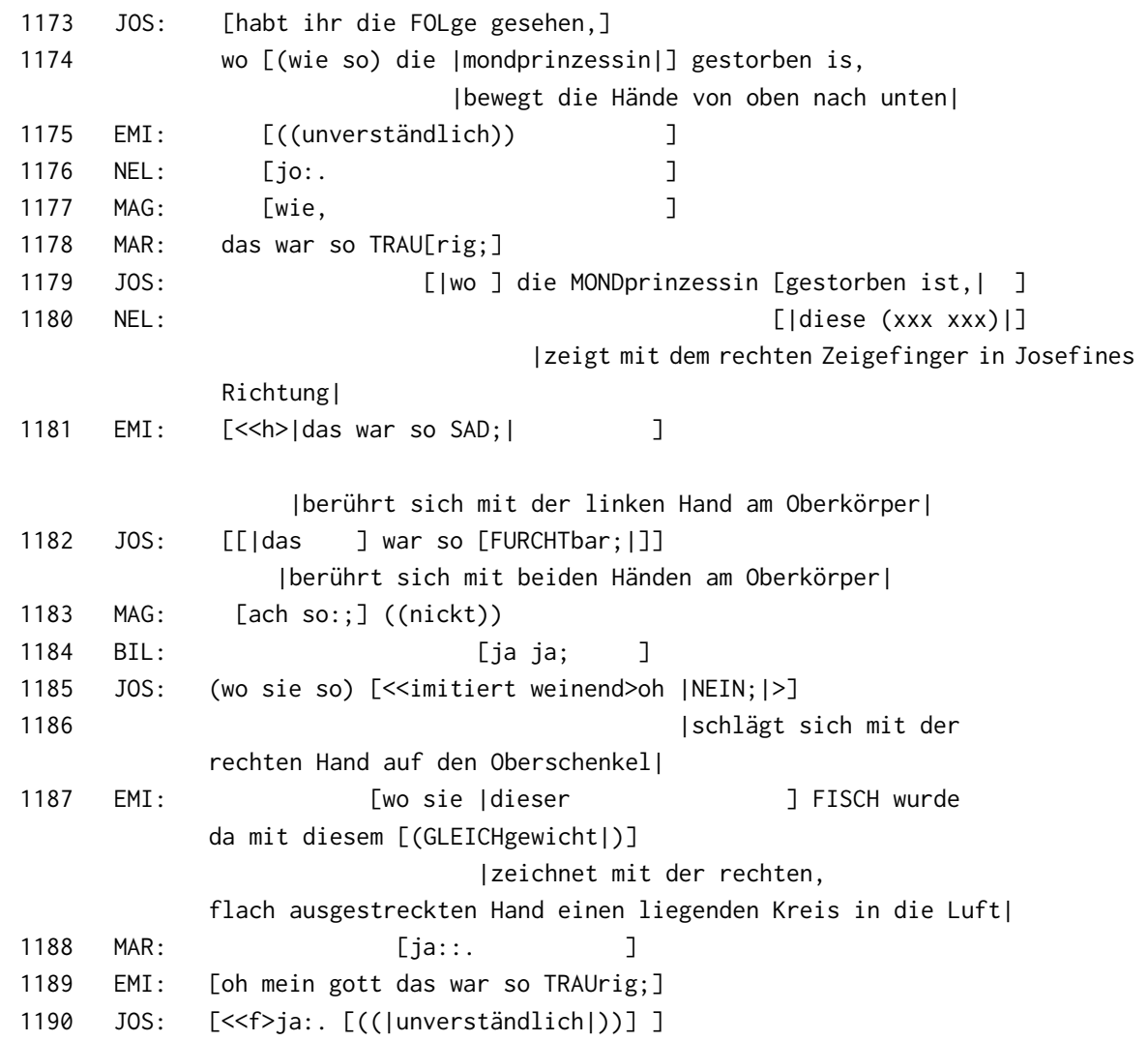


1191

1192

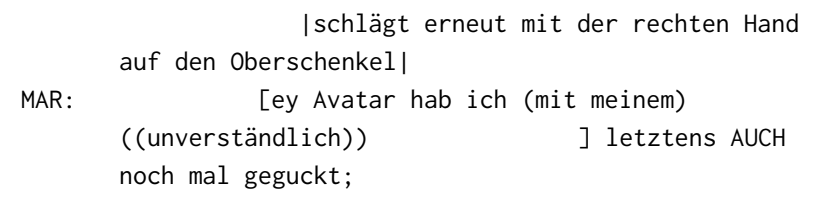

|schlägt erneut mit der rechten Hand

(n)

Josefine erfragt hier den epistemischen Status der anderen in Bezug auf eine Episode der Serie, in der die Figur Yue (die Mondprinzessin) stirbt (Z. 1173-1174). Auf Magnus' akustische Verständnisfrage hin wiederholt sie ihre Äußerung (Z. 1179). Währenddessen vollzieht sie mit beiden Händen folgende Geste: Sie streckt die Arme vor dem Oberkörper leicht angewinkelt aus, die Handflächen zeigen schräg nach innen. Im Takt ihrer Intonation bewegt sie die Hände nach oben und nach unten. Damit erzeugt sie den Eindruck, als eröffne sie die ,Bühne` für die Vorstellung dieser Szene. Während Nelli zwar etwas Unverständliches, aber mit deiktischer Geste eindeutig an Josefine Adressiertes äußert (Z. 1180), reagiert Emilia mit sehr hoher Stimme mit der Bewertung „das war so SAD“; (Z. 1181). Josefines Zweitbewertung zeigt durch Hochstufen Zustimmung: „das war so FURCHTbar“ (Z. 1182). Beide Teilnehmerinnen passen dabei nicht nur ihre Bewertungen, sondern auch ihre Gesten aneinander an, indem ihre Hände jeweils zu ihrem Oberkörper zeigen und sich dort (fast) selbst berühren.

Im Folgenden stellen sie interaktiv geteiltes emotionales Erleben der Szene her, das nicht vieler Worte bedarf, sondern fast ausschließlich auf einer multimodal erzeugten Vergewisserung der gemeinsamen Bewertung beruht. Josefine verweist diffus auf die sterbende Figur (,wo sie so“, Z. 1185) und kommentiert dann ihre Rezeptionssituation, indem sie mittels inszenierender Redewiedergabe ihr früheres Rezeptions-Ich (vgl. dazu auch Abschnitt 8.2.4.1) zitiert und dessen Reaktion auf die Episode schildert. Emilia präzisiert Josefines begonnenen Turn mit ,wo sie dieser FISCH wurde da mit diesem GLEICHgewicht“ (Z. 1187) inhaltlich. Begleitend „malt“ sie einen virtuellen Kreis in die Luft (in Streecks Terminologie entspräche das dem darstellenden Gestentyp „drawing“, vgl. Streeck 2009: 136). Relevante Informationen für die Vergemeinschaftung - die geteilte Trauer über das Schicksal der Figur - werden hier also überwiegend gestisch transportiert. Sowohl Josefine als auch Martha ratifizieren Emilias Beitrag (Z. 1188, 1190) und Emilia wiederholt als Sequenzabschluss ihre Bewertung der Episode, die sie dieses Mal auf Deutsch übersetzt kommuniziert: „oh mein gott das war so TRAUrig; “ (Z. 1189). Auch Bilel und Magnus signalisieren ihre Kenntnis der Folge (Z. 1183, 1184), allerdings partizipieren sie nicht an der evaluativen und Affekt konstruierenden Interaktion zwischen Josefine und Emilia. Auffällig sind sowohl Emilias als auch 
Josefines Selbstberührung ihrer Oberkörper mit den Händen (s.o.). Im Kontext dieser Sequenz können sie so gedeutet werden, dass beide hier ihre subjektiv und im Rahmen einer privaten Rezeptionssituation wahrgenommenen Emotionen öffentlich miteinander teilen.

Hochgestufte Zweitbewertungen sind ein übliches Mittel, um Zustimmung und Zugehörigkeit zu vermitteln (vgl. Abschnitt 4.3). In Beispiel 30 wird gezeigt, wie die sukzessive Hochstufung von Zweitbewertungen nicht nur verbal, sondern auch gestisch und körperlich realisiert werden kann. Der Ausschnitt enthält mehrere Verweise auf als für die Figuren typisch markierte Körper-Bewegungen. ${ }^{9}$ avatar,

(30) alter ich hab (.) früher immer gedacht ich hätte so SUperkräfte wegen

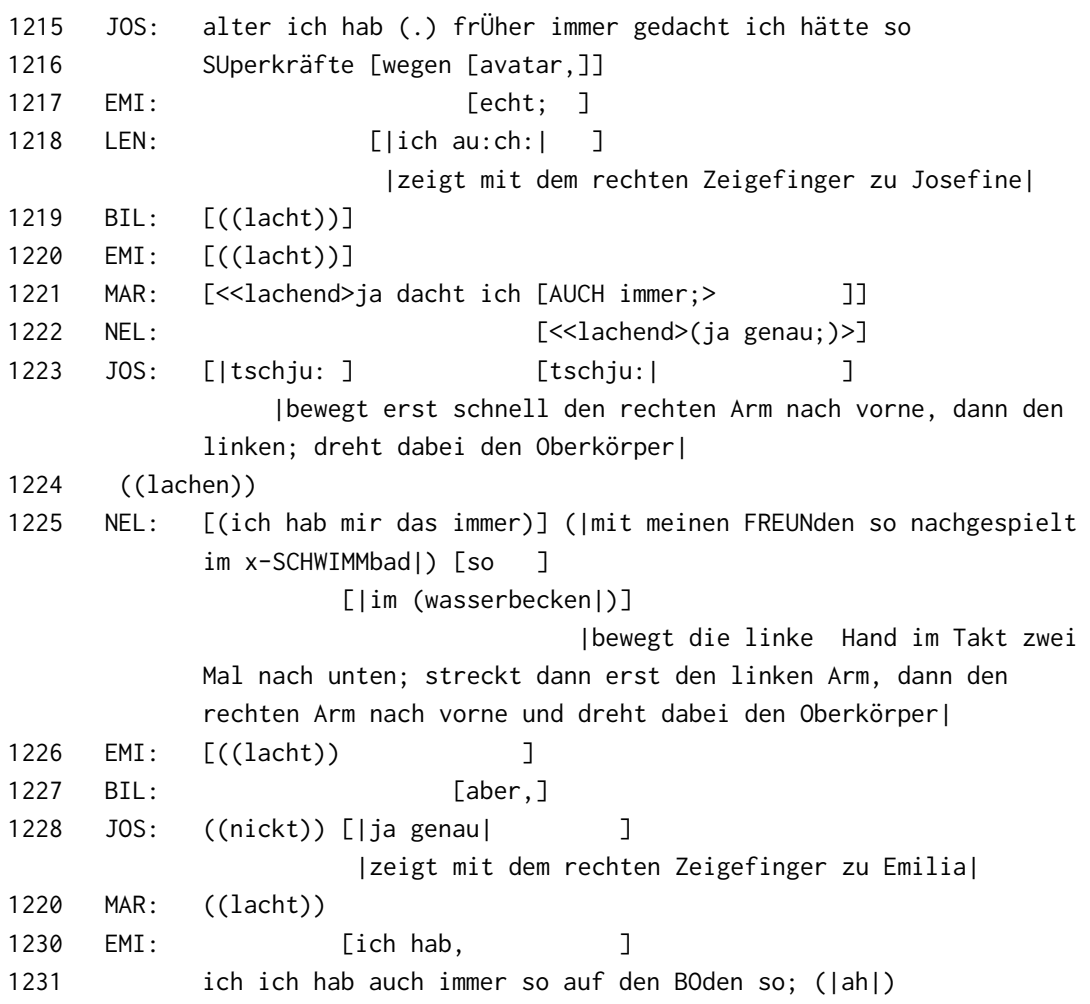

|stampft mit dem

9 Diese typischen Bewegungsmuster können exemplarisch unter folgenden Links eingesehen werden: https://wallpapercave.com/wp/q3CrJJO.jpg, https://wallpapercave.com/wp/wp1905698. jpg [zuletzt abgerufen: 01.06.2021]. 
rechten Fuß auf und streckt ihn dann nach vorne, während sie den Oberkörper nach links dreht|

1232 ((lachen))

1233 BIL: |ich hab das immer Sol gemacht, |zeigt mit dem rechten Zeigefinger zu EMI|

1238 BIL: [((unverständlich))

MAR: $[<<l$ lachend $>$ ich (fand das) immer so (stark mit dem;)]

1241 NEL: [vor allem (wenn du dann immer) so wasser schnell

|HOCH machst, |

| rechte Hand geht schnell von unten nach oben|

dann ((|unverständlich)) (immer so whoo:|)]

|"duckt sich" und klappt beide Hände mit den

Handflächen nach unten zeigend schnell von unten

nach oben|

1243 MAG: [ich ich war so geFLASHT als die angefangen haben mit

Idiesem ( $x x x \quad x x x \quad x x x)$; |

|schnelle Dreh-Bewegung mit dem Zeigefinger|

1245 BIL: ja das war so [GUT; ]

1246 MAG: [alter] ich war [so DRAUßen; ]

1247 MAR: [((unverständlich))]

1248 MAG: ich war die ganze zeit nur HIER so am [| I]

|bewegt beide Hände

einmal schnell vor und zurück;

streckt dann den rechten Arm weit nach schräg oben rechts|

1249 ((lachen))

1250 NEL :

$[((\mid$ lacht $\mid))]$

|klatscht |

1251 MAR: ((lacht)) [(das war |so gut; |)] 
1252 EMI :

[das WEISS ich

] auch noch dieses |.h |

|streckt

erst den rechten Arm nach rechts oben, dann den linken Arm nach links oben; dreht dabei den Kopf|

MNG11, 21:24-22:00

Josefine leitet die Sequenz ein, indem sie sich erneut selbst in Relation zu ihrem früheren Rezeptions-Ich positioniert, das inspiriert durch die Serie von eigenen Superkräften ausging (Z. 1215, 1223), wobei sie strukturell an Leni anschließt, die darlegt, dass sie früher dachte, die Geschichte sei real (Z. 1211). Sie unterstreicht ihre Aussage, indem sie ihren Körper nach vorne lehnt und die Arme abwechselnd nach vorne streckt. Die Bewegung unterstreicht sie zwei Mal begleitend mit dem onomatopoetischen „tschju:“ (Z. 1223) (vgl. Abbildungen 7.7 und 7.8).

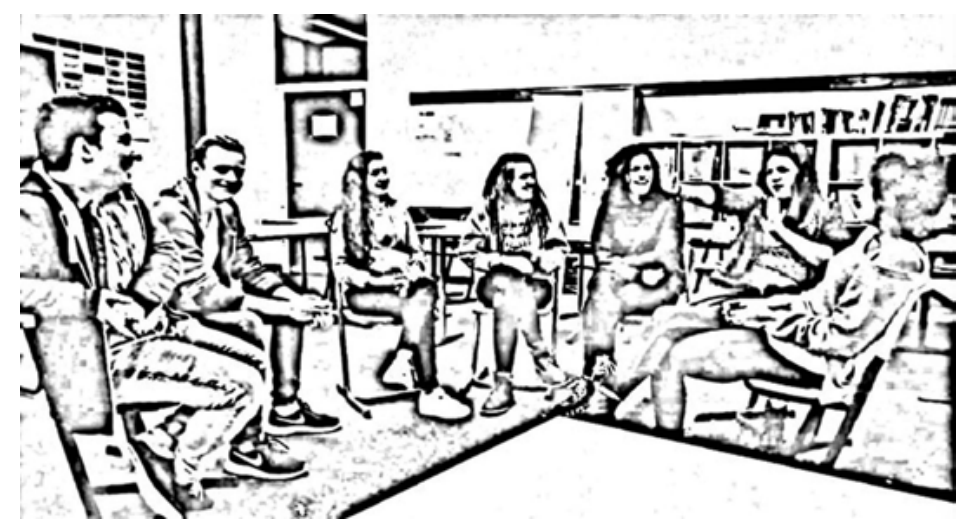

Abb. 7.7: JOS: tschju: ((rechter Arm geht nach vorne)) 


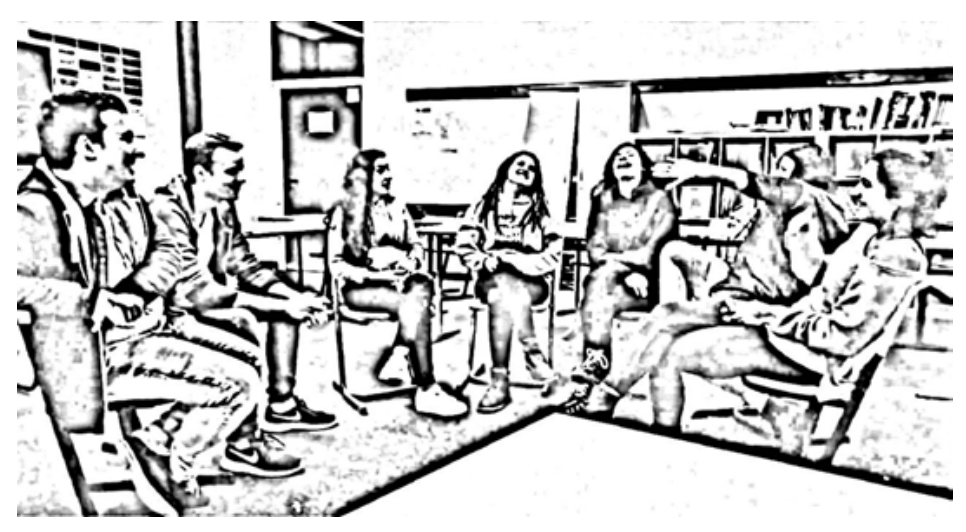

Abb. 7.8: JOS: tschju: ((linker Arm geht nach vorne))

Damit deutet sie eine in der Serie von Figuren typischerweise durchgeführte Bewegung an, wenn sie ihre Superkraft anwenden. Nelli greift im Folgenden Josefines Gestik auf und bettet sie in ein als iterativ - „immer“ - gerahmtes Szenario ein, in dem sie in ihrer Kindheit mit ihren Freunden im Schwimmbad ${ }^{10}$ Szenen aus der Serie nachgespielt hat (Z. 1225; vgl. Abbildung 7.9).

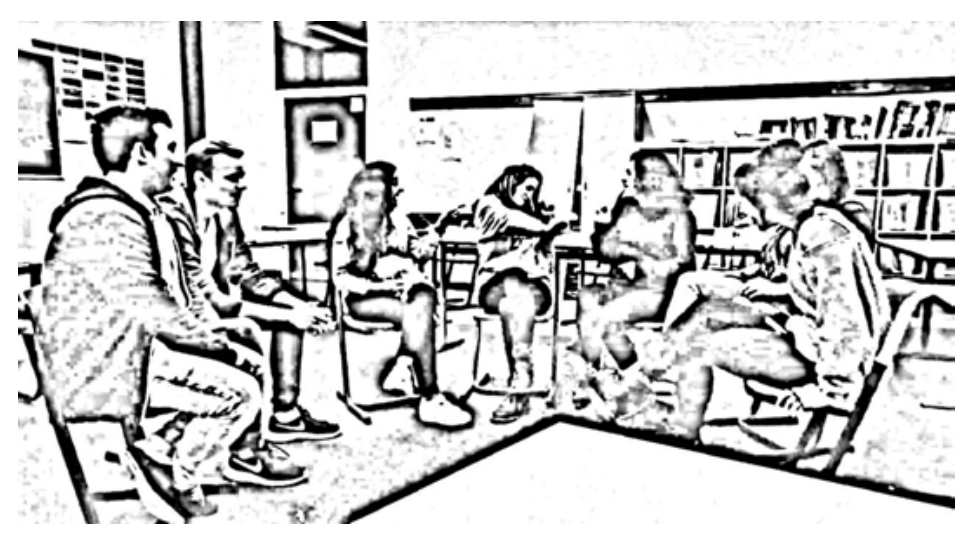

Abb. 7.9: NEL: ich hab mir das immer mit meinen FREUNden so nachgespielt

10 Anmerkung: Hier nennt Nelli den Namen eines lokalen Schwimmbads, der im Transkript aus Datenschutzgründen entfernt wurde. 
Im weiteren Verlauf der Sequenz ,bewegt' sich nun die Imitation dieser FigurenGesten durch die gesamte Gruppe hindurch, wobei jede ${ }^{\star}$ Teilnehmer ${ }^{\star}$ in sie leicht modifiziert. Emilia imitiert ein Stampfen auf den Boden (Z. 1231), was Martha aufgreift und verstärkt (Z. 1239), während Bilel an Nelli anknüpft und ebenfalls inszeniert, wie er als Kind mit Wasser Szenen aus der Serie nachgespielt hat (Z.12331236). Magnus bezieht seine Geste wiederum auf die Ausgangsbewegung von Josefine und kommentiert sie ebenfalls auf seine frühere Rezeptionssituation bezogen positiv (Z. 1246, 1248; Abbildung 7.10).

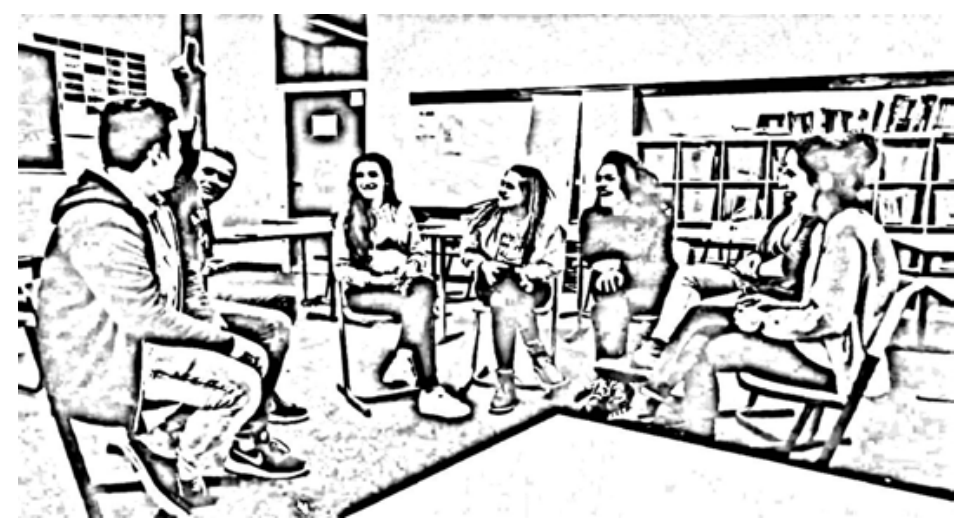

Abb. 7.10: MAX: ich war die ganze zeit nur HIER so am,

Diese Gesten, die in Anlehnung an Streeck (2009: 144) als „acting“ bezeichnet werden können, werden im Laufe der Sequenz immer großzügiger und ausladender. Damit verhalten sie sich analog zu heraufstufenden Zweitbewertungen und Bestätigungen auf verbaler Ebene. Die Gesten übernehmen durch ihr Alignment die Funktion, sich geteilter Bewertung und geteilten common ground s zu vergewissern, der als Auslöser für Kindheitserinnerungen dient.

Insgesamt wird die gruppendynamische Vergemeinschaftung hier durch drei wesentliche Punkte bedingt und durchgeführt: Erstens wird die Serie als bei allen als in gleichem Maße im common ground verankert behandelt, es findet kein expliziter Ausgleich von Wissensasymmetrie statt und niemand stellt den epistemischen Status einer Person in Frage. Zweitens stellen sie interaktiv Emotionalität und Gefühle über ihre geteilten Rezeptionserfahrungen her. Damit rückt das interaktive Ziel der vergnüglichen Vergemeinschaftung über ,joint remembering“ (vgl. Edwards und Middleton 1986, Bietti 2011) bzw. über mehrstimmige Rekonstruktionen (nach Keppler 1994; vgl. Abschnitt 2.2.2 und 2.2.3) in den Vordergrund. Das Rezeptionserleben einer Serie ist eigentlich rein in der epistemischen Domäne der 
Teilnehmenden angesiedelt (vgl. Abschnitt 4.3) und damit mittelbar nach außen nicht zugänglich. Es wird in dieser Sequenz aber über das wechselseitige Bestätigen geteilter Emotionen intersubjektiv erfahrbar gemacht (vgl. ausführlichere Überlegungen dazu in Abschnitt 10.3). Drittens stufen sie ihre Zweitbewertungen mittels multimodaler Ressourcen hoch. Das immer ausladender werdende, vergnügliche Zitieren der Figuren-Geste ist dabei ausschlaggebend für die Vergemeinschaftung als Gruppe im Sinne von .

\subsubsection{SHERLOCK - Vergemeinschaftung durch interaktives Anpassen von Wissen und Bewertungen}

In diesem Abschnitt wird ein Fallbeispiel analysiert, in dem zu beobachten ist, wie Mitglieder der Gruppe interaktiv sukzessive ihre Bewertung der Serie SHERLOCK anpassen und interaktiv herunterstufen. Indem die Teilnehmenden ihre Bewertung verschieben, gestalten sie aus einer potentiellen Distinktionsmöglichkeit (unterschiedliche Bewertungen als Ausgangslage) eine Vergemeinschaftungsaktivität.

Die Analyse ist folgendermaßen strukturiert: Zunächst wird die Sequenz in Beispiel 31 kurz zusammengefasst. Der Analysefokus liegt darauf, wie Sevcan und Verena Gemeinschaft und Zugehörigkeit (vor allem) mit Ole darüber herstellen, dass sie sowohl ihre evaluative als auch ihre epistemische Haltung jeweils sukzessiv an die vorherigen Äußerungen anpassen. Der sich parallel entwickelnde Gesprächsstrang zum Thema Spoilern wird in Abschnitt 8.2.3 diskutiert. Das Besondere dieses Fallbeispiels im Unterschied zu den meisten anderen Daten im Korpus (Abschnitt 7.1 und 7.2) liegt darin, dass dem Anzeigen ausreichenden epistemischen Zugangs zu der Serie eine untergeordnete Rolle zur Bewertung zukommt und stattdessen die evaluative Übereinkunft zur Vergemeinschaftung im Vordergrund steht.

(31) SHERlock is geil;

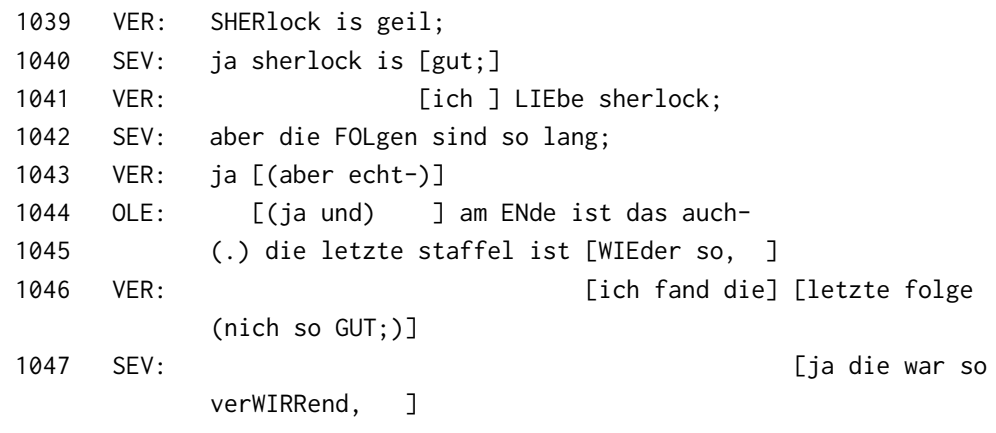




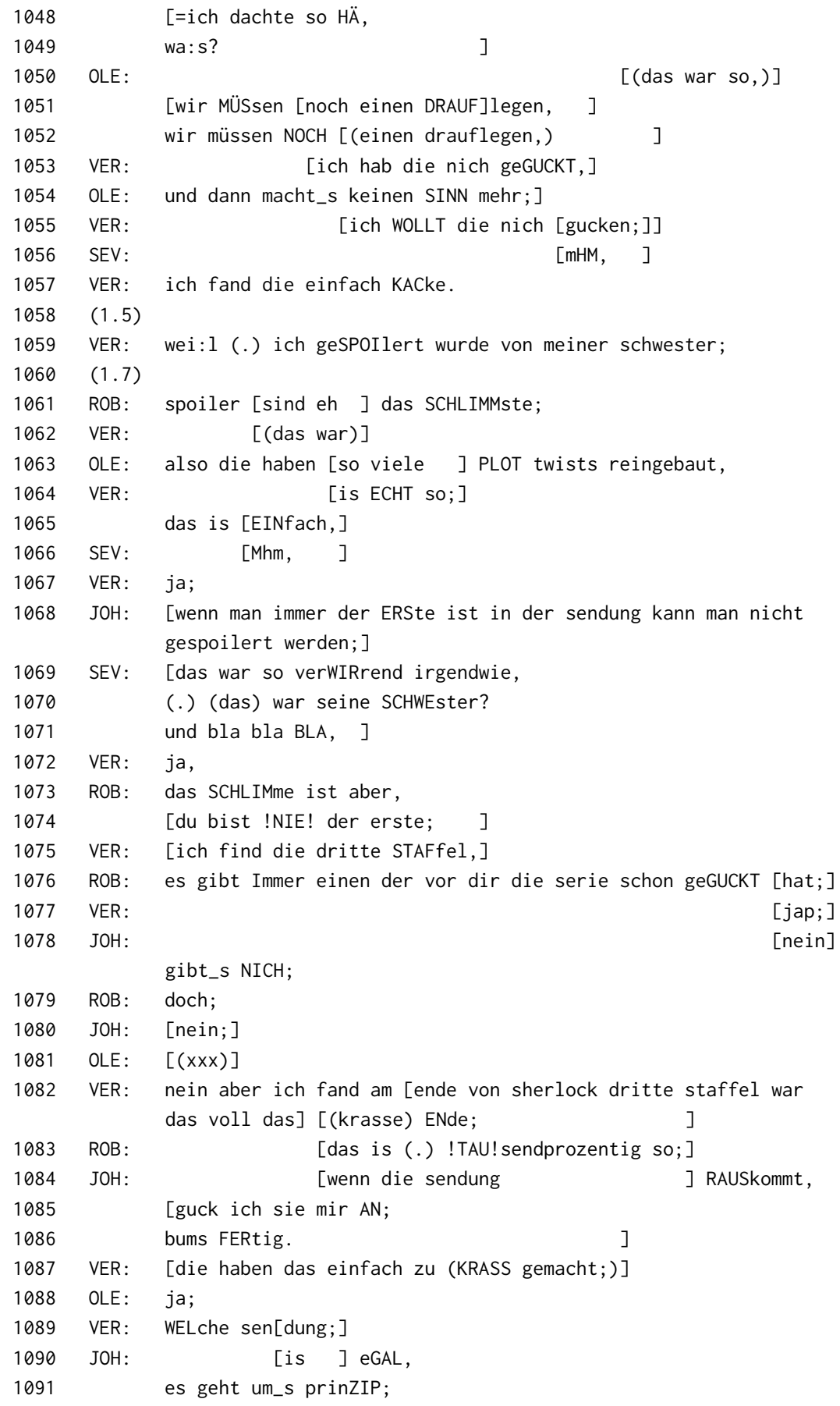


Die Sequenz beginnt damit, dass Verena ihre evaluative Haltung in Bezug auf die Serie SHERLOCK als Positionierungsobjekt markiert und sie gemeinsam mit Sevcan positiv bewertet (Z. 1039-1041). Es schließt sich eine Aushandlungssequenz an, in der Ole, Verena und Sevcan die letzte Episode der aktuellen Staffel im Kontrast dazu negativ beurteilen (Z. 1042-1987). Parallel dazu entwickelt sich ein Gespräch über „Spoiler“ (Z. 1059-1091). Im Folgenden wird zunächst der zeitliche Verlauf von Verenas Äußerungen, dann von Sevcans Äußerungen, in denen sich Veränderungen hinsichtlich ihres epistemischen und evaluativen Status ergeben, analysiert.

Mit der Erstbewertung „SHERlock is geil;“ (Z. 1039) und der als extreme case formulierten Bekräftigung „ich LIEbe sherlock; “ (Z. 1041) signalisiert Verena eine sehr hohe Affinität zu der Serie. Ihre an Oles evaluativer Haltung („die letzte staffel ist WIEder so,“, Z. 1045) angeschlossene bewertende Äußerung (Z. 1046) lässt zudem den Schluss zu, dass sie die letzte Episode rezipiert hat und sie offenbar - im Unterschied zur Gesamteinschätzung der Serie - weniger positiv bewertet. Die darauffolgenden mit anderen Sprechenden überlappenden Turns signalisieren nun eine nachträgliche Revision des epistemischen Status (vgl. Abschnitt 7.2.4) sowie eine im Laufe der Sequenz herabstufende Bewertung der Serie: Verenas epistemische und evaluative Haltung verändern sich von „ich fand die letzte folge nich so GUT (Z. 1046) über „ich hab die nich geGUCKT,“ (Z. 1053) hin zu „ich WOLLT die nich gucken; “ (Z. 1055) und „ich fand die einfach KACke.“ (Z. 1057). Die Begründung für diese Veränderung stellt Verena an das Ende ihrer Redebeiträge. Mit einem langgezogenen „wei:l“ beginnt sie die Erklärung für die epistemische Quelle ihrer Kenntnis der letzten Folge und die darauf basierende Bewertung (vgl. Berufen auf die Bewertung anderer, Abschnitt 7.2.2) sowie die Entscheidung, diese Episode nicht selbst zu rezipieren: Sie wurde von ihrer Schwester „geSPOIlert“ (Z. 1059; Dieser Aspekt ist Gegenstand der Analyse in Beispiel 41 in Abschnitt 8.2.3). Im Folgenden stimmt sie den evaluativen Aussagen der anderen die letzte Staffel betreffend zu (Z. 1064, 1067, 1072) und bekräftigt ihre auf der Kenntnis ihrer Schwester basierende Bewertung des Endes der dritten Staffel (Z. 1075, 1082). Indem sie sich auf ihre Schwester für die Bewertung und als Begründung für das Herabstufen ihres epistemischen Status beruft, verleiht sie ihrer epistemischen Haltung zur Evaluation mehr Glaubwürdigkeit.

Nicht nur Verena, auch Sevcan stuft im Laufe der Sequenz ihre evaluative Haltung herunter (von Z. 1040 zu: 1042, 1069-1071). Auslöser dafür scheint Oles an Sevcans Bemängelung der Länge der Episoden angeschlossene negative Bewertung des Staffelabschlusses zu sein. Er kritisiert die Erzählweise der Serie, die offenbar versucht, sich selbst zu übertreffen und in diesem Zuge die eigene Handlungs- 
logik verlässt (Z. 1044-1045, 1050-1054). ${ }^{11}$ Ole formuliert dazu mittels iterativer Redewiedergabe aus Produzierendensicht: „wir müssen noch einen DRAUFlegen, wir müssen NOCH einen drauflegen" (Z. 1051-1052). Dem stimmen die anderen Teilnehmenden zu und richten sich an dieser Bewertung aus. So stilisiert Sevcan beispielsweise ihre eigene Rezipientinnensicht mittels Redewiedergabe: ,ja die war so verwirrend ich dachte so HÄ, wa:s?“ (Z. 1047-1049) und gibt ein Beispiel (Z. 1069-1071) für ihr Unverständnis der von Ole kritisierten „plot twists“ (Z. 1063) (vgl. dazu auch Abschnitt 8.2.1). Auf diese Weise einigen sich Ole, Sevcan und Verena schließlich darauf, dass die letzte Staffel von SHERLOck als negativ zu werten ist, trotz der zunächst positiven Bewertung, die Sevcan und Verena verbal geäußert haben (Z. 1039-1041), während Ole SHERLOCK als eine Lieblingsserie auf dem zuvor ausgeteilten Fragebogen angegeben hat. ${ }^{12}$

So nutzen die Teilnehmenden verschiedene Wissenselemente und Wissensquellen, um von einer zu Beginn gemeinschaftlich positiven Bewertung über Oles negative Beurteilung eines Aspekts sukzessive Alignment zu signalisieren und ihre eigene evaluative Haltung sowie ihren epistemischen Status - Verena in Bezug auf die tatsächliche Rezeption; Sevcan markiert mehrfach, dass sie der Handlung der letzten Episode nicht folgen konnte - herabstufen. Im Unterschied zu den meisten anderen Sequenzen im Rahmen der Daten spielt der epistemische Zugang hier für die Vergemeinschaftung gegenüber der geteilten und aneinander angepassten Bewertung eine eher untergeordnete Rolle.

Insgesamt erscheinen für das Etablieren von Konsens und damit Anpassen der jeweiligen Positionierungen an die Gruppe die folgenden zwei Aspekte zentral: Zum einen werden die Bewertungen an die anderer Personen mit $\mathrm{K}^{+}$-Status und mit hoher epistemischer Autorität angepasst: Ole positioniert sich durch seine Meta-Perspektivierung im Hinblick auf Produktionspraktiken als Experte (er zieht serienexternes Wissen hinzu, Abschnitt 7.1.2) und die Teilnehmerinnen alignieren ihre Bewertung mit seiner Einschätzung. Zum anderen scheinen hier veränderte Bedingungen von Zeitlichkeit salient zu werden: Verena wechselt im Laufe der Sequenz Tempus und Modus (,ich fand die Folge X“ wird zu „ich hab die Folge nicht geguckt“ wird zu ,ich wollte die Folge nich gucken“). Möglicherweise scheint hier die durch Serialität aufgelöste Linearität der Rezeption (vgl. Abschnitt 2.1.2) in die (verbalsprachliche) Interaktion hineinzuwirken.

11 Schultz-Pernice (2016: 35) bezeichnet diese Strategie als „Eskalation“ in Anlehnung an JahnSudmanns und Kelleters Terminologie der „Dynamik der seriellen Überbietung“ (Jahn-Sudmann und Kelleter 2012), vgl. Abschnitt 2.1.1.

12 Vgl.zur Rolle der Fragebögen bei der Datenerhebung Abschnitt 5.2. Die Fragebögen konnten mittels Pseudonymen den Teilnehmenden zugeordnet werden. 


\subsubsection{HOW I MET YOUR MOTHER VS. TÜRKISCH FÜR ANFÄNGER - Vergemeinschaftung und Distinktion durch Verschieben der Bewertungsebenen}

Das in diesem Abschnitt analysierte Fallbeispiel ist wie in Abschnitt 7.3.1 mehrere Minuten lang; auch hier kann die komplette Sequenz (Z. 478-710) in Anhang VIII nachgelesen werden. Der Fokus der Analyse liegt darauf, wie sich entlang der Serien How I MET YOUR MOTHER und TÜRKISCH FÜR ANFÄNGER zwei Subgruppen (Sophie, Katharina, Friederike und Oliver vs. Leon und Jan) bilden, die sich über ihre jeweiligen Bewertungen voneinander abgrenzen und diese Distinktion über einen längeren Zeitraum aufrechterhalten. Die Analyse ist folgendermaßen aufgebaut: Zunächst wird die gesamte Sequenz in ihrer Struktur kurz zusammengefasst. Anschließend wird aufgezeigt, wie sich die zwei Gruppen interaktiv formieren (Beispiel 32) sowie oppositionell positionieren (Beispiel 33) und mit welchen Ressourcen sie die Positionierungen über das Aushandeln von Bewertungen und Argumentationen aufrechterhalten (Beispiel 34).

Die Sequenz beginnt damit, dass Leon auf die Frage, ob die Gruppenmitglieder Serien in englischer oder deutscher Sprache rezipieren, How I MET YOUR MOTHER als Beispiel für eine Serie, die er auf Deutsch anschaut, nennt (Z. 478-490). Sophie, Friederike, Katharina und Oliver kommunizieren ihre negative Haltung der Serie gegenüber, woraufhin Leon seine positive Einschätzung verteidigt. Es entfaltet sich eine argumentative Sequenz, bei denen die Unterstützer und die Kritiker*innen auf je andere Aspekte Bezug nehmen (Z. 491-645). Leon kritisiert schließlich die Qualität der von Katharina und Sophie als Favorit angegebene Serie TÜRKISCH FÜR ANFÄNGER als gleichermaßen niedrig (Z. 646-655), woraufhin sich ein interserieller Vergleich zwischen den beiden Produktionen entwickelt, die von den beiden Subgruppen gegensätzlich bewertet werden (Z. 656-709). Die Sequenz wird beendet, indem Jan FAMILY GuY als neues Positionierungsobjekt etabliert (Z. 710). Im Folgenden wird zunächst der Beginn der Sequenz analysiert, um zu zeigen, wie sich die beiden Subgruppen bilden.

(32) sowas find ich RICHtig schlimm;

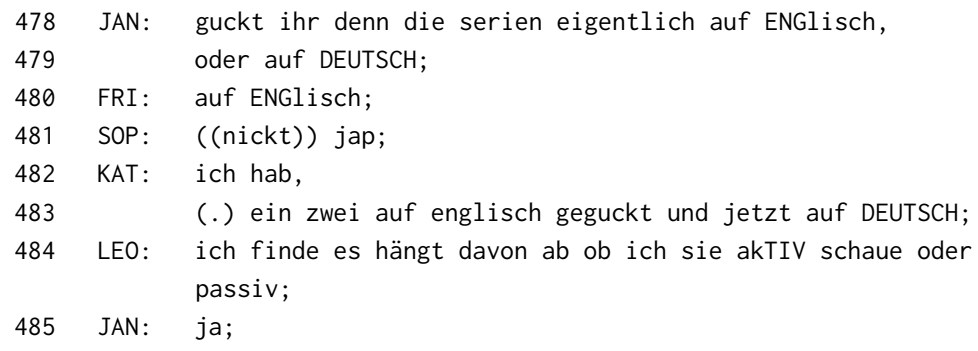




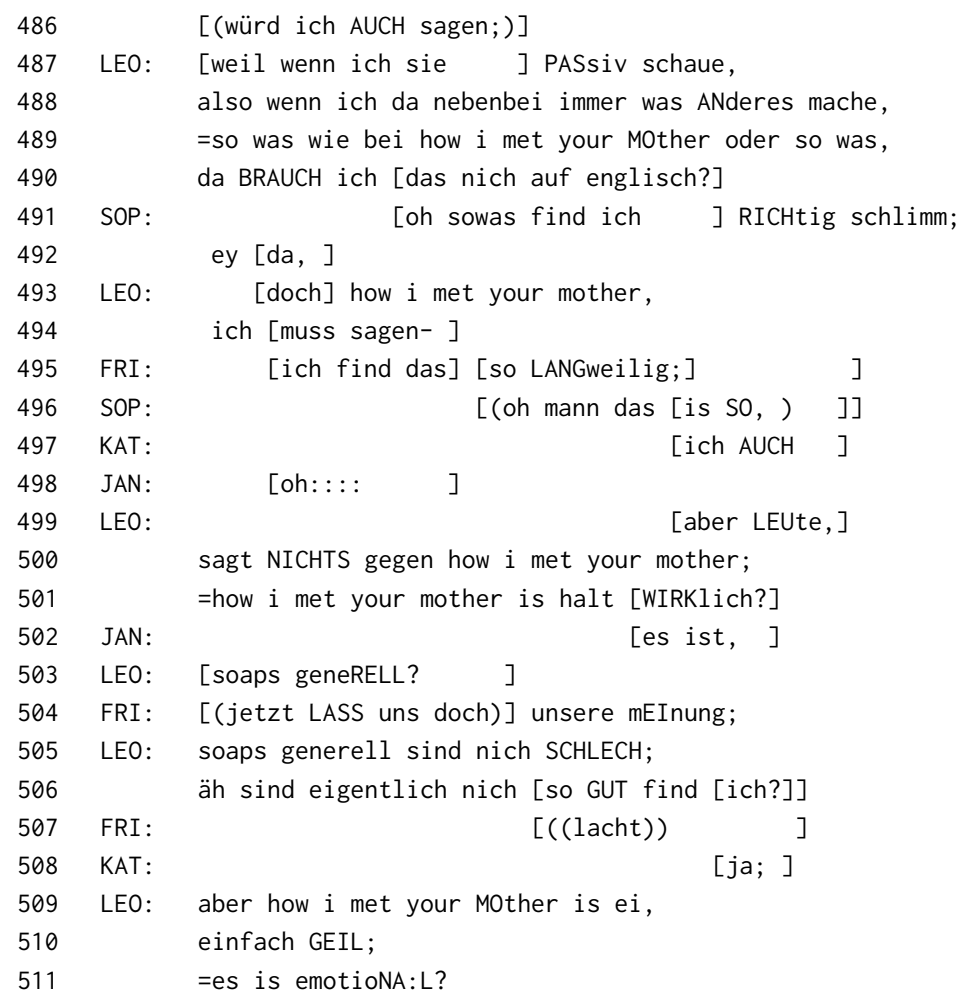

Interaktiver Ausgangspunkt für die Bildung der Subgruppen ist Leons Äußerung, er würde How I MET YOUR MOTHER gerne nebenbei rezipieren (Z. 487-490). Sein Fokus liegt damit auf der Beantwortung von Jans Frage nach der bevorzugten Sprache: Er etabliert einen „kommt drauf an“-Kontrast, den er als aktiven bzw. passiven Rezeptionsmodus labelt, wobei er How I MET YOUR MOTHER als passiv kategorisiert (Z. 478-490). Mit etwas Verzögerung und überlappend etabliert Sophie dann anschließend an das Ende des Turns, mit dem Leon auf How I MET YOUR MOTHER verweist, einen sehr expliziten, evaluativen Kontrast: „oh sowas find ich RICHtig schlimm;“ (Z. 491). Damit löst sie eine Bewertungskaskade aus (Z. 495-504), in deren Rahmen sich Friederike und Katharina mit ihr vergemeinschaften (Z. 495, 497) - Olivers evaluative Haltung wird erst später deutlich (Z. 548) - und Jan mit Leon eine Allianz eingeht (Z. 498). Die folgenden Positionierungen signalisieren meistens indirekt Zustimmung entweder zu der einen oder zu der anderen Gruppe, die sich entweder als Kritiker*innen oder als Unterstützer^innen positionieren (vgl. Abschnitt 6.4). Explizit wird diese Aufteilung in den Meta-Kommentaren ,jetzt 
LASS uns doch unsere mEInung“ (Z. 504) oder auch „die haben einfach KEInen geschmack;“ (Anhang IV: MNG11, Z. 641) ersichtlich. Die dichotome Zuordnung der Interaktionsbeteiligten zu einer der Gruppen wird hier inkludierend mit dem Personalpronomen uns oder exkludierend mit dem Demonstrativpronomen die vorgenommen.

Zugleich etabliert Sophie mit der indexikalisch vage modalisierten Formulierung „sowas“ (Z. 491) eine Kategorie, die sich im Laufe der Interaktion zu der eindeutigeren Genre-Bezeichnung „soaps“ (Z. 503, später: 509, 559, 640)13 und damit zum Dreh- und Angelpunkt der beiden Fronten entwickelt. Zwar sind sich alle Beteiligten einig, dass die Serie diesem Genre zuzuordnen ist, beide Gruppen gestalten diese Zugehörigkeit jedoch unterschiedlich aus. Während die Kritiker^innen die Serie als Prototyp einer Soap konzeptualisieren und ihr davon ausgehend eine Menge negativer Attribute zuordnen, arbeiten Leon und Jan als Unterstützer daran, sie als eine Ausnahme der Regel, also positiv als nicht so eine Soap zu konstruieren. Letzteres wird an Leons reparierender Turn-Konstruktion „soaps generell sind nich SCHLECH, äh sind eigentlich nich so GUT find ich? aber how i met your mother is ei, einfach GEIL;“ (Z. 505-509) ersichtlich.

Als Eigenschaften nennen Kritiker*innen der Serie insbesondere, dass sie sie „schlimm (Z. 491, 560), „LANGweilig“ (Z. 595, 524, 561, 624) und „voll SCHLECHT“ (Z. 548) finden. Sie bezeichnen die Figuren als „UNsympathisch“ (Z. 521) und „NERvig“ (Z.526), kritisieren die Produktion als „richtig BILlig und, amerikanisches fernsehen für DUMme“ (Z. 571-572, indirekt auch 555, 557) und bewerten den Humor negativ: „schlechte WITze“ (Z. 576, 579, 582). Damit argumentieren sie einerseits auf einer eher subjektiven Bewertungsebene („langweilig“), andererseits weisen sie auch ,objektiv beurteilbare` Qualitätsmerkmale der Serie vor dem Hintergrund produktionsbedingter Eigenheiten von Soaps (,im Hintergrund eingespielte Lacher des Publikums“) zurück (vgl. auch Abschnitt 8.1.5). Die Unterstützer schreiben der Serie dagegen mehrere positive Attribute in Listenintonation zu (Z. 565-569) und widersprechen der Beurteilung des Humors, indem sie ihn gegenteilig als „richtig witzig“ (Z. 554) bewerten. Hauptsächlich argumentiert insbesondere Leon jedoch überwiegend über die emotionale Wirkung der Serie auf ihn als Rezipienten, die er mehrfach hervorhebt (Z. 511-514, 539, 565; vgl. auch Abschnitt 8.2.4.1). Beide Gruppen arbeiten fast durchgängig mit Intensifiern und der Ressource Kontrastieren. Jan und Leon bedienen sich im Rahmen einer Nebensequenz auch der Ressource Rekonstruieren und verweisen mit der Praktik Managen divergenter

13 Vgl. Abschnitt 8.2.2 zur Diskussion der normativen Implikationen, die damit und mit Leons Kategorisierung „kultiVIERte serien“ (Z. 591, vgl. Beispiel 37) in dieser Sequenz einhergehen. 
Wissens(be)stände (vgl. Abschnitt 7.1.1) auf den geteilten common ground in Bezug auf konkrete Szenen der Serie (Z. 509-520).

Insgesamt ist auffällig, dass die Gruppen jeweils unterschiedliche Bewertungsmaßstäbe anlegen. Es wird allerdings erkennbar, dass Leon mehrmals versucht, den Kritiker*innen entgegenzukommen, indem er ihnen zwar bezüglich ihrer Einschätzung von Soaps als generelles Genre zustimmt (Z. 506, 550, 559, 563-564, 580), andererseits aber kontinuierlich weiter dafür wirbt, dass How I MET YOUR MOTHER als Ausnahme anerkannt und interaktiv bestätigt wird (Z. 509, 530-539, 625, 627):

(33) hat einfach $n$ anderen ANspruch;

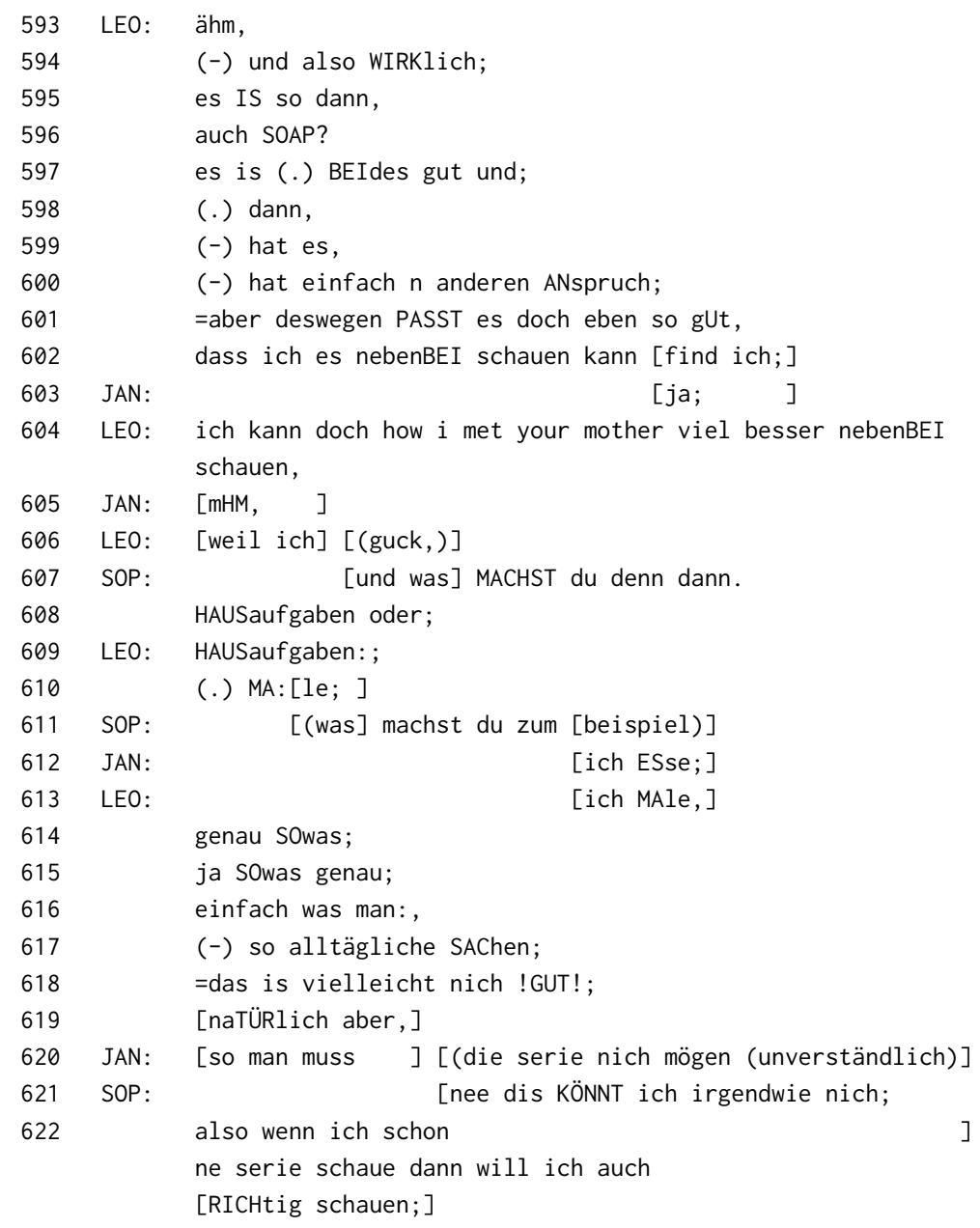


KAT: [ja ich AUCH; ]

624

[es sei denn es is so ne LANGweilige wie how $i$ met your mother; ]

RHG10, 12:58-13:36

Schließlich plädiert er erneut für seine eingangs eingeführte „sowohl-als-auchLösung“ (Beispiel 33, Z. 593-602), die von der anderen Subgruppe jedoch nicht explizit akzeptiert wird. Stattdessen bewerten Sophie und Katharina daraufhin seinen Rezeptionsmodus - nebenbei Hausaufgaben zu erledigen - als negativ und distanzieren sich davon (Z. 622-624). Damit wechseln sie die Fokussierung der Positionierung vom Positionierungsobjekt der Serie hin zur Positionierung von Leon bzw. seiner Rezeption der Serie.

Nach weiteren negativen Bewertungen der Kritiker^innen-Subgruppe (Z. 629640) geht Leon wenig später zum ,Gegenangriff‘ über und etabliert mit TüRKISCH FÜR ANFÄNGER ein neues Positionierungsobjekt, das er mit einer eindeutigen und expliziten Fremdpositionierung verbindet: „, aber ganz ehrlich katharina du hast ja türkisch für ANfänger aufgeschrieben; inwiefern ist DAS, niveauvoller als; “ (Z. 646, 649-650). Damit nimmt er Bezug zu dem Hauptkritikpunkt, How I MET YOUR MOTHER sei billig produziert und wirft Katharina - hier durchaus stellvertretend für die Subgruppe - indirekt eine Bewertung mit zweierlei Maß vor. Das bekräftigt er mit der extreme case-Formulierung, die Serie sei „das niVEAUloseste was ich kenn," - womit er durch die hohe epistemische Gewissheit seiner epistemischen Haltung zugleich genug Autorität zur Beurteilung beansprucht. Hier wäre eine Strukturstelle für die Praktik Absprechen von fremder epistemische Autorität zur Bewertung einer Serie bei K-, Abschnitt 7.1.3) möglich, allerdings spricht ihm niemand die Expertise ab. Stattdessen bedient Katharina seinen Zugzwang: Mit dem Verweis auf unterschiedliche Produktionsorte und damit verbundene kulturelle Vorstellungen von Humor formuliert sie eine subjektiv begründete Erklärung für ihre positive Bewertung der Serie TÜRKISCH FÜR ANFÄNGER (Z. 658-665):

(34) ist für mich der größte SCHROTT auf erden;

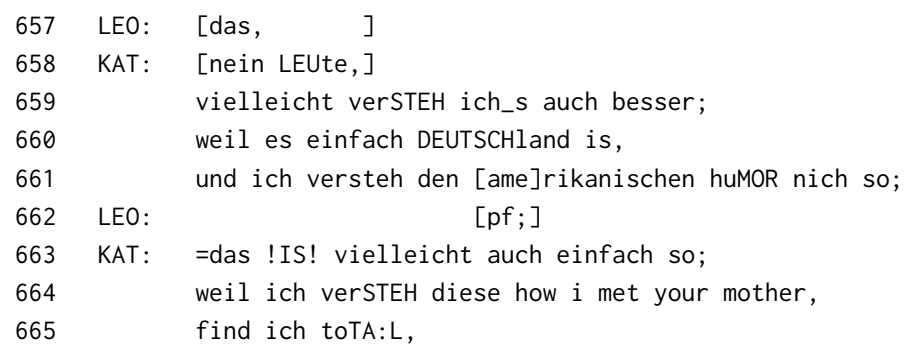




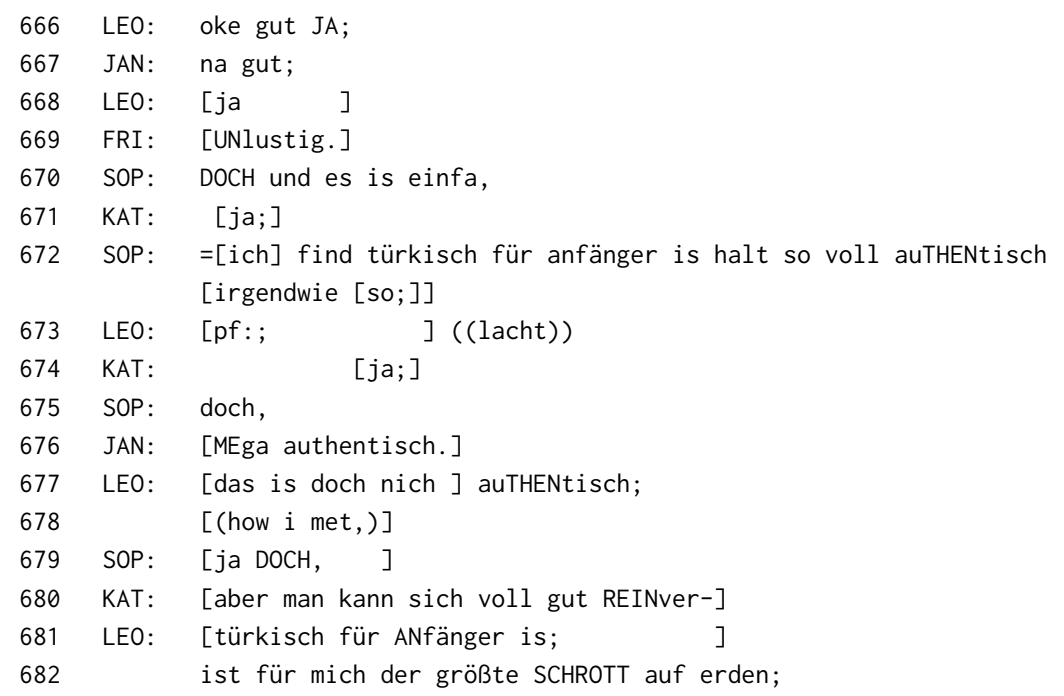

Obwohl Leon und Jan Katharinas Aussage zunächst ratifizieren (Z. 666, 667), setzt sich die Dissens-Sequenz mit der Aushandlung der Frage, inwiefern die Serie authentisch ist (Z. 672-682) und unterschiedlicher Bewertung des zugehörigen Kinofilms (Z. 689-709) solange fort, bis Jan das Thema wechselt (Z. 710). Der Dissens wird also trotz minimaler Zugeständnisse von beiden Seiten nicht aufgelöst, sondern aufrechterhalten. Insgesamt scheint hier keine Präferenz für Konsens erkennbar, da die Teilnehmenden durchaus Vergnügen am Argumentieren, den Bewertungen und dem Schlagabtausch signalisieren. Hilfreich dafür sind hier vermutlich die Hinweise auf den unterschiedlichen „geSCHMACK“ der Beteiligten (Z. 641, 642), der auch in anderen Daten oft als Lösung für kontrastive Bewertungen hinzugezogen wird. Potentiell heikel wird die Interaktionssituation erst, als sich die Beteiligten nicht mehr in Bezug auf die Serie oder ihre eigene Rezeption der Serie positionieren, sondern sich vom Rezeptionsverhalten anderer Teilnehmender abgrenzen.

Insgesamt erscheinen für das - konsensuelle - Aufrechterhalten des Dissens und damit Schärfen der jeweiligen Positionierungen die folgenden Aspekte zentral: Zum einen ist der Auslöser für die Dissenssequenz die unterschiedliche Ausgestaltung der Zugehörigkeit der Serie zu einer Genre-Kategorie (vgl. ähnlich auch in Abschnitt 9.1.4), aus denen sie unterschiedliche Wertungen ableiten. Zum anderen erfolgt das Aufrechterhalten des Dissens darüber, dass erstens nicht alle die gleichen Maßstäbe zum Kontrastieren anlegen, zweitens Zugeständnisse nicht 
von beiden Seiten gleichermaßen angeboten und akzeptiert werden und drittens einer der Teilnehmenden an die Stelle des Positionierungsobjekts rückt (vgl. auch Abschnitt 3.2.4.3)

\subsubsection{Zusammenfassung: Gruppendynamische Positionierungsprozesse zur Herstellung von Gemeinschaft und Distinktion}

In diesem Abschnitt wurden drei Fallstudien vorgestellt, anhand derer sich gruppendynamische Prozesse der Positionierung zur Vergemeinschaftung und Distinktion herausarbeiten lassen. In Abschnitt 7.3.1 wurde rekonstruiert, wie sich die Gruppe darüber vergemeinschaftet, dass sie nicht nur die Serie und detaillierte Wissenselemente der Serie als allen gleichermaßen bekannt und epistemisch zugänglich voraussetzen, sondern ebenfalls ihr subjektives Rezeptionserleben. Das lässt sich an den dynamischen und immer weiter hochgestuften, gestisch und körperlich realisierten Zweitbewertungen erkennen. Auch aus der Analyse in Abschnitt 7.3.2 geht hervor, dass sich die Gruppe nicht nur in ihrer Bewertung, sondern auch in ihrem Wissensstand aneinander anpassen und orientieren kann. Ausschlaggebend ist dafür die Person, die sich als diejenige mit der höchsten epistemischen Autorität zur Bewertung positioniert (vgl. dazu Abschnitt 7.1). Die epistemische Haltung kann mutmaßlich deswegen angepasst werden, weil sich die Bedingungen der Linearität von Fernsehen - in dem Sinne, dass die Rezeption durch die Programmstruktur vorgegeben wird (vgl. Abschnitt 2.1.2) - ändern. Diese Veränderungen der Zeitlichkeit von Serienrezeption können sich also auf die Interaktion und die sprachliche Realisierung von Äußerungen in Bezug auf Tempus- und Moduswechsel auswirken.

Üblicherweise bieten jedoch unterschiedliche Bewertungen einer Serie eine Ausgangsbasis für Distinktionsaktivitäten. So konnte in Abschnitt 7.3.3 gezeigt werden, dass sich so über einen längeren Zeitraum hinweg zwei Subgruppen als Allianzen bilden können, die (vergnüglichen) Dissens aufrechterhalten, indem sie wechselseitige Zugeständnisse ignorieren. Förderlich dafür ist es zum einen, dass sie die Zugehörigkeit der Serie zu einem Genre unterschiedlich ausgestalten und mit entsprechend unterschiedliche Wertmaßstäbe anlegen und zum anderen, dass sie das Positionierungsobjekt von einer Serie zum Rezeptionsverhalten bzw. einem Teilnehmer direkt verschieben (wie es auch Kiesling 2016 als Möglichkeit der Ausdifferenzierung des Objekts beschreibt; vgl. Abschnitt 3.2.4.3). ${ }^{14}$

14 Eine ähnliche Fremdpositionierung, in denen statt einer Serie oder serieller Elemente andere Teilnehmende positioniert werden, findet sich beispielsweise auch in MNG13, Z. 626-642, vgl. Abschnitt 8.1.4. 
Insgesamt zeigt die Analyse gruppenbezogener Positionierungsprozesse, dass zwar meistens Konsens und Vergemeinschaftung angestrebt werden (Abschnitt 6.1.2), dass sich die Präferenzstruktur aber auch umkehren kann und Distinktion (Abschnitt 6.2) präferiert ist. Dann ändert sich die Dynamik von „doing togetherness“ zu „doing being extraordinary“ (vgl. Abschnitt 3.2.3), indem die Teilnehmenden ihren Geschmack kommunizieren und ihn in Relation zu dem der anderen Interagierenden setzen. Über Geschmack wiederum können sie implizit spezifische Identitätsfacetten von sich preisgeben, z.B. in Form von Involvement in fiktionale Formate durch Identifikation mit Figuren (Beispiel 30 oder 34) oder die Abgrenzung von ,billig produzierten“ Formaten (Beispiel 32; vgl. dazu auch Abschnitt 2.1.3) oder zu komplexen Handlungssträngen (Beispiel 31).

\subsection{Zusammenfassung: Positionieren mit lokaler Reichweite - Formen und Funktionen epistemischer und evaluativer Positionierungspraktiken}

In diesem Kapitel wurden Praktiken beschrieben, mit denen sich die Teilnehmenden in Bezug auf konkrete Serien epistemisch und evaluativ positionieren, d.h. die rekonstruierten kommunikativen Aufgaben bearbeiten. Für das epistemische Recht auf Evaluation ist es notwendig, dass über die epistemische Haltung ein ausreichender epistemischer Status kommuniziert wird. Daher wurden die Praktiken analytisch unterteilt in diejenigen, die verwendet werden, um den epistemischen Status aus einer $\mathrm{K}^{+}$-Position heraus hochzustufen (Abschnitt 7.1) und in diejenigen, die den epistemischen Status aus einer $K^{-}$- Position heraus hochstufen (Abschnitt 7.2). Die Praktiken, die Personen mit einer $\mathrm{K}^{+}$-Position einsetzen, zielen darauf ab, epistemische Autorität zum Bewerten einer Serie zu beanspruchen bzw. aufrechtzuerhalten. Das gelingt, indem sie ihrer epistemischen Haltung mehr Gewissheit und/oder mehr Glaubwürdigkeit verleihen. Für die Wahl der Praktik erscheint der interaktive Kontext ausschlaggebend: Je nachdem, wie sich die anderen Teilnehmenden evaluativ und epistemisch positionieren, kann es erforderlich sein, verschiedene Wissensbestände zu managen (Abschnitt 7.1.1) bzw. serienexterne Wissensquellen einzubeziehen (Abschnitt 7.1.2). Bei abweichenden, widersprechenden und/oder heruntergestuften Bewertungen der anderen Teilnehmenden kann zudem die fremde epistemische Autorität abgesprochen (Abschnitt 7.1.3) bzw. ein Rezeptionsmodus empfohlen (Abschnitt 7.1.4) werden. Darüber können sie sich als Expert`innen positionieren.

Andersherum können diejenigen Sprecher*innen, die eine $K^{-}$-Status kommunizieren, Praktiken anwenden, mit denen sie stärker in das Gespräch eingebunden 
sind, indem sie ihre epistemische Haltung mit mehr Gewissheit und/oder Glaubwürdigkeit ausstatten. Dafür können sie partielles Wissen anzeigen (Abschnitt 7.2.1), sich auf die Bewertung anderer berufen (Abschnitt 7.2.2), einen Themenwechsel versuchen zu initiieren (Abschnitt 7.2.3) oder ihren epistemischen Status nachträglich revidieren (Abschnitt 7.2.4). Die Praktiken können dabei auch als gebündelt als Praktikengefüge auftreten. Primäres Ziel für die Praktiken ist es, am Gespräch über den diskursiven Gegenstand zu partizipieren und zu signalisieren, über einen ausreichenden epistemischen Zugang dafür zu verfügen. Vergemeinschaftung und Distinktion innerhalb der Gruppe oder einer Subgruppe sind ebenfalls Ziele, die über die Praktiken erreicht werden.

Um diesbezügliche Prozesse in den Blick zu nehmen, wurden in Abschnitt 7.3 gruppenbezogene Positionierungsprozesse analysiert. Dabei zeigt sich, dass sich die Teilnehmenden an der Person mit der höchsten epistemischen Autorität ausrichten und so sukzessive ihre epistemischen und evaluativen Positionierungen aneinander angleichen können (Abschnitt 7.3.2). Neben dem Vergnügen an gemeinsamen Erinnerungs- und Inszenierungsaktivitäten, für die insbesondere das wechselseitige Vergewissern geteilten Rezeptionserlebens kennzeichnend ist (Abschnitt 7.3.1), kann sich bisweilen die Präferenzstruktur für Konsens umkehren, indem die Teilnehmenden Vergnügen am Aufrechterhalten von Dissens zeigen (Abschnitt 7.3.3). Über Positionierungspraktiken können die Teilnehmenden sowohl Expertise als auch ihren Geschmack kommunizieren und darüber - zum Teil sehr vorsichtig und face-schonend - Identitätsfacetten kommunizieren. Insgesamt fungieren Serien als Verstärker für Positionierungen: Indem die Teilnehmenden eine Serie positionieren, positionieren sie auch zugleich sich selbst in Relation sowohl zu der Serie als auch zu der Gruppe. 